\title{
ANÁLISE DE VARIÂNCIA EM SÉRIES TEMPORAIS
}

\author{
Chang Chiann
}

\author{
DISSERTAÇÃO APRESENTADA \\ $\mathrm{AO}$ \\ INSTITUTO DE MATEMÁTICA E ESTATÍSTICA \\ DA UNIVERSIDADE DE SÃO PAULO \\ PARA OBTENÇÃO DO GRAU DE MESTRE \\ EM \\ ESTATÍSTICA
}

Área de Concentração: Estatística
Orientadora: Profa. Dra. Clélia Maria de Castro Toloi Este trabalho foi financiado pela CAPES

- São Paulo, Junho de 1993 - 
A

meu tio Shao Hua

e

minha tia Xiao Xiao 


\section{Agradecimentos}

Queremos agradecer a todos que contribuíram para a realização deste trabalho, em particular:

- à Professora Doutora Clélia Maria de Castro Toloi, orientadora e amiga, pelo carinho, paciência e dedicação com que transmitiu seus conhecimentos, como também por toda a contribuição que tem dado à minha formação, desde os primeiros cursos de graduação;

- ao Professor Doutor Pedro Alberto Morettin, pela sua orientação constante e pelas sugestões e críticas apresentadas;

- à Professora Lisbeth K. Cordani, pela colaboração;

- a Ana Amélia e Marielza, pelo fornecimento de dados;

- a Cao Ji Kan, pela ajuda na parte de montagem de gráficos;

- a Ting Hua Ching e Sergio Mikio Koyama, pela amizade e incentivo constante. 


\section{Summary}

In this work the analysis of variance is employed to study the variation present in data having the character that the basic responses measured are time series. Our object consists of aplication of the conventional techniques to appropriate transformations of the data.

Basically, there are two kinds of transformation often used: Fourier transform and Walsh-Fourier transform, depending on the data that we would like to analyse.

Some basic models used in the analysis of variance via the Fourier transform of the given data are presented, when the basic responses measured are stationary time series. The models covered in the paper include: case of a common signal, one-way classification, designed experiment, multiple regression and multivariate regression. The cases of both fixed and random effects are considered.

The analysis of variance using Walsh-Fourier is introduced when the basic data is discrete valued and categorical-valued time series, with a limited number of levels (for example, in square waveforms). We also present a brief discussion and comparison of Fourier based and Walsh-Fourier based analysis; we offer some preliminary results on scaling techniques for categorical time series based on the Walsh-Fourier transform.

Finally, we consider two real series (electroscilogram of forty normal mouses and sleep state of sixteen individuals) to illustrate the theoretical results considered in the work. 


\section{Resumo}

Neste trabalho utilizamos a técnica da análise de variância para estudar as variações apresentadas nos dados cujas medidas respostas são séries temporais. O nosso objetivo consiste na aplicação das técnicas convencionais a transformações apropriadas dos dados.

Basicamente, serão utilizados dois tipos de transformação: a de Fourier e a de Walsh-Fourier, dependendo dos dados que queremos analisar.

Alguns modelos usados na análise de variância dos componentes das transformadas de Fourier são apresentados, quando as medidas respostas são séries temporais estacionárias. Os modelos introduzidos incluem: o modelo com um sinal comum, com um fator, o modelo de planejamento, de regressão múltipla e de regressão multivariada. Serão considerados efeitos fixos e aleatórios.

A análise de variância usando Walsh-Fourier é introduzida quando os dados são discretos ou categóricos, com um número limitado de níveis (por exemplo, na forma de "ondas quadradas"). Também fazemos as comparações entre as análises de Fourier e de Walsh-Fourier; abordamos alguns resultados preliminares utilizando o método "scaling" para séries temporais categóricas.

Finalmente, com o objetivo de ilustrar os resultados teóricos apresentados neste trabalho, fazemos a análise de dois conjuntos de dados reais (eletroscilograma de quarenta ratos normais e estado de sono de dezesseis indivíduos). 


\section{Índice}

Capítulo 1 - Introdução 01

Capítulo 2 - Transformada de Fourier e Periodograma .......... 03

2.1 - Algumas definições ................................................. 03

2.2 - Transformada finita de Fourier e Periodograma .. 04

Capítulo 3 - Transformada de Walsh e Periodograma de Walsh

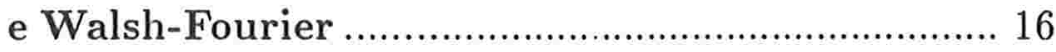

3.1 - Algumas definições ............................................... 16

3.2 - Transformada finita de Walsh-Fourier e Periodograma

Capítulo 4 - Análise de variância usando a Transformada de Fourier 28

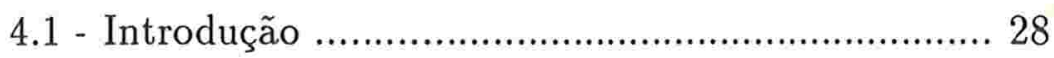

4.2 - Modelo com um sinal comum .............................. 30

4.2.1 - O modelo com sinal determinístico ........... 30

4.2.2 - O modelo com sinal estocástico ………… 34

4.3 - Modelo com um fator …………………………..... 37

4.3.1 - O modelo com fator fixo ……………….... 37

4.3.2 - O modelo com fator aleatório ................... 42

4.4 - Modelo linear geral com matriz de planejamento fixa (modelo de planejamento) ...................................... 45

4.4.1 - O modelo com efeito fixo ......................... 45

4.4.2 - O modelo com efeito aleatório ................... 53 
4.5 - Modelo linear geral

4.6 - Modelo linear multivariado (modelo de regressão multivariado)

Capítulo 5 - Análise de variância usando a Transformada de Walsh-Fourier 66

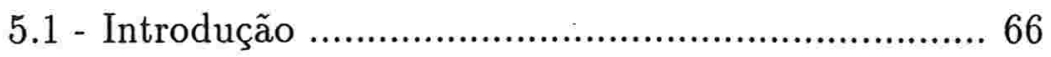

5.2 - Análise no domínio de sequências ......................6 68

5.3 - Comparação entre as análises de Fourier e WalshFourier 72

5.4 - Análise de variância usando Walsh-Fourier 75

5.5 - Séries temporais categóricas 81

Capítulo 6 - Aplicações 86

6.1 - Análise de variância utilizando a Transformada de Fourier 86

6.2 - Análise de variância usando a Transformada de WalshFourier 108

6.3 - Comparação entre a análise de Walsh-Fourier e a de Fourier

Referências Bibliográficas 


\section{Capítulo 1}

Introdução

A motivação desse trabalho se deve ao fato de que, muitas vezes, queremos utilizar uma análise de variância para estudar as variações apresentadas nos dados cujas medidas respostas são séries temporais. A análise convencional não é adequada neste caso, uma vez que nossos dados são frequentemente correlacionados, isto é, dependentes no tempo. O nosso objetivo é "adaptar" os dados, no sentido de amenizar a dependência entre eles e apresentar soluções para resolver nosso problema. Veremos que, uma vez feita a transformação dos dados, a análise utilizada será uma aplicação das técnicas convencionais de análise de variância.

Basicamente, serão utilizados dois tipos de transformação: a de Fourier e a de Walsh-Fourier, dependendo do tipo de dados que queremos analisar.

A análise de Fourier, que se utiliza do sistema ortogonal das funções senoidais, é provavelmente uma das ferramentas mais importantes para lidar com as correlações presentes em séries temporais estacionárias. Tal análise transforma a série para o domínio de frequências, onde os pontos adjacentes são assintoticamente independentes. A transformação introduzida é a de Fourier e as observações transformadas além de serem assintoticamente não correlacionadas também terão uma distribuição aproximadamente normal.

A análise de Walsh, baseada no sistema ortogonal das funções de Walsh, definidas através das funções de Rademacher, é ideal para analisar os processos que contêm dados discretos ou categóricos, com um número limitado de níveis (por exemplo, na forma de "ondas quadradas"). Tal análise transforma a série para o domínio de sequências, onde os pontos vizinhos são assintoticamente independentes. A transformação introduzida é a de Walsh-Fourier e tem sido usada para vários propósitos, inicialmente na área de engenharia, tal como identificação de palavras, codificação e transmissão de imagens; 
posteriormente na área biológica e médica, principalmente na descrição de ciclos nos sinais de eletroencefalograma(EEG). Todas as idéias da análise de Fourier são colocadas na análise de Walsh. Tal como na transformada de Fourier, as transformadas de Walsh são assintoticamente independentes com distribuição aproximadamente normal.

No Capítulo 2, baseado em Brillinger(1981), abordamos alguns conceitos fundamentais sobre a transformada de Fourier e periodograma, bem como apresentamos estimadores assintoticamente não viciados da função espectral de uma série temporal.

No Capítulo 3, utilizando Morettin(1972), introduzimos as funções de Walsh e os elementos da análise de Walsh e Walsh-Fourier em alguns detalhes, bem como definimos a transformada de Walsh, o espectro de Walsh-Fourier e alguns resultados assintóticos para a transformada de Walsh-Fourier.

Alguns modelos usados na análise de variância dos componentes das transformadas de Fourier são apresentados no Capítulo 4, incluindo o modelo com um sinal comum, com um fator, o modelo de planejamento, de regressão múltipla e de regressão multivariada. Serão considerados efeitos fixo e aleatório.

No Capítulo 5, apresentamos as análises no domínio de sequências e as comparações entre as análises de Fourier e de Walsh-Fourier. A seguir, uma análise de variância usando Walsh-Fourier é introduzida. Também abordamos alguns resultados preliminares utilizando o método "scaling" para séries temporais categóricas, baseado na transformada de Walsh-Fourier.

Finalmente, no Capítulo 6, fazemos a análise de dois conjuntos de dados reais, utilizando os resultados teóricos obtidos nos capítulos anteriores. Os programas especialmente desenvolvidos para tais análises se encontram no Apêndice. 


\section{Capítulo 2}

\section{Transformada de Fourier e Periodograma}

\subsection{Algumas Definições}

Definição 2.1: Um processo estocástico $X=\{X(t, \omega), t \in T, \omega \in \Omega\}$ é uma família de variáveis aleatórias definidas num espaço de probabilidade $(\omega, \Lambda, P)$ e indexadas pelos elementos de um conjunto de parâmetros T.

$\mathrm{Na}$ realidade, um processo estocástico é uma função de dois argumentos, $X(t, \omega), t \in T, \omega \in \Omega$. Para cada $t \in T$, a função $X(t, \cdot)$ é mensurável relativamente a $\Lambda$. Por outro lado, para cada $\omega \in \Omega$, fixado, obteremos uma função $X(\cdot, \omega)$ de t: $T \mapsto \Re$, que é chamada trajetória, realização, função amostral do processo ou série temporal.

Para facilitar a notação, representaremos $X(t, \omega)$ por $X(t)$.

Definição 2.2: Dado um processo estocástico $\{X(t), t \in T\}$. Definimos suas distribuições finito-dimensionais por

$$
F\left(x_{1}, \cdots, x_{n} ; t_{1}, \cdots, t_{n}\right)=\operatorname{Prob}\left(X\left(t_{1}\right) \leq x_{1}, \cdots, X\left(t_{n}\right) \leq x_{n}\right),
$$

para todo $n \geq 1$ e $t_{1}, \cdots, t_{n} \in T$.

Definição 2.3: As funções média e covariância de um processo $X(t)$ são dadas por

$$
\begin{aligned}
& E X(t)=\int_{-\infty}^{\infty} x d F(x ; t)=\mu_{x}(t) \\
& \operatorname{Cov}\left[X\left(t_{1}\right), X\left(t_{2}\right)\right]=\iint\left[x_{1}-\mu_{x}\left(t_{1}\right)\right]\left[x_{2}-\mu_{x}\left(t_{2}\right)\right] d F\left[x_{1}, x_{2} ; t_{1}, t_{2}\right] \\
&=E\left[X\left(t_{1}\right) X\left(t_{2}\right)\right]-E\left[X\left(t_{1}\right)\right] E\left[X\left(t_{2}\right)\right] \\
&=c_{x x}\left(t_{1}, t_{2}\right) .
\end{aligned}
$$


Em particular, se $t_{1}=t_{2}=t$, temos a variância do processo

$$
\operatorname{Var} X(t)=\int\left[x-\mu_{x}(t)\right]^{2} d F(x ; t)=E\left[X^{2}(t)\right]-E^{2}[X(t)]=c_{x x}(t, t) .
$$

Definição 2.4: Um processo estocástico $\{X(t), t \in T\}$ é estritamente estacionário se todas as distribuições finito-dimensionais (2.1) permanecem as mesmas sob uma translação do tempo, ou seja,

$$
F\left(x_{1}, \cdots, x_{n} ; t_{1}+\tau, \cdots, t_{n}+\tau\right)=F\left(x_{1}, \cdots, x_{n} ; t_{1}, \cdots, t_{n}\right),
$$

para quaisquer $t_{1}, \cdots, t_{n} ; \tau \in T$.

Definição 2.5: Um processo estocástico $\{X(t), t \in T\}$ é estacionário de segunda ordem, ou fracamente estacionário, ou ainda estacionário em sentido amplo se

(i) $E[X(t)]=\mu_{x}$, constante, $\forall t \in T$.

(ii) $E\left[X^{2}(t)\right]<\infty, \forall t \in T$.

(iii) $c_{x x}\left(t_{1}, t_{2}\right)=c_{x x}\left(t_{1}-t_{2}\right)$ só depende da defasagem $\left|t_{1}-t_{2}\right|, \forall t_{1}, t_{2} \in T$.

Definição 2.6: (a) Seja $\left(X_{1}, \cdots, X_{r}\right)$ uma variável aleatória r-dimensional com $E\left|X_{j}\right|^{r}<\infty, j=1, \cdots, r$, onde $X_{j}$ são reais ou complexas. $O$ cumulante conjunto de ordem $r$ é dado por

$$
\operatorname{Cum}\left(X_{1}, \cdots, X_{r}\right)=\sum_{\nu}(-1)^{p-1}(p-1) !\left(E \prod_{j \in \nu_{1}} X_{j}\right) \cdots\left(E \prod_{j \in \nu p} X_{j}\right),
$$

onde a soma é sobre todas as partições $\nu=\left(\nu_{1}, \cdots, \nu_{p}\right)$ dos inteiros $(1, \cdots, r)$.

zendo $E|X(t)|^{k}<\infty$, então

(b) Seja uma série temporal $\{X(t), t=0, \pm 1, \cdots\}$ satisfa-

$$
C_{x}\left(t_{1}, \cdots, t_{r}\right)=\operatorname{Cum}\left(X_{t_{1}}, \cdots, X_{t_{r}}\right)
$$

é denominada função cumulante conjunta de ordem $\mathrm{r}$ da série $X(t)$. 
Resultado: O Cum $\left(X_{1}, \cdots, X_{r}\right)$ é dado pelo coeficiente de

$$
i^{r} t_{1} \cdots t_{r}
$$

na série de expansão de Taylor de $\log \left(E \exp \left(i \sum_{j=1}^{r} X_{j} t_{j}\right)\right)$.

Propriedades:

(i) $\operatorname{Cum}\left(a_{1} X_{1}, \cdots, a_{r} X_{r}\right)=a_{1} \cdots a_{r} \operatorname{Cum}\left(X_{1}, \cdots, X_{r}\right)$, para $a_{1}, \cdots, a_{r}$ constantes.

(ii) $\operatorname{Cum}\left(X_{1}, \cdots, X_{r}\right)$ é simétrica em seus argumentos.

(iii) $\operatorname{Cum}\left(X_{j}\right)=E X_{j}$, para $j=1, \cdots, r$.

(iv) $\operatorname{Cum}\left(X_{j}, \overline{X_{j}}\right)=\operatorname{Var} X_{j}$ e $\operatorname{Cum}\left(X_{j}, \overline{X_{k}}\right)=\operatorname{Cov}\left(X_{j}, X_{k}\right)$, para $j, k=$ $1, \cdots, r$.

(v) Se algum subconjunto dos $\left(X_{1}, \cdots, X_{r}\right)$ é independente dos demais, então $\operatorname{Cum}\left(X_{1}, \cdots, X_{r}\right)=0$.

(vi) Para variável aleatória $\left(Z_{1}, X_{1}, \cdots, X_{r}\right)$,

$$
\operatorname{Cum}\left(X_{1}+Z_{1}, X_{2}, \cdots, X_{r}\right)=\operatorname{Cum}\left(X_{1}, \cdots, X_{r}\right)+\operatorname{Cum}\left(Z_{1}, X_{2}, \cdots, X_{r}\right) .
$$

(vii) Para $u$ constante e $r=2,3, \cdots$,

$$
\operatorname{Cum}\left(X_{1}+u, X_{2}, \cdots, X_{r}\right)=\operatorname{Cum}\left(X_{1}, X_{2}, \cdots, X_{r}\right) .
$$

(viii) Se variável aleatória $\left(X_{1}, \cdots, X_{r}\right)$ e $\left(Z_{1}, \cdots, Z_{r}\right)$ são independentes, então

$$
\operatorname{Cum}\left(X_{1}+Z_{1}, \cdots, X_{r}+Z_{r}\right)=\operatorname{Cum}\left(X_{1}, \cdots, X_{r}\right)+\operatorname{Cum}\left(Z_{1}, \cdots, Z_{r}\right) .
$$

Definição 2.7: Suponha que a série temporal $X(t), t=0, \pm 1, \cdots$, com função de autocovariância (f.a.c) $\operatorname{Cov}(X(t+u), X(t))=c_{x x}(u), t, u=$ $0, \pm 1, \cdots$ satisfazendo

$$
\sum_{u=-\infty}^{\infty}\left|c_{x x}(u)\right|<\infty
$$


então o espectro de $X(t)$, na frequência $\lambda$, é dado por

$$
f_{x x}(\lambda)=(2 \pi)^{-1} \sum_{u=-\infty}^{\infty} \exp (-i \lambda u) c_{x x}(u)
$$

para $-\infty<\lambda<\infty$.

Sob o ponto de vista da quantidade de informação probabilística que fornecem, o espectro e a função de autocovariância(f.a.c) são ferramentas equivalentes.

Demonstra-se que o espectro $f_{x x}(\lambda)$ definido por (2.9) é limitado, nãonegativo e uniformemente contínuo. Além disso, $f_{x x}(\lambda)$ é par e periódico de período $2 \pi$.

Como $f_{x x}(\lambda)$ é par e periódico de período $2 \pi$, basta tomar $\lambda \in[-\pi, \pi]$ e utilizar o intervalo $[0, \pi]$ como o domínio fundamental da definição do espectro $f_{x x}(\lambda)$.

A relação (2.9) pode ser invertida e a função de autocovariância $c_{x x}(u)$ pode ser escrita como

$$
c_{x x}(u)=\int_{-\pi}^{\pi} \exp (i \alpha u) f_{x x}(\alpha) d \alpha
$$

para $u=0, \pm 1, \cdots$. No caso particular $u=0$, temos

$$
\operatorname{Var} X(t)=\int_{-\pi}^{\pi} f_{x x}(\alpha) d \alpha
$$

Definição 2.8: (a) Um vetor aleatório real r-dimensional $X=\left(X_{1}, \cdots, X_{r}\right)^{\prime}$ tem uma distribuição conjunta normal multivariada de dimensão $\mathrm{r}$, com média $\mu_{x}=\left[\mu_{1}, \cdots, \mu_{r}\right]^{\prime}$ e matriz de covariância $\Sigma_{x x}$, se sua função densidade é

$$
f\left(x_{1}, \cdots, x_{r}\right)=(2 \pi)^{-r / 2}\left|\Sigma_{x x}\right|^{-1} e^{-\frac{1}{2}\left(X-\mu_{x}\right)^{\prime} \Sigma_{x x}^{-1}\left(X-\mu_{x}\right)} .
$$

Notação: $X: N_{r}\left(\mu_{x}, \Sigma_{x x}\right)$. 
(b) Se X é um vetor r-dimensional, com componentes complexas, dizemos que $\mathrm{X}$ tem uma distribuição normal complexa multivariada de dimensão $\mathrm{r}$, com média $\mu_{x}$ e matriz de covariâncias $\Sigma_{x x}$, se o vetor

$$
\left[\begin{array}{c}
\operatorname{Re} X \\
\operatorname{Im} X
\end{array}\right]
$$

é distribuido como

$$
N_{2 r}\left(\left[\begin{array}{c}
\operatorname{Re} \mu_{x} \\
\operatorname{Im} \mu_{x}
\end{array}\right] ; 1 / 2\left[\begin{array}{cc}
\operatorname{Re} \Sigma_{x x} & -\operatorname{Im} \Sigma_{x x} \\
\operatorname{Im} \Sigma_{x x} & \operatorname{Re} \Sigma_{x x}
\end{array}\right]\right),
$$

onde $\Sigma_{x x}$ é uma matriz Hermitiana não negativa definida de dimensão $r$ x $r$.

Notação: $X: N_{r}^{c}\left(\mu_{x}, \Sigma_{x x}\right)$, com

$$
\begin{gathered}
E(X)=\mu_{x}, \\
E\left(\left(X-\mu_{x}\right)\left(\overline{X-\mu_{x}}\right)^{\prime}\right)=\Sigma_{x x}
\end{gathered}
$$

$\mathrm{e}$

$$
E\left(\left(X-\mu_{x}\right)\left(X-\mu^{\prime} X\right)^{\prime}\right)=0
$$

Se $\Sigma_{x x}$ é não-singular, então a função densı..... ue $X$ é dada por

$$
\begin{aligned}
f\left(x_{1}, \cdots, x_{r}\right)= & \pi^{-r}\left(\left|\Sigma_{x x}\right|\right)^{-1} \exp \left(-\overline{\left(X-\mu_{x}\right)^{\prime}} \Sigma_{x x}^{-1}\left(X-\mu_{x}\right)\right) \\
& * \prod_{j}\left(d \operatorname{Re} X_{j}\right)\left(d \operatorname{Im} X_{j}\right),
\end{aligned}
$$

para $-\infty<\operatorname{Re} X_{j}, \operatorname{Im} X_{j}<\infty$.

PRopRIEDADES:

(i) Se $\Sigma_{x x}$ é uma matriz diagonal, então as componentes de $X$ são estatisticamente independentes.

(ii) No caso que $r=1$, se $X$ tem dis.

$\operatorname{Im} X$ são v.a. independentes com distribuis.....

$$
N_{1}\left(\operatorname{Re} \mu_{x}, \sigma_{x x} / 2\right)
$$

e

$$
N_{1}\left(\operatorname{Im} \mu_{x}, \sigma_{x x} / 2\right)
$$

respectivamente. 
Definição 2.9: (a) Dizemos que a sequência vetorial $\zeta_{T}$ de dimensão $r$, $T=1,2, \cdots$, é assintoticamente $N_{r}\left(\mu_{T}, \Sigma_{T}\right)$ se a sequência $\sum_{T}^{-1 / 2}\left(\zeta_{T}-\mu_{T}\right) \stackrel{\mathcal{D}}{\rightarrow}$ $N_{r}(0, I)$.

(b) Dizemos que a sequência vetorial $\zeta_{T}$ de dimensão $r, T=1,2, \cdots$, é assintoticamente $N_{r}^{c}\left(\mu_{T}, \Sigma_{T}\right)$ se a sequência $\sum_{T}^{-1 / 2}\left(\zeta_{T}-\mu_{T}\right) \stackrel{\mathcal{D}}{\rightarrow} N_{r}^{c}(0, I)$.

Definição 2.10: (a) A matriz de dimensão $r \times r$ das variáveis aleatórias

$$
W=\sum_{j=1}^{n} X_{j} X j^{\prime}
$$

tem uma distribuição Wishart de dimensão $r$ e grau de liberdade $n$ se $X_{1}, \cdots, X_{n}$ são independentes com distribuição normal $N_{r}\left(0, \Sigma_{x x}\right)$.

Notação: $W: W_{r}\left(n, \Sigma_{x x}\right)$.

(b) A matriz de dimensão $r \times r$ das variáveis aleatórias

$$
W=\sum_{j=1}^{n} X_{j} \overline{X_{j}^{\prime}}
$$

tem uma distribuição Wishart complexa de dimensão $r$ e grau de liberdade $n$ se $X_{1}, \cdots, X_{n}$ são independentes com distribuição normal complexa $N_{r}^{c}\left(0, \Sigma_{x x}\right)$.

Notação: $W: W_{r}^{c}\left(n, \Sigma_{x x}\right)$.

\section{PROPRIEDADES:}

(i) $\bar{W}=W^{\prime}$.

(ii) $E W=n \Sigma_{x x}$.

(iii) $\operatorname{Cov}\left(W_{j k}, W_{l m}\right)=E\left(\left(W_{j k}-n \Sigma_{j k}\right) \overline{\left(W_{l m}-n \Sigma_{l m}\right)}\right)=n \Sigma_{j l} \bar{\Sigma}_{k m}$.

(iv) Se W tem distribuição $W_{r}^{c}(n, \Sigma)$, então $W_{j j}$ tem distribuição

$$
\Sigma_{j j} \chi_{2 n}^{2} / 2 \text {. }
$$


(v) $\operatorname{Se} Y: N_{n}^{c}\left(\mu, \sigma^{2} I\right) \mathrm{e}$

$$
\overline{Y^{\prime}} Y=\overline{Y^{\prime}} A_{1} Y+\cdots+\overline{Y^{\prime}} A_{K} Y,
$$

onde $A_{k}$ Hermitiana de posto $n_{k}, k=1, \cdots, K$, então $\overline{Y^{\prime}} A_{1} Y, \ldots, \overline{Y^{\prime}} A_{K} Y$ são independentes e $\overline{Y^{\prime}} A_{k} Y$ tem distribuição (qui-quadrado não central)

se e somente se, $n_{1}+n_{2}+\cdots+n_{K}=n$.

$$
\sigma^{2} \chi_{2 n_{k}}^{2}\left(\overline{\mu^{\prime}} \mu / \sigma^{2}\right) / 2,
$$

\author{
cesomentese, $n_{1}+n_{2}+\cdots+n_{K}=n_{1}$
}

\title{
2.2 Transformada Finita de Fourier e Peri- odograma
}

Seja $X(n), n=0, \pm 1, \cdots$, um processo estacionário real. A transformada finita de Fourier dos valores $X(0), X(1), \cdots, X(T-1)$ de $X(n)$ é dada por

$$
d_{x}^{(T)}(\lambda)=\sum_{t=0}^{T-1} X(t) \exp (-i \lambda t),-\infty<\lambda<\infty .
$$

No caso que $T=2 n+1, n$ inteiro, podemos escrever

$$
d_{x}^{(T)}(\lambda)=\exp (-i \lambda n) \sum_{u=-n}^{n} X(u+n) \exp (-i \lambda u) .
$$

A transformada discreta de Fourier dos valores $X(0), \cdots, X(T-1)$ de $X(n)$ é dada por

$$
d_{x}^{(T)}\left(\frac{2 \pi s}{T}\right), s=0, \pm 1, \cdots, T-1 .
$$

PROPRIEDADES:

(i) $d_{x}^{(T)}(\lambda+2 \pi)=d_{x}^{(T)}(\lambda)$.

(ii) $\overline{d_{x}^{(T)}(\lambda)}=d_{x}^{(T)}(-\lambda)$, se $\mathrm{X}(\mathrm{t})$ é real. 
(iii) $d_{\alpha x+\beta y}^{(T)}(\lambda)=\alpha d_{x}^{(T)}(\lambda)+\beta d_{y}^{(T)}(\lambda)$, se $\alpha, \beta$ são escalares e $X(t), Y(t)$ são processos estacionários não correlacionados.

A relação (2.19) pode ser invertida e a série $X(t)$ pode ser escrita como

$$
X(t)=(2 \pi)^{-1} \int_{0}^{2 \pi} \exp (i t \lambda) d_{x}^{(T)}(\lambda) d \lambda, \quad t=0, \pm 1, \cdots, T-1
$$

ou

$$
X(t)=T^{-1} \sum_{s=0}^{T-1} \exp \left(i \frac{2 \pi s t}{T}\right) d_{x}^{(T)}\left(\frac{2 \pi s}{T}\right), \quad t=0, \cdots, T-1 .
$$

Teorema 2.1: $\quad$ Se $X(t)=\left(X_{1}(t), \cdots, X_{r}(t)\right)^{\prime}$ é estritamente estacionário,

$$
\sum_{\mu_{1}, \cdots, \mu_{k-1}}^{\infty}\left|C_{x}\left(\mu_{1}, \cdots, \mu_{k-1}\right)\right|<\infty
$$

e $s_{j}(T)$ um inteiro com $\lambda_{j}(T)=\frac{2 \pi s_{j}(T)}{T} \rightarrow \lambda_{j}$ quando $T \rightarrow \infty$ para $j=$ $1, \cdots, J$, então

(i) para $2 \lambda_{j}(T), \lambda_{j}(T) \pm \lambda_{k}(T) \not \equiv 0(\bmod 2 \pi), 1 \leq j<k \leq J, T=1,2, \cdots$, temos que $d_{x}^{(T)}\left(\lambda_{j}(T)\right) \stackrel{\mathcal{D}}{\rightarrow} N_{r}^{c}\left(0,2 \pi T f_{x x}\left(\lambda_{j}\right)\right)$ independentes;

(ii) para $\lambda=0, \pm 2 \pi, \cdots, d_{x}^{(T)}(\lambda) \stackrel{\mathcal{D}}{\rightarrow} N_{r}\left(T \mu_{x}, 2 \pi T f_{x x}(\lambda)\right)$ independentes e

(iii) para $\lambda= \pm \pi, \pm 3 \pi, \cdots, d_{x}^{(T)}(\lambda) \stackrel{\mathcal{D}}{\rightarrow} N_{r}\left(0,2 \pi T f_{x x}(\lambda)\right)$ independentes.

Outros teoremas do limite central para séries estacionárias são dados em Rosenblatt (1956, 1961), Leonov and Shiryaev (1960), Iosifescu e Theodorescu (1969) e Philipp (1969).

$O$ periodograma de uma série temporal estacionária $X(t), t=0, \cdots, T-1$ é dado por

$$
I_{x x}^{(T)}(\lambda)=(2 \pi T)^{-1}\left|d_{x}^{(T)}(\lambda)\right|^{2}, \lambda \not \equiv 0(\bmod 2 \pi) .
$$


Segundo o Teorema 2.1, temos que

$$
d_{x}^{(T)}(\lambda) \stackrel{\mathcal{D}}{\rightarrow} \begin{cases}N_{1}^{c}\left(0,2 \pi T f_{x x}(\lambda)\right) & \text { se } \lambda \not \equiv 0(\bmod \pi) \\ N_{1}\left(T \mu_{x}, 2 \pi T f_{x x}(\lambda)\right) & \text { se } \lambda=0, \pm 2 \pi, \cdots \\ N_{1}\left(0,2 \pi T f_{x x}(\lambda)\right) & \text { se } \lambda= \pm \pi, \pm 3 \pi, \cdots\end{cases}
$$

sugerindo que $I_{x x}^{(T)}(\lambda)$ é um possível estimador para $f_{x x}(\lambda)$. Notamos que $I_{x x}^{(T)}(\lambda)$ tem mesmas propriedades do espectro $f_{x x}(\lambda)$.

Teorema 2.2: Se $X(t), t=0, \pm 1, \cdots$, é uma série temporal satisfazendo (2.8), então

$$
\begin{aligned}
E I_{x x}^{(T)}(\lambda)= & (2 \pi T)^{-1} \int_{-\pi}^{\pi}\left[\frac{\operatorname{sen} T(\lambda-\alpha) / 2}{\operatorname{sen}(\lambda-\alpha) / 2}\right]^{2} f_{x x}(\alpha) d \alpha \\
& +(2 \pi T)^{-1}\left[\frac{\operatorname{sen} T \lambda / 2}{\operatorname{sen} \lambda / 2}\right]^{2} \mu_{x}^{2}
\end{aligned}
$$

para $-\infty<\lambda<\infty$. No caso $\lambda \not \equiv 0(\bmod 2 \pi)$, o último termo de $(2.24)$ é pequeno e notamos que $E I_{x x}^{(T)}(\lambda)$ é aproximadamente igual a uma média ponderada do espectro com peso concentrado na vizinhança de $\lambda$. O termo $\left[\frac{\operatorname{sen} T(\lambda-\alpha) / 2}{\operatorname{sen}(\lambda-\alpha) / 2}\right]^{2}$ é denominado núcleo de Fejer.

Corolário 2.1: Sob as condições do Teorema 2.2 , se $\lambda \not \equiv 0(\bmod 2 \pi)$, então $I_{x x}^{(T)}(\lambda)$ é um estimador assintoticamente não viciado de $f_{x x}(\lambda)$, isto é,

$$
\lim _{T \rightarrow \infty} E\left(I_{x x}^{(T)}(\lambda)\right)=f_{x x}(\lambda)
$$

Teorema 2.3: Sob as condições do Teorema 2.2 e se

$$
\sum_{u}\left|u \| c_{x x}(u)\right|<\infty
$$

então

$$
E I_{x x}^{(T)}(\lambda)=f_{x x}(\lambda)+(2 \pi T)^{-1}\left[\frac{\operatorname{sen} T \lambda / 2}{\operatorname{sen} \lambda / 2}\right]^{2} \mu_{x}^{2}+O\left(T^{-1}\right)
$$

O termo $O\left(T^{-1}\right)$ é uniforme em $\lambda$. 
Teorema 2.4: Se $X(t)$ é Gaussiano e $I_{x x}^{(T)}(\lambda)$ é dado por (2.23) para $\lambda$, $\mu \not \equiv 0(\bmod 2 \pi)$, temos que

$$
\begin{aligned}
& \operatorname{Cov}\left(I_{x x}^{(T)}(\lambda), I_{x x}^{(T)}(\mu)\right)= \\
& \quad\left(\left[\frac{\operatorname{sen} T(\lambda+\mu) / 2}{T \operatorname{sen}(\lambda+\mu) / 2}\right]^{2}+\left[\frac{\operatorname{sen} T(\lambda-\mu) / 2}{T \operatorname{sen}(\lambda-\mu) / 2}\right]^{2}\right) f_{x x}(\lambda)+O\left(T^{-1}\right) .
\end{aligned}
$$

Teorema 2.5: Nas mesmas condições do Teorema 2.4, se $\mu=\frac{2 \pi r}{T}$, $\lambda=\frac{2 \pi s}{T}, \operatorname{com} r, s, r \pm s \not \equiv 0(\bmod T)$, inteiros, então

$$
\begin{aligned}
& \operatorname{Var} I_{x x}^{(T)}(\lambda)=f_{x x}(\lambda)^{2}+O\left(T^{-1}\right), \\
& \operatorname{Cov}\left(I_{x x}^{(T)}(\lambda), I_{x x}^{(T)}(\mu)\right)=O\left(T^{-1}\right) .
\end{aligned}
$$

Demonstrações, ver Brillinger(1981).

Teorema 2.6: Se $X(t)$ é estritamente estacionário satisfazendo (2.22) e $s_{j}(T)$ um inteiro com $\lambda_{j}(T)=\frac{2 \pi s_{j}(T)}{T} \rightarrow \lambda_{j}$, quando $T \rightarrow \infty, j=1,2, \cdots, J$, então

(i) $\operatorname{para} 2 \lambda_{j}(T), \lambda_{j}(T) \pm \lambda_{k}(T) \not \equiv 0(\bmod 2 \pi), 1 \leq j<k \leq J$ e $T=1,2, \cdots$, temos que $I_{x x}^{(T)}\left(\lambda_{j}(T)\right) \stackrel{\mathcal{D}}{\rightarrow} f_{x x}\left(\lambda_{j}\right) \chi_{2}^{2} / 2$ independentes;

(ii) para $\lambda= \pm \pi, \pm 3 \pi, \cdots, I_{x x}^{(T)}(\lambda) \stackrel{\mathcal{D}}{\rightarrow} f_{x x}(\lambda) \chi_{1}^{2}$ independentes e

(iii) para $\lambda \equiv 0(\bmod 2 \pi), I_{x x}^{(T)}(\lambda) \stackrel{\mathcal{D}}{\rightarrow}\left\{\begin{array}{ll}f_{x x}(\lambda) \chi_{1}^{2} & \text { se } \mu_{x}=0 \\ f_{x x}(\lambda) \chi_{1}^{2}(\psi) & \text { se } \mu_{x} \neq 0,\end{array}\right.$ onde $\psi=\left|\mu_{x}\right| \sqrt{T /\left(2 \pi f_{x x}(\lambda)\right)}$ é o parâmetro de não-centralidade do quiquadrado.

Podemos notar que, embora o periodograma seja assintoticamente não viciado, ele não é consistente (Brillinger, 1981).

Devido a grande instabilidade do periodograma (estimador não consistente do espectro), o Teorema 2.6 sugere construir estimadores espectrais que têm propriedades melhores que o periodograma. 
De acordo com o Teorema 2.6, se $s(T)$, inteiro com $\frac{2 \pi s(T)}{T} \rightarrow \lambda$ e $2[s(T)+$ $j] \not \equiv 0(\bmod T)$, então $I_{x x}^{(T)}\left(\frac{2 \pi[s(T)+j]}{T}\right), j=0, \pm 1, \cdots, \pm m$, são aproximadamente independentes com distribuição assintótica $f_{x x}(\lambda) \chi_{2}^{2} / 2$. Assim, um novo estimador para $f_{x x}(\lambda)$, denominado periodograma suavizado, é dado por

$$
f_{x x}^{(T)}(\lambda)= \begin{cases}(2 m+1)^{-1} \sum_{j=-m}^{m} I_{x x}^{(T)}\left(\frac{2 \pi[s(T)+j]}{T}\right) & \text { se } \lambda \not \equiv 0(\bmod \pi) \\ (2 m)^{-1}\left(\sum_{j=-m}^{-1}+\sum_{j=1}^{m}\right) I_{x x}^{(T)}\left(\lambda+\frac{2 \pi j}{T}\right) & \text { se } \lambda=0(\bmod 2 \pi) \\ & \text { ou } \lambda= \pm \pi, \pm 3 \pi, \cdots \\ m^{-1} \sum_{j=1}^{m} I_{x x}^{(T)}\left(\lambda-\frac{\pi}{T}+\frac{2 \pi j}{T}\right) & \text { e } T \text { é par } \\ & \text { se } \lambda= \pm \pi, \pm 3 \pi, \cdots \\ & \text { e } T \text { é impar }\end{cases}
$$

que é uma média das ordenadas do periodograma em torno de $\lambda$.

Definindo

$$
F_{T}(\lambda)=(2 \pi T)^{-1}\left[\frac{\operatorname{sen} T \lambda / 2}{\operatorname{sen} \lambda / 2}\right]^{2}
$$

que é núcleo de Fejer,

$$
\begin{gathered}
A_{T}^{m}(\lambda)=(2 m+1)^{-1} \sum_{j=-m}^{m} F_{T}\left(\lambda-\frac{2 \pi j}{T}\right), \\
B_{T}^{m}(\lambda)=(2 m)^{-1}\left(\sum_{j=-m}^{-1}+\sum_{j=1}^{m}\right) F_{T}\left(\lambda-\frac{2 \pi j}{T}\right)
\end{gathered}
$$

e

$$
C_{T}^{m}(\lambda)=(2 m)^{-1} \sum_{j=1}^{m}\left(F_{T}\left(\lambda-\frac{\pi}{T}+\frac{2 \pi j}{T}\right)+F_{T}\left(\lambda+\frac{\pi}{T}-\frac{2 \pi j}{T}\right)\right),
$$

para $-\infty<\lambda<\infty$, podemos notar que $A_{T}^{m}, B_{T}^{m}$ e $C_{T}^{m}$ são não-negativos, têm integral um sobre intervalo $[-\pi, \pi]$ com periodicidade de $2 \pi$. 
Teorema 2.7: Se $X(t), t=0, \pm 1, \cdots$, e $\sum_{u=-\infty}^{\infty}\left|c_{x x}(u)\right|<\infty$, então

$$
E f_{x x}^{(T)}(\lambda)= \begin{cases}\int_{-\pi}^{\pi} A_{T}^{m}(\alpha) f_{x x}\left(\frac{2 \pi s(T)}{T}-\alpha\right) d \alpha & \text { se } \lambda \not \equiv 0(\bmod 2 \pi) \\ \int_{-\pi}^{\pi} B_{T}^{m}(\alpha) f_{x x}(\lambda-\alpha) d \alpha & \text { se } \lambda \equiv 0(\bmod 2 \pi) \\ & \text { or } \lambda= \pm \pi, \pm 3 \pi, \cdots \\ \int_{-\pi}^{\pi} C_{T}^{m}(\alpha) f_{x x}(\lambda-\alpha) d \alpha & \text { e T é par } \\ & \text { se } \lambda= \pm \pi, \pm 3 \pi, \cdots \\ & \text { e T é impar. }\end{cases}
$$

Corolário 2.2: $\quad$ Se $\lambda-\frac{2 \pi s(T)}{T} \rightarrow O\left(T^{-1}\right), m$ é constante, $m \ll T$ e (2.25) valido, então

$$
E f_{x x}^{(T)}(\lambda)=f_{x x}(\lambda)+O\left(T^{-1}\right)
$$

para $-\infty<\lambda<\infty$. Isto é,

$$
\lim _{T \rightarrow \infty} E f_{x x}^{(T)}(\lambda)=f_{x x}(\lambda) .
$$

Assim, $f_{x x}^{(T)}(\lambda)$ é um estimador assintoticamente não viciado de $f_{x x}(\lambda)$.

Teorema 2.8: $\operatorname{Se} X(t), t=0, \pm 1, \cdots$, série real estacionária, $\lambda-\frac{2 \pi s(T)}{T}=$ $O\left(T^{-1}\right), \lambda \pm \mu \not \equiv 0(\bmod 2 \pi)$ e $m$ independe de $T$, então

(i) $\operatorname{Var} f_{x x}^{(T)}(\lambda)= \begin{cases}\frac{f_{x x}(\lambda)^{2}}{2 m+1}+O\left(T^{-1}\right) & \text { se } \lambda \not \equiv 0(\bmod \pi) \\ \frac{f_{x x}(\lambda)^{2}}{m}+O\left(T^{-1}\right) & \text { se } \lambda \equiv 0(\bmod \pi)\end{cases}$

(ii) $\operatorname{Cov}\left\{f_{x x}^{(T)}(\lambda), f_{x x}^{(T)}(\mu)\right\}=O\left(T^{-1}\right)$.

Teorema 2.9: $\quad$ Sob as condições do Teorema 2.3 e seja $s(T)$ um inteiro $\operatorname{com} \frac{2 \pi s(T)}{T} \rightarrow \lambda$ quando $T \rightarrow \infty, \lambda_{j} \pm \lambda_{k} \not \equiv 0(\bmod 2 \pi)$ para $1 \leq j<k \leq J$, então

(i) Para $j=1, \cdots, J, f_{x x}^{(T)}\left(\lambda_{j}\right) \stackrel{\mathcal{D}}{\rightarrow} f_{x x}\left(\lambda_{j}\right) \chi_{4 m+2}^{2} /(4 m+2)$ independentes se $\lambda_{j} \not \equiv 0(\bmod \pi) \mathrm{e}$ 
(ii) Para $j=1, \cdots, J, f_{x x}^{(T)}\left(\lambda_{j}\right) \stackrel{\mathcal{D}}{\rightarrow} f_{x x}\left(\lambda_{j}\right) \chi_{2 m}^{2} /(2 m)$ independentes se $\lambda_{j} \equiv$ $0(\bmod \pi)$.

Para mais detalhes, ver Brillinger(1981), Morettin(1979), Bloomfield(1976), Priestley(1981). 


\section{Capítulo 3}

\section{Transformada de Walsh e Periodograma de Walsh e Walsh-Fourier}

\subsection{Algumas Definições}

Definição 3.1: As funções de Rademacher são dadas por

$$
\begin{gathered}
r(0, \lambda)= \begin{cases}1 & \text { se } 0 \leq \lambda \leq 1 / 2 \\
-1 & \text { se } 1 / 2 \leq \lambda \leq 1\end{cases} \\
r(0, \lambda+k)=r(0, \lambda), \quad k=0,1,2,3, \cdots \\
r(n, \lambda)=r\left(0,2^{n} \lambda\right), \quad n=1,2,3, \cdots
\end{gathered}
$$

PROPRIEDADES:

(i) Essas funções formam um sistema ortonormal. isto é,

$$
\int_{0}^{1} r(n, \lambda) r(m, \lambda) d \lambda=\delta_{n m}
$$

onde $\delta_{n m}$ é uma função delta de Kronecher,

$$
\delta_{n m}= \begin{cases}1 & \text { se } n=m \\ 0 & \text { caso contrário. }\end{cases}
$$

(ii)

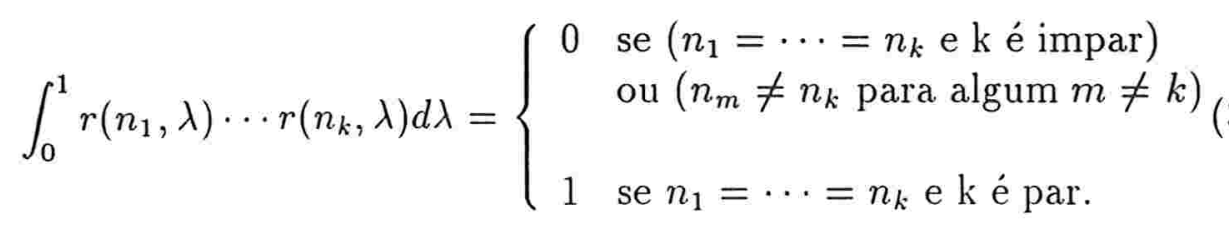


Agora, vamos denotar $\oplus$ como soma módulo 2 .

Definição 3.2: (a) Sejam $n, m$ inteiros não negativos. Consideremos suas expansões diádicas $n=\sum_{i=0}^{q} n_{i} 2^{i}, m=\sum_{i=0}^{q} m_{i} 2^{i}, n_{i}, m_{i} \in\{0,1\} \mathrm{e}$ $q \in \wp=\{0,1,2, \cdots\}$. A soma módulo 2 de $n$ e $m$ é dada por

$$
n \oplus m=\sum_{i=0}^{q}\left|n_{i}-m_{i}\right| 2^{i}
$$

(b) Sejam $x, y$ reais não negativos com expansões diádicas $x=\sum_{k=-\infty}^{\infty} x_{k} 2^{k}$, $y=\sum_{k=-\infty}^{\infty} y_{k} 2^{k}, x_{k}, y_{k} \in\{0,1\}$, então

$$
x \oplus y=\sum_{k=-\infty}^{\infty}\left(x_{k} \oplus y_{k}\right) 2^{k}
$$

onde

$$
\begin{aligned}
& 0 \oplus 0=1 \oplus 1=0, \\
& 1 \oplus 0=0 \oplus 1=1 .
\end{aligned}
$$

(c) Para reais negativos $-x,-y$, definimos:

$$
\begin{gathered}
(-x) \oplus y=x \oplus(-y)=-(x \oplus y), \\
(-x) \oplus(-y)=x \oplus y .
\end{gathered}
$$

Exemplo 3.1: Usando expansão diádica, $4=001,6=011$, então

$$
4 \oplus 6=001 \oplus 011=010=2 .
$$

Definição 3.3: $x$ é um racional diádico se sua expansão diádica é finita.

Definição 3.4: (a) As funções de Walsh $\{\Psi(n, \lambda), 0 \leq \lambda<1, n=$ $0,1,2, \cdots\}$ são dadas por

(i) $\Psi(0, \lambda)=1,0 \leq \lambda<1$. 
(ii) Se $n$ tem a expansão diádica $n=\sum_{i=0}^{\infty} \lambda_{i} 2^{i}$, e $\lambda_{i}=0$ para $i>m$, então

$$
\Psi(n, \lambda)=\prod_{i=1}^{r}\left\{r\left(m_{i}, \lambda\right)\right\}
$$

onde $m_{1}, \cdots, m_{r}$ correspondem aos coeficientes $\lambda_{m_{\mathbf{i}}}=1$ e $\{r(k, \lambda)\}$ são as funções de Rademacher.

\section{PROPRIEDADES:}

(i) O sistema $\{\Psi(n, \lambda)\}$ é ortonormal em $[0,1)$, isto é

$$
\int_{0}^{1} \Psi(n, \lambda) \Psi(m, \lambda) d \lambda=\delta_{n m}
$$

(ii) O sistema é completo, isto é, se $f(\lambda)$ é tal que

$$
\int_{0}^{1} f(\lambda) \Psi(n, \lambda) d \lambda=0, \quad n=0,1,2, \cdots,
$$

então $f(\lambda)=0$.

(iii) $\{\Psi(n, \lambda)\}$ é periódica, isto é,

$$
\Psi(n, \lambda+k)=\Psi(n, \lambda), \quad n, k=0,1,2, \cdots
$$

(iv) Para cada $y$ e quase todos $x$,

$$
\Psi(n, x \oplus y)=\Psi(n, x) \Psi(n, y),
$$

com exceção a $x$ tal que $(x \oplus y)$ é racional diádico.

(v) Para cada $y$ fixo e $f$ integrável,

$$
\int_{0}^{1} f(y \oplus x) d x=\int_{0}^{1} f(x) d x .
$$


(b) A função discreta de Walsh é dada por

$$
\Psi(n, m / T),
$$

onde $n, m=0,1,2, \cdots, T-1$ e $T=2^{p}$.

\section{PRopriedades:}

(i)

$$
\Psi(n, m / T)=\Psi(m, n / T) .
$$

(ii)

$$
\Psi\left(n_{1}, m / T\right) \Psi\left(n_{2}, m / T\right)=\Psi\left(n_{1} \oplus n_{2}, m / T\right) .
$$

(iii)

$$
\Psi\left(n, m_{1} / T\right) \Psi\left(n, m_{2} / T\right)=\Psi\left(n,\left(m_{1} \oplus m_{2}\right) / T\right) .
$$

(iv)

$$
T^{-1} \sum_{n=0}^{T-1} \Psi\left(n, \frac{m}{T}\right)=\delta_{0 m}, \quad m=0,1, \cdots, T-1 .
$$

(c) A função de Walsh generalizada é dada por

$$
\Psi(y, x)=\Psi([y], x) \Psi([x], y), x, y \in \Re_{+},
$$

onde $[x]$ e $[y]$ são os maiores inteiros em $x$ e $y$.

PROPRIEDADES:

(i) $\Psi(y, x)=\Psi(x, y)$.

(ii) $\Psi(y, x \oplus z)=\Psi(y, x) \Psi(y, z)$.

(iii) $\Psi\left(y_{1} \oplus y_{2}, x\right)=\Psi\left(y_{1}, x\right) \Psi\left(y_{2}, x\right)$.

As funções de $r(n, \lambda)$ e $\Psi(n, \lambda)$ estão apresentadas nas Figuras 3.1 e 3.2 . 

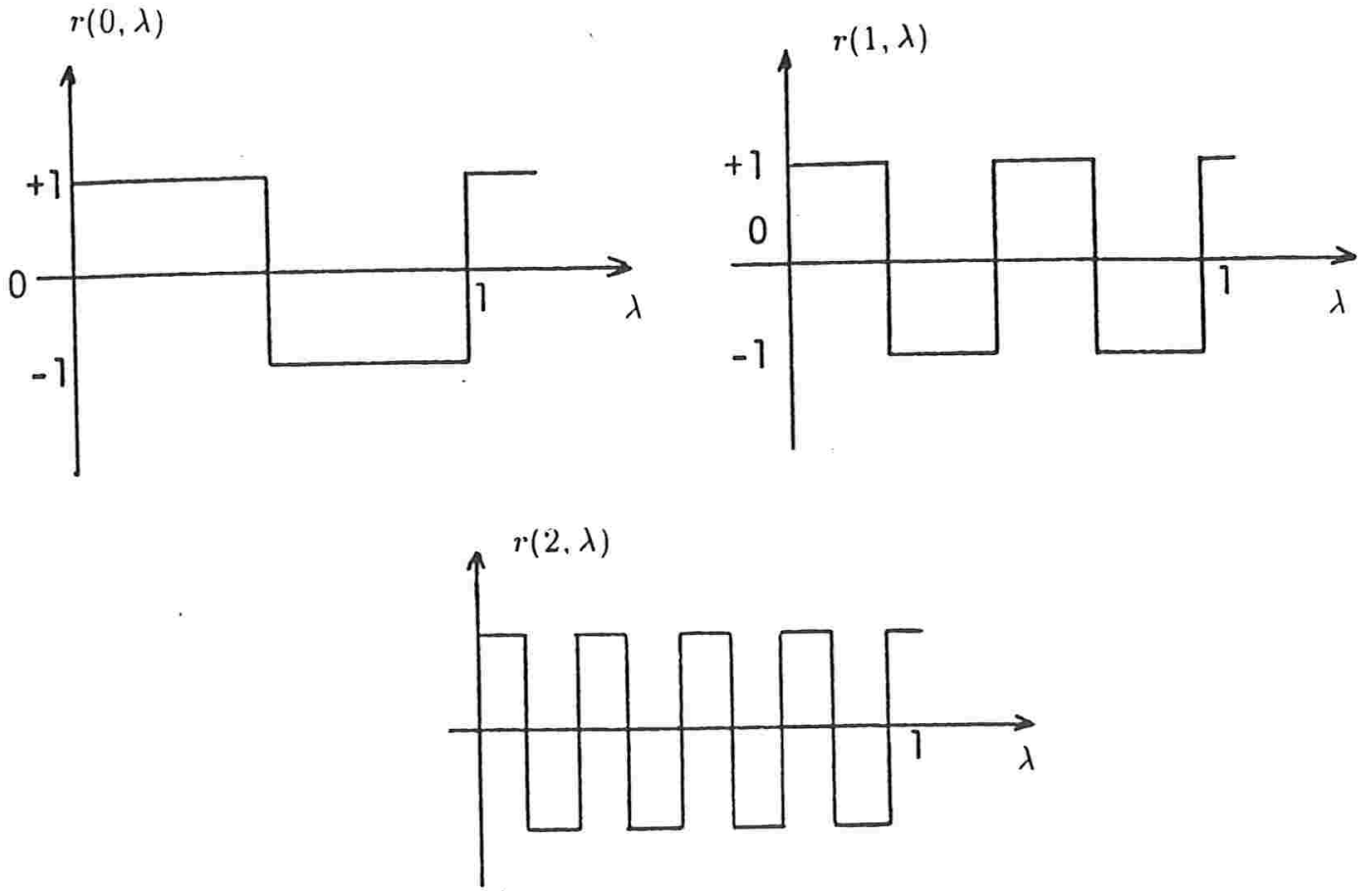

Figura 3.1: As funções de Rademacher
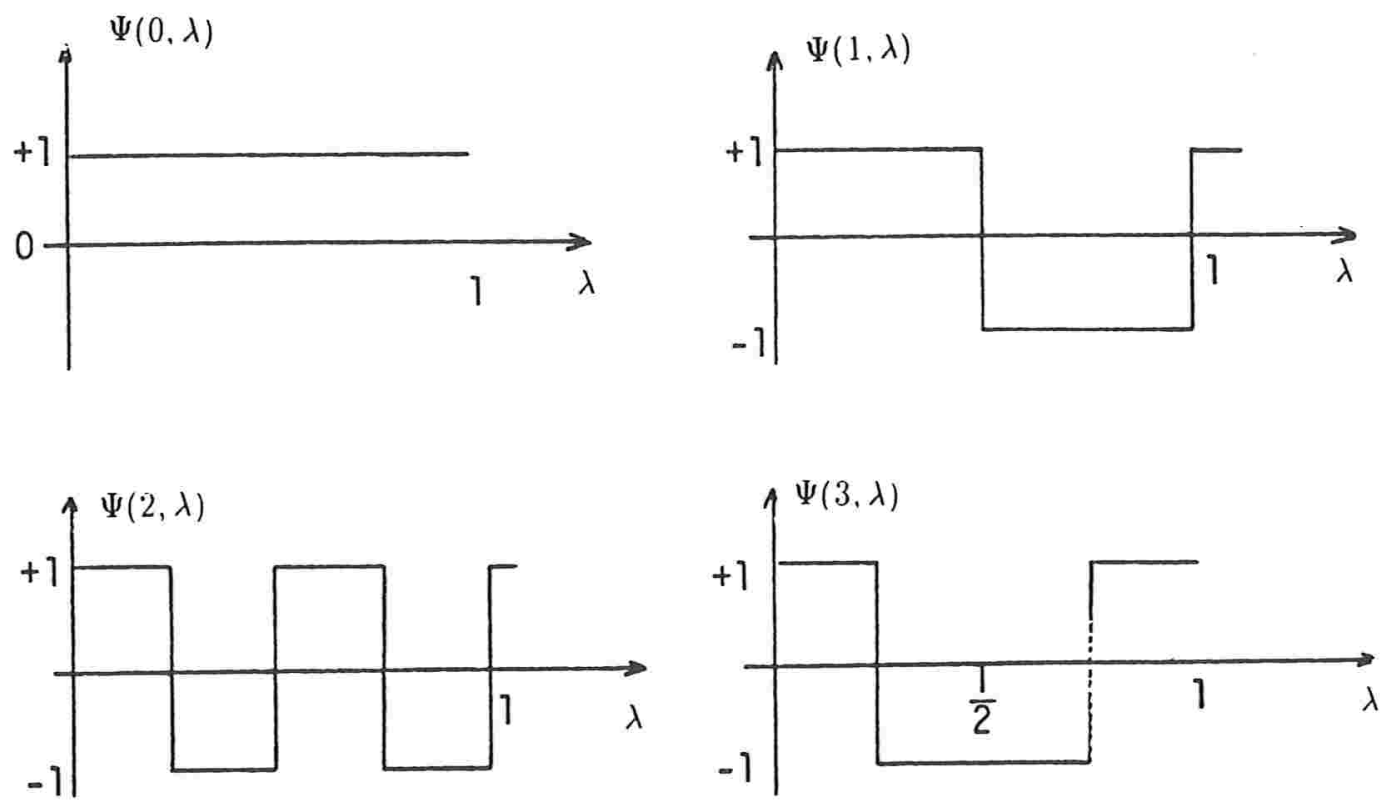

Figura 3.2: As funções de Walsh 
Definição 3.5: $\quad X=\{X(t), t \in \wp\}$ é um processo estacionário diádico se sua média é constante (sem perda de generalidade, igual a zero) e sua função de covariância

$$
B_{x x}(n, m)=\operatorname{Cov}(X(n), X(m))=E(X(n) X(m))
$$

depende somente de $(n \oplus m)$. Portanto, podemos escrever

$$
B_{x x}(\tau)=E(X(n) X(n \oplus \tau)), n, \tau \in \wp .
$$

ExEMPLo 3.2: $\{\varepsilon(t), t \in \wp\}$ sequência de variáveis independentes e identicamente distribuídas com $E(\varepsilon(t))=0$ e $\operatorname{Var}(\varepsilon(t))=\sigma^{2}$, para todos $t \in \wp$, então a função de covariância desse processo é dada por

$$
B(\tau)=E(\varepsilon(t) \varepsilon(t \oplus \tau))= \begin{cases}0 & \tau \neq 0 \\ \sigma^{2} & \tau=0\end{cases}
$$

EXEmplo 3.3: Suponha que $X(t)=\varepsilon \Psi(t, \lambda)$, com $\lambda$ constante e $\varepsilon$ variável aleatória $\operatorname{com} E(\varepsilon)=0$ e $\operatorname{Var}(\varepsilon)=\sigma^{2}$, então,

$$
E(X(t))=0
$$

$\mathrm{e}$

$$
\begin{aligned}
B(\tau) & =E(X(t \oplus \tau) X(t)) \\
& =E(\varepsilon \Psi(t \oplus \tau, \lambda) \varepsilon \Psi(t, \lambda)) \\
& =\Psi(t \oplus \tau, \lambda) \Psi(t, \lambda) E\left(\varepsilon^{2}\right) \\
& =\Psi(\tau, \lambda) \sigma^{2} .
\end{aligned}
$$

Definição 3.6: Seja $X=\{X(t), t \in \wp\}$ um processo estacionário diádico, com média zero e função de covariância $B_{x x}(\tau), \tau \in \wp$. O espectro de Walsh de $X$ é dado por

$$
f_{x x}^{(w)}(\lambda)=\sum_{\tau=0}^{\infty} B_{x x}(\tau) \Psi(\tau, \lambda), \quad 0 \leq \lambda<1
$$


se

$$
\sum_{\tau=0}^{\infty}\left|B_{x x}(\tau)\right|<\infty
$$

Exemplo 3.4: O espectro de Walsh de $\varepsilon=(\varepsilon(t), t \in \wp)$ é

$$
\begin{aligned}
f_{\varepsilon \varepsilon}^{(w)}(\lambda) & =\sum_{\tau=0}^{\infty} B_{\varepsilon \varepsilon}(\tau) \Psi(\tau, \lambda) \\
& =B_{\varepsilon \varepsilon}(0) \Psi(0, \lambda) \\
& =\sigma^{2} .
\end{aligned}
$$

Morettin(1972) demonstrou que $f_{x x}^{(w)}(\cdot)$ é periódico de período 1 , não negativo e uniformemente $W$-contínuo (Morgenthaler, 1957).

Definição 3.7: Suponha que $X=\{X(t), t=0, \pm 1, \cdots\}$ seja uma série temporal estacionária com média zero e funçio de covariância $\left\{R_{x x}(\tau)\right.$, $\tau=0, \pm 1, \cdots\}$ e, $T=2^{p}$, o tamanho da amostra. A j-ésima covariância lógica é dada por

$$
\Gamma_{x x}(j)=\frac{1}{T} \sum_{t=0}^{T-1} R_{x x}(j \oplus t-t)
$$

para $1 \leq j<T, \Gamma_{x x}(0)=R_{x x}(0)$.

Definição 3.8: Se $X(t)$ é uma série temporal estacionária tal que

$$
\sum_{j=0}^{\infty}\left|\Gamma_{x x}(j)\right|<\infty,
$$

então o espectro de Walsh-Fourier de $X(t)$ é dado por

$$
S_{x x}^{(w)}(\lambda)=\sum_{j=0}^{\infty} \Gamma_{x x}(j) \Psi(j, \lambda) .
$$

Usando alguns resultados de Fine(1957), Kohn(1980a) provou que $\Gamma_{x x}(j)$ tem uma representação do tipo

$$
\Gamma_{x x}(j)=\int_{0}^{1} \Psi(j, \lambda) d G(\lambda)
$$


onde $G(\cdot)$ é uma função não decrescente, contínua à direita com $G(0)=0$.

\subsection{Transformada Finita de Walsh-Fourier e Periodograma}

Seja $X=\{X(t), t \in T\}$ um processo estacionário se $T=\mathcal{Z}$ e um processo estacionário diádico se $T=\wp$. A transformada finita de Walsh-Fourier ou Walsh dos valores $X(0), X(1), \cdots, X(T-1)$ de $X(t)$ é

$$
d_{x}^{(T, w)}(\lambda)=\sum_{t=0}^{T-1} X(t) \Psi(t, \lambda), \quad 0 \leq \lambda<1,
$$

$\operatorname{com} T=2^{p}, p$ inteiro e não negativo.

Para as séries cujo tamanho não possa ser escrito na forma $2^{p}$, completaremos estas séries com sua média amostral até que seu tamanho atinja uma potência de 2 .

\section{PROPRIEDADES:}

(i) Para cada $t \leq T-1, \Psi(t, \lambda)$ é constante em cada subintervalo $(k / T,(k+$ $1) / T$ ) de $[0,1]$, assim a transformada finita de Walsh-Fourier $d^{(T, w)}(\cdot)$ assume somente $T$ valores diferentes, isto é,

$$
d^{(T, w)}(k)=d^{(T, w)}(\lambda)
$$

para $\frac{k}{T}<\lambda \leq \frac{k+1}{T}, k=0,1, \cdots, T-1$.

(ii) Nagai(1976) provou que $\left\{d^{(T, w)}(k), k=0,1, \cdots, T-1\right\}$ são ortogonais se e somente se $\{X(t), t \in T\}$ é estacionário diádico, com

$$
E\left[d^{(T, w)}(j) d^{(T, w)}(k)\right]= \begin{cases}\sigma^{2}(j) & \text { se } j=k \\ 0 & \text { se } j \neq k\end{cases}
$$

e

$$
\sigma^{2}(j)=T^{2} \int_{j / T}^{(j+1) / T} f_{x x}^{(w)}(\lambda) d \lambda, j=0,1, \cdots, T-1
$$


Teorema 3.1: Se $X(t)$ é estacionário diádico, (2.22) é válido e $\lambda_{j}+\lambda_{k} \notin$ $V(t)(\bmod 1), V(t)=\left[0, T^{-1}\right]=\left[0,2^{-t}\right]$ e $1 \leq j<k \leq M$, então as variáveis aleatórias $d_{x}^{(T, w)}\left(\lambda_{1}\right), \cdots, d_{x}^{(T, w)}\left(\lambda_{M}\right)$ são assintoticamente independentes com distribuições assintóticas $N\left(0, T f_{x x}^{(w)}\left(\lambda_{1}\right)\right), \cdots, T\left(0, T f_{x x}^{(w)}\left(\lambda_{M}\right)\right)$, onde $f_{x x}^{(w)}(\cdot)$ é o espectro de Walsh de $X(t)$ definido por (3.21).

Demonstração, ver Morettin(1972).

Resultado (Kohn, 1980a): Se $X(t)$ é estacionário e

$$
\lim _{n \rightarrow \infty} \sum_{|j|<2^{n}}\left(1-|j| / 2^{n}\right)\left|R_{x x}(j)\right|<\infty
$$

então $\sum_{j=0}^{\infty}\left|\Gamma_{x x}(j)\right|<\infty$ e portanto existe o espectro de Walsh-Fourier $S_{x x}^{(w)}(\lambda)$. Além disso,

$$
\operatorname{Var}\left[T^{-1 / 2} d_{x}^{(T, w)}(\lambda)\right] \rightarrow S_{x x}^{(w)}(\lambda), \quad T \rightarrow \infty .
$$

Teorema 3.2: Suponha que $X(t)$ um processo estritamente estacionário, $\Im_{t}$ a $\sigma$-álgebra gerada por $\{X(j), j \leq t\}$ e

$$
\alpha_{j}=\left[E\left\{E\left(X(t) \mid \Im_{t-j}\right)-E\left(X(t) \mid \Im_{t-j-1}\right)\right\}^{2}\right]^{1 / 2}, j \geq 0 .
$$

$\mathrm{Se}$

$$
\sum_{j=0}^{\infty} \alpha_{j}<\infty,
$$

então $d_{x}^{(T, w)}(\lambda)$ é assintoticamente $N\left(0, T S_{x x}^{(w)}(\lambda)\right)$.

Observação: Assume-se que $\Im_{-\infty}$ é trivial e o Teorema 3.2 vale para $X(T)$ racional diádico com $X(T) \oplus X(t) \rightarrow 0$ quando $T \rightarrow \infty$.

Ver Kohn(1980a) e Morettin(1981) para mais detalhes.

Seja $\{X(t), t=0,1, \cdots\}$ um processo estacionário diádico e $d_{x}^{(T, w)}(\cdot)$ é a transformada finita de Walsh dos valores $X(0), X(1), \cdots, X(T-1)$. $\mathrm{O}$ Teorema 3.1 sugere que $f_{x x}^{(w)}(\lambda)$ definido por (3.21) pode ser estimado por

$$
I_{x x}^{(T, w)}(\lambda)=T^{-1}\left[d_{x}^{(T, w)}(\lambda)\right]^{2},
$$


que é chamado periodograma de Walsh.

Teorema 3.3: Segundo (3.28) temos que

$$
I_{x x}^{(T, w)}(\lambda)=I_{x x}^{(T, w)}(k)
$$

para $k / T \leq \lambda \leq(k+1) / T, k=0,1, \cdots, T-1$. Deste resultado temos que

$$
E\left\{I_{x x}^{(T, w)}(\lambda)\right\}=T^{-1} E\left\{d_{x}^{(T, w)}(\lambda)\right\}^{2} \rightarrow f_{x x}^{(w)}(\lambda),
$$

quando $T \rightarrow \infty$. Ou seja, o estimador $I_{x x}^{(T, w)}(\lambda)$ é assintoticamente nãoviciado.

Teorema 3.4: (a) Se $\sum_{\tau} \tau\left|B_{x x}(\tau)\right|<\infty$, então

$$
E\left\{I_{x x}^{(T, w)}(\lambda)\right\}=f_{x x}^{(w)}(\lambda)+O\left(T^{-1}\right)
$$

(b)

$$
\operatorname{Cov}\left\{I_{x x}^{(T, w)}(\lambda), I_{x x}^{(T, w)}(\mu)\right\}=2 T^{-2}\left[D_{T}(\lambda \oplus \mu)\right]^{2}\left[f_{x x}^{(w)}\right]^{2}+O\left(T^{-1}\right),
$$

onde $D_{T}(\lambda \oplus \mu)$ é núcleo de Dirichlet,

$$
D_{T}(\lambda \oplus \mu)= \begin{cases}T & \text { se } 0 \leq \lambda \oplus \mu \leq T^{-1} \\ 0 & \text { caso contrário. }\end{cases}
$$

c)

$$
\operatorname{Var}\left\{I_{x x}^{(T, w)}(\lambda)\right\}=2\left[f_{x x}^{(w)}(\lambda)\right]^{2}+O\left(T^{-1}\right)
$$

Assim, de acordo com o Teorema 3.4, verifica-se que o periodograma de Walsh não é um estimador consistente de $f_{x x}^{(w)}(\lambda)$.

Teorema 3.5: Se $X(t)$ é um processo estacionário diádico, (2.22) é válido, $\lambda_{j}+\lambda_{k} \notin V(t)(\bmod 1), V(t)=\left[0, T^{-1}\right]=\left[0,2^{-t}\right]$ e $1 \leq j<k \leq M$, então $I_{x x}^{(T, w)}\left(\lambda_{j}\right) \stackrel{\mathcal{D}}{\rightarrow} f_{x x}^{(w)}\left(\lambda_{j}\right) \chi^{2}(1)$ independentes.

Detalhes, ver Morettin(1974a, 1974b). 
Agora, consideremos $X(t)$ uma série estacionária e vamos definir, como um estimador de $S_{x x}^{(w)}(\lambda)$, o periodograma de Walsh-Fourier

$$
I_{x x}^{(T, s)}(\lambda)=T^{-1}\left[d_{x}^{(T, w)}(\lambda)\right]^{2} .
$$

Teorema 3.6: Se $X(t)$ é um processo estritamente estacionário e (3.32) é válido, então

$$
\operatorname{Var}\left\{I_{x x}^{(T, s)}(\lambda)\right\} \rightarrow 2\left[S_{x x}^{(w)}(\lambda)\right]^{2},
$$

quando $T \rightarrow \infty$, e $I_{x x}^{(T, s)}(\lambda)$ é assintoticamente $S_{x x}^{(w)}(\lambda) \chi^{2}(1)$.

O Teorema 3.6 também mostra que o periodograma de Walsh-Fourier não é um estimador consistente.

Devido a grande instabilidade dos periodogramas de Walsh e WalshFourier (estimadores não consistente do espectro), os Teoremas 3.5 e 3.6 sugerem construir estimadores espectrais com propriedades melhores.

De acordo com o Teorema 3.5, se $X(t)$ é estacionário diádico e $T=L K$, onde $K=2^{p}$, então o periodograma suavizado de Walsh é dado por

$$
I_{x x}^{(K, w)}(\lambda, l)=K^{-1}\left[\sum_{t=0}^{K-1} X(t+l K) \Psi(t+l K, \lambda)\right]^{2},
$$

para $l=0,1, \cdots, L-1$, e um novo estimador para $f_{x x}^{(w)}(\lambda)$ é obtido por

$$
f_{x x}^{(T, w)}(\lambda)=L^{-1} \sum_{l=0}^{L-1} I_{x x}^{(K, w)}(\lambda, l)
$$

Teorema 3.7: Se $X(t)$ é estacionário diádico e $T=L K$, onde $K=2^{p}$, então

(i) para $l=0,1, \cdots, L-1$ e $T \rightarrow \infty, I_{x x}^{(K, w)}(\lambda, l) \stackrel{\mathcal{D}}{\rightarrow} f_{x x}^{(w)}(\lambda) \chi^{2}(1)$ independentes e

(ii) para L fixo e $T \rightarrow \infty, f_{x x}^{(T, w)}(\lambda) \stackrel{\mathcal{D}}{\rightarrow} f_{x x}^{(w)}(\lambda) \chi^{2}(L) / L$. 
Para demonstração, ver Morettin(1976).

De acordo com o Teorema 3.6, se $X(t)$ é uma série estacionária e $T=L K$, onde $K=2^{p}$, então o periodograma suavizado de Walsh-Fourier é dado por

$$
I_{x x}^{(K, s)}(\lambda, l)=K^{-1}\left[\sum_{t=0}^{K-1} X(t+l K) \Psi(t+l K, \lambda)\right]^{2},
$$

para $l=0,1, \cdots, L-1$, e um novo estimador para $S_{x x}^{(w)}(\lambda)$ é obtido por

$$
S_{x x}^{(T, s)}(\lambda)=L^{-1} \sum_{l=0}^{L-1} I_{x x}^{(K, s)}(\lambda, l),
$$

onde $I_{x x}^{(K, s)}(\lambda, l)$ é definido por (3.42).

Teorema 3.8: Se $X(t)$ é uma série estacionária e $T=L K$, onde $K=2^{p}$, então

(i) para $l=0,1, \cdots, L-1$ e $T \rightarrow \infty, I_{x x}^{(K, s)}(\lambda, l) \stackrel{\mathcal{D}}{\rightarrow} S_{x x}^{(w)}(\lambda) \chi^{2}(1)$ independentes e

(ii) para L fixo e $T \rightarrow \infty, S_{x x}^{(T, s)}(\lambda) \stackrel{\mathcal{D}}{\rightarrow} S_{x x}^{(w)}(\lambda) \chi^{2}(L) / L$.

Detalhes, ver Stoffer(1985), Morettin(1972, 1983). 


\section{Capítulo 4}

\section{Análise de Variância Usando a Transformada de Fourier}

\subsection{Introdução}

Neste capítulo, analisaremos as variações apresentadas nos dados cujas medidas respostas são séries temporais. As análises propostas podem ser vistas como extensões de procedimentos usados na ANOVA (Análise de Variância), quando cada ocorrência (ou série) de um planejamento conduz a uma variável real. Nosso objetivo consiste na aplicação de uma versão complexa de ANOVA aos componentes das transformadas discretas de Fourier das séries observadas. Serão considerados efeitos fixo e aleatório.

Uma abordagem comum da análise de variância é feita através de um modelo de regressão linear com a seguinte forma (ver Roy et al, 1971)

$$
Y=Z \Theta X+\epsilon
$$

onde

Y é uma matriz de dimensão $N$ x $T$ das respostas observadas, onde $\mathrm{N}$ linhas correspondem aos indivíduos do experimento e $\mathrm{T}$ ao número de respostas de cada indivíduo;

$Z$ é uma matriz de planejamento entre os indivíduos, de dimensão $N \times M$;

$X$ é uma matriz de planejamento dentro dos indivíduos, de dimensão $S \times T$;

$\Theta$ é uma matriz de coeficientes não observados (parâmetros), de dimensão $M \times S$;

$\epsilon$ é uma matriz de erros estocásticos, de dimensão $N \times T$. 
Geralmente, a matriz de planejamento $Z$ é construída com $0^{\prime}$ s e 1 's e $Z$ e $X$ são consideradas na maioria das vezes como fixas. A matriz $\Theta$ é considerada fixa no modelo de efeitos fixos e estocástica no modelo de efeitos aleatórios. Nosso objetivo é verificar se algum elemento de $\Theta$ é zero, estimar os parâmetros de interesse e calcular os erros das estimativas.

O modelo (4.1) pode ser reescrito como

$$
Y_{n}(t)=\sum_{j=1}^{S} \sum_{i=1}^{M} Z_{n i} \Theta_{i j} X_{j}(t)+\epsilon_{n}(t),
$$

onde $Y_{n}(t)$ representa o elemento na n-ésima linha e t-ésima coluna da matriz $Y$.

Neste capítulo, estudaremos o caso em que $Y_{n}(t), t=0, \cdots, T-1$, são $N$ séries temporais.

Para fazermos uma análise de variância, quando temos como medidas respostas um conjunto de $N$ séries temporais estacionárias, $\left\{X_{n}(t), t=\right.$ $0, \cdots, T-1$ e $n=1, \cdots, N\}$, utilizaremos como ferramenta básica a transformada discreta de Fourier de cada uma delas,

$$
d_{x_{n}}^{(T)}(k)=\sum_{t=0}^{T-1} X_{n}(t) \exp \{-i 2 \pi k t / T\}
$$

para $k=0,1, \cdots, \frac{[T-1]}{2}$.

Segundo o Teorema 2.1 , para $k=1, \cdots, K, K \ll T, \frac{2 \pi k}{T} \simeq \lambda$ e $\frac{2 \pi k}{T} \neq$ $0, \pm \pi, \cdots,(4.3)$ convergem em distribuição para

$$
N^{c}\left(0,2 \pi T f_{x_{n} x_{n}}(\lambda)\right)
$$

independentes, onde $f_{x_{n} x_{n}}(\lambda)$ é o espectro da série $X_{n}(t)$. Portanto,

$$
X_{n}^{\wedge}(k)=(2 \pi T)^{-1 / 2} d_{x_{n}}^{(T)}(k)
$$

têm as seguintes representações

$$
X_{n}^{\wedge}(k)=\xi_{n k}+O_{a . s .}(1),
$$


para todo $\mathrm{n}$, onde $\xi_{n 1}, \cdots, \xi_{n K}$ são variáveis $N^{c}\left(0, f_{x_{n} x_{n}}(\lambda)\right)$ independentes e $O_{\text {a.s. }}(1) \rightarrow 0$ quase certamente quando $T \rightarrow \infty$.

Vimos no Capítulo 2, que um estimador padronizado de $f_{x_{n} x_{n}}(\lambda)$, denominado periodograma suavizado, é dado por

$$
f_{x_{n} x_{n}}^{(T)}(\lambda)=K^{-1} \sum_{k=1}^{K}\left|X_{n}^{\wedge}(k)\right|^{2} .
$$

Segundo o Teorema 2.9, (4.7) tem a seguinte representação

$$
f_{x_{n} x_{n}}^{(T)}(\lambda)=f_{x_{n} x_{n}}(\lambda) \chi_{2 K}^{2} / 2 K+O_{\text {a.s. }}(1)
$$

para todo $\mathrm{n}$.

Na prática, o problema principal é a escolha do valor $K$. Queremos que $K$ seja grande de forma a estabilizar o estimador indicado por (4.8), mas, por outro lado, um valor muito grande de $K$, aumentará muito o viés do estimador. Ver Skorokhod(1956), Hannan(1970) e Brillinger(1981).

Alguns casos particulares do modelo (4.2) serão tratados nas seções seguintes.

\subsection{Modelo com um sinal comum}

Um importante caso especial da família ANOVA é detectar um sinal comum $\Omega(t)$, possivelmente obscurecido pelo erro, em uma coleção de $\mathrm{N}$ séries temporais. Neste caso, podemos usar o modelo

$$
Y_{n}(t)=\dot{\mu}_{n}+\Omega(t)+\epsilon_{n}(t)
$$

para $n=1, \cdots, N, t=0,1, \cdots, T-1$.

\subsubsection{O modelo com sinal determinístico}

Suponha que queremos detectar um comportamento padrão para o eletrocorticograma medido no córtex frontal de ratos normais durante o estado 
do sono paradoxal. Seja $Y_{n}(t), n=1, \cdots, N, t=0, \cdots, T-1$, o eletrocorticograma do n-ésimo rato no instante t. Assim podemos modelar os dados pelo modelo (4.9), onde

$\mu_{n}$ é a média do eletrocorticograma do n-ésimo rato e é constante;

$\Omega(t)$ representa o sinal comum a todos os ratos e é determinístico;

$\epsilon_{n}(\cdot)$ são séries estacionárias, independentes com média zero e função de covariância $c_{\epsilon \epsilon}(u)$ e espectro $f_{\epsilon \epsilon}(\lambda)$.

Segundo as suposições do modelo, temos

$$
E Y_{n}(t)=\mu_{n}+\Omega(t)
$$

e

$$
\operatorname{Cov}\left\{Y_{n}(t+u), Y_{n}(t)\right\}=c_{\epsilon \epsilon}(u)
$$

Testar a existência de um sinal comum é equivalente a testar a hipótese

$$
H_{01}: \Omega(t)=0,
$$

que no nosso exemplo significa verificar se existe um sinal comum a todos os ratos.

Pela definição (4.5) e a representação (4.6), o modelo (4.9) pode ser reescrito como

$$
Y_{n}^{\wedge}(k)=\Omega^{\wedge}(k)+\xi_{n k}+O_{a . s .}(1),
$$

para $k=0, \cdots, \frac{[T-1]}{2}$, com $\xi_{n k}$ variáveis $N^{c}\left(0, f_{\epsilon \epsilon}(\lambda)\right)$ independentes.

Para fazer o teste (4.10) consideraremos as seguintes somas de quadrados:

$$
S Q H_{01}(\lambda)=N \sum_{k=1}^{K}\left|Y_{.}^{\wedge}(k)\right|^{2}
$$

e

$$
S Q R_{01}(\lambda)=\sum_{n=1}^{N} \sum_{k=1}^{K}\left|Y_{n}^{\wedge}(k)-Y_{.}^{\wedge}(k)\right|^{2}
$$


onde

$$
Y^{\wedge}(k)=N^{-1} \sum_{n=1}^{N} Y_{n}^{\wedge}(k)
$$

Utilizando a expressão (4.11) e a extensão complexa do teorema de FisherCochran dado no Capítulo 2 (ver a propriedade V da definição 2.10(b)), (4.12) e (4.13) convergem quase certamente para

$$
f_{\epsilon \epsilon}(\lambda) \chi_{2 K}^{2}\left(\frac{N \sum_{k}\left|\Omega^{\wedge}(k)\right|^{2}}{f_{\epsilon \epsilon}(\lambda)}\right) / 2
$$

e

$$
f_{\epsilon \epsilon}(\lambda) \chi_{2 K(N-1)}^{2} / 2
$$

respectivamente, $\operatorname{com} \chi_{\nu}^{2}$ independentes.

Utilizando (4.7), podemos calcular os valores esperados de (4.12) e (4.13)

$$
\begin{aligned}
E\left(S Q H_{01}(\lambda)\right) & =E\left\{N \sum_{k=1}^{K}\left|\Omega^{\wedge}(k)+\xi \cdot k\right|^{2}\right\} \\
& =E\left\{N \sum_{k=1}^{K}\left|\Omega^{\wedge}(k)\right|^{2}+N K \frac{f_{\epsilon \epsilon}^{(T)}(\lambda)}{N}\right\} \\
& =N \sum_{k=1}^{K}\left|\Omega^{\wedge}(k)\right|^{2}+K f_{\epsilon \epsilon}(\lambda) \\
& =N K f_{\Omega \Omega}^{(T)}(\lambda)+K f_{\epsilon \epsilon}(\lambda),
\end{aligned}
$$

onde $\xi_{\cdot k}=\frac{\sum_{n=1}^{N} \xi_{n k}}{N} \mathrm{e}$

$$
\begin{aligned}
E\left(S Q R_{01}(\lambda)\right) & =E\left\{\sum_{n=1}^{N} \sum_{k=1}^{K}\left|\xi_{n k}-\xi_{\cdot k}\right|^{2}\right\} \\
& =E\left\{\sum_{n=1}^{N} K\left(f_{\epsilon \epsilon}^{(T)}(\lambda)-\frac{f_{\epsilon \epsilon}^{(T)}(\lambda)}{N}\right)\right\} \\
& =\sum_{n=1}^{N} K E\left\{\frac{N-1}{N} f_{\epsilon \epsilon}^{(T)}(\lambda)\right\} \\
& =K(N-1) f_{\epsilon \epsilon}(\lambda)
\end{aligned}
$$


O teste para (4.10) é realizado utilizando a estatística

$$
F_{01}(\lambda)=\frac{2 S Q H_{01}(\lambda) / 2 K}{2 S Q R_{01}(\lambda) / 2 K(N-1)}
$$

que tem uma distribuição F com parâmetro de não centralidade $\frac{N \sum_{k}\left|\Omega^{\wedge}(k)\right|^{2}}{f_{\epsilon e}(\lambda)}$. Sob $H_{01},(4.17)$ tem uma distribuição $\mathrm{F}(2 K, 2 K(N-1))$ para $0 \leq \lambda<\pi$. Ver Brillinger(1980).

Os resultados obtidos estão resumidos na Tabela 4.1: ANOVAF (Análise de Variância usando Fourier).

Tabela 4.1: ANOVAF para o teste (4.10) na frequência $\lambda$

\begin{tabular}{lcc}
\hline \hline fonte & soma de quadrados & grau de liberdade \\
& $(S Q)$ & $(g l)$ \\
\hline$\Omega(t)$ & $N \sum_{k}\left|Y_{.}^{\wedge}(k)\right|^{2}$ & $2 K$ \\
resíduo & $\sum_{k} \sum_{1}^{N}\left|Y_{n}^{\wedge}(k)-Y_{\cdot}^{\wedge}(k)\right|^{2}$ & $2 K(N-1)$ \\
\hline Total & $\sum_{k} \sum_{1}^{N}\left|Y_{n}^{\wedge}(k)\right|^{2}$ & $2 K N$ \\
\hline \hline
\end{tabular}

\begin{tabular}{lccl}
\hline \hline fonte & quadrado médio $=2 S Q / g l$ & estatística & quadrado médio esperado \\
& $(Q M)$ & $F$ & $(E Q M)$ \\
\hline$\Omega(t)$ & $\frac{N \sum_{k}\left|Y^{\wedge}(k)\right|^{2}}{K}(\star)$ & $(\star) /(\star \star)$ & $f_{\epsilon \epsilon}(\lambda)+N f_{\Omega \Omega}^{(T)}(\lambda)$ \\
resíduo & $\frac{\sum_{k} \sum_{l_{1}^{N}\left|Y_{n}^{\wedge}(k)-Y^{\wedge}(k)\right|^{2}}^{K(N-1)}(\star \star)}{}$ & $f_{\epsilon \epsilon}(\lambda)$ \\
\hline Total & & \\
\hline \hline
\end{tabular}

Se quisermos usar os componentes espectrais não suavizados para testar (4.10) na frequência $\lambda_{k}=\frac{2 \pi k}{T}, k=0,1, \cdots, \frac{[T-1]}{2}$, utilizaremos a estatística

$$
F_{01}\left(\lambda_{k}\right)=\frac{N\left|Y_{.}^{\wedge}(k)\right|^{2}}{\sum_{n=1}^{N}\left|Y_{n}^{\wedge}(k)-Y^{\wedge}(k)\right|^{2} /(N-1)}
$$


que tem uma distribuição F com parâmetro de nüo centralidade $\frac{N\left|\Omega^{\wedge}(k)\right|^{2}}{f_{\epsilon \epsilon}\left(\lambda_{k}\right)}$ e que sob $H_{01}$, tem uma distribuição $\mathrm{F}(2,2(N-1))$. Ver Shumway(1988).

Se desejarmos testar várias frequências conjuntamente, podemos aplicar a analogia feita em análise multivariada utilizando uma das seguintes estatísticas globais

$$
\begin{aligned}
& \sup _{q=1}^{Q / 2} F(2 \pi q / Q), \\
& \sum_{q=1}^{Q / 2} F(2 \pi q / Q)
\end{aligned}
$$

$\mathrm{e}$

$$
\prod_{q=1}^{Q / 2}[1+(N-1) F(2 \pi q / Q)],
$$

para algum Q (número de frequências testadas), onde $F(\lambda)$ denota a estatística F da Tabela 4.1. Ver Roy et al(1971).

A estatística $Y^{\wedge}(k)$ é um estimador de $\Omega^{\wedge}(k)$ com média e variância dadas por

$$
E\left(Y^{\wedge}(k)\right)=\Omega^{\wedge}(k)
$$

$\mathrm{e}$

$$
\operatorname{Var}\left(Y_{.}^{\wedge}(k)\right)=f_{\epsilon \epsilon}(2 \pi k / T) .
$$

Dessa forma, o sinal $\Omega(t)$ pode ser estimado por $Y .(t)=\frac{\sum_{N} Y_{n}(t)}{N}$ com as seguintes propriedades

$$
E(Y .(t))=\mu .+\Omega(t)
$$

e

$$
\operatorname{Var}(Y .(t))=c_{\epsilon \epsilon}(0) / N
$$

\subsubsection{O modelo com sinal estocástico}

Brillinger(1973), apresenta um exemplo onde o interesse é analisar a similaridade climática das estações americanas. Foram selecionadas aleatoriamente $N=5$ estações e utilizado o modelo (4.9),

$$
Y_{n}(t)=\mu_{n}+\Omega(t)+\epsilon_{n}(t),
$$

onde 
$Y_{n}(t)$ é a temperatura mensal da n-ésima estação, $n=1, \cdots, N$;

$\mu_{n}$ é a média da temperatura mensal da n-ésima estação e é constante;

$\Omega(\cdot)$ representa o componente climático comum a todas as estações americanas e é uma série temporal estacionária com média zero, $\operatorname{Cov}\{\Omega(t+$ $u), \Omega(t)\}=c_{\Omega \Omega}(u)$ e espectro $f_{\Omega \Omega}(\lambda), u=0, \pm 1, \cdots ;$

$\epsilon_{n}(\cdot)$, são séries temporais estacionárias, independentes com média zero, $\operatorname{Cov}\left\{\epsilon_{n}(t+u), \epsilon_{n}(t)\right\}=c_{\epsilon \epsilon}(u)$ e espectro $f_{\epsilon \epsilon}(\lambda) ;$

$\Omega(\cdot)$ e $\epsilon_{n}(\cdot)$ são independentes.

Segundo as suposições do modelo, temos

$$
\begin{gathered}
E Y_{n}(t)=\mu_{n}, \\
\operatorname{Cov}\left\{Y_{n}(t+u), Y_{n}(t)\right\}=c_{\Omega \Omega}+c_{\epsilon \epsilon}(u), \\
f_{Y_{n} Y_{n}}(\lambda)=f_{\Omega \Omega}(\lambda)+f_{\epsilon \epsilon}(\lambda), \\
\operatorname{Cov}\left\{Y_{n}(t+u), Y_{n^{\prime}}(t)\right\}=c_{\Omega \Omega}(u), \quad n \neq n^{\prime}
\end{gathered}
$$

e

$$
f_{Y_{n} Y_{n^{\prime}}}=f_{\Omega \Omega}(\lambda)
$$

Sob estas condições, a expressão (4.11) continua valendo, mas pode ser melhor representada por

$$
Y_{n}^{\wedge}(k)=\gamma_{k}+\xi_{n k}+O_{a . s .}(1)
$$

onde

(i) $\gamma_{k}$ é uma variável $N^{c}\left(0, f_{\Omega \Omega}(\lambda)\right)$;

(ii) $\xi_{n k}$ são variáveis $N^{c}\left(0, f_{\epsilon \epsilon}(\lambda)\right)$ independentes;

(iii) $\gamma_{k}$ e $\xi_{n k}$ são independentes.

Neste caso, utilizando a expressão (4.22) e a extensão complexa do teorema de Fisher-Cochran (ver Capítulo 2), as somas de quadrados (4.12) e (4.13) convergem quase certamente para

$$
\left(N f_{\Omega \Omega}(\lambda)+f_{\epsilon \epsilon}(\lambda)\right) \chi_{2 K}^{2} / 2
$$




$$
f_{\epsilon \epsilon}(\lambda) \chi_{2 K(N-1)}^{2} / 2,
$$

respectivamente, com $\chi_{\nu}^{2}$ independentes. Testar (4.10) é equivalente a testar a hipótese

$$
H_{02}: f_{\Omega \Omega}(\cdot)=0,
$$

que no exemplo significa verificar a existência de uma similaridade climática a todas as estações.

De acordo com (4.23) e (4.24), o teste para (4.25) pode ser realizado utilizando a estatística (4.17) ou (4.18).

Os resultados obtidos estão resumidos na Tabela 4.2.

Tabela 4.2: ANOVAF para o teste (4.25) na frequência $\lambda$

\begin{tabular}{lcc}
\hline \hline fonte & soma de quadrados & grau de liberdade \\
& $(S Q)$ & $(g l)$ \\
\hline$\Omega(t)$ & $N \sum_{k}\left|Y^{\wedge}(k)\right|^{2}$ & $2 K$ \\
resíduo & $\sum_{k} \sum_{1}^{N}\left|Y_{n}^{\wedge}(k)-Y^{\wedge}(k)\right|^{2}$ & $2 K(N-1)$ \\
\hline Total & $\sum_{k} \sum_{1}^{N}\left|Y_{n}^{\wedge}(k)\right|^{2}$ & $2 K N$ \\
\hline \hline
\end{tabular}

\begin{tabular}{lccl}
\hline \hline fonte & quadrado médio=2SQ/gl & estatística & quadrado médio esperado \\
& $(Q M)$ & $F$ & $(E Q M)$ \\
\hline$\Omega(t)$ & $\frac{N \sum_{k}\left|Y^{\wedge}(k)\right|^{2}}{K}(\star)$ & $(\star) /(\star \star)$ & $f_{\epsilon \epsilon}(\lambda)+N f_{\Omega \Omega}(\lambda)$ \\
resíduo & $\frac{\sum_{k} \frac{\sum_{l}^{N}\left|Y_{n}^{\wedge}(k)-Y^{\wedge}(k)\right|^{2}}{K(N-1)}(\star \star)}{}$ & $f_{\epsilon \epsilon}(\lambda)$ \\
\hline Total & & & \\
\hline \hline
\end{tabular}


Pela Tabela 4.2, um estimador de $f_{\Omega \Omega}(\lambda)$, na frequência $\lambda$, pode ser obtido por

$$
f_{\Omega \Omega}^{(T)}(\lambda)=\frac{\sum_{k}\left|Y_{.^{\wedge}}(k)\right|^{2}}{K}-\frac{\sum_{k} \sum_{n}\left|Y_{n}^{\wedge}(k)-Y_{.}^{\wedge}(k)\right|^{2}}{N(N-1) K}
$$

com

$$
E\left\{f_{\Omega \Omega}^{(T)}(\lambda)\right\}=f_{\Omega \Omega}(\lambda)
$$

e

$$
\operatorname{Var}\left\{f_{\Omega \Omega}^{(T)}(\lambda)\right\}=\frac{\left(f_{\Omega \Omega}(\lambda)+f_{\epsilon \epsilon}(\lambda) / N\right)^{2}}{K}+\frac{f_{\epsilon \epsilon}^{2}(\lambda)}{N^{3} K} .
$$

Neste caso, as estatísticas (4.19), (4.20) e (4.21) também podem ser utilizadas para testar várias frequências conjuntamente.

\subsection{Modelo com um fator}

Suponhamos que um experimento seja realizado de tal forma que $\mathrm{M}$ tratamentos diferentes são aplicados e cujos resultados são séries temporais estacionárias. Um modelo apropriado para esta situação é

$$
Y_{l m}(t)=\mu_{l m}+\Omega(t)+\Gamma_{m}(t)+\epsilon_{l m}(t)
$$

para $l=1, \cdots, L_{m}, m=1, \cdots, M, N=L_{1}+\cdots+L_{M}, t=0,1, \cdots, T-1$, onde

$L_{m}$ : número de séries submetidas ao m-ésimo tratamento;

$\Gamma_{m}(t)$ : efeito do m-ésimo tratamento;

$\Omega(t)$ : sinal comum entre todas as séries observadas.

\subsubsection{O modelo com fator fixo}

Para exemplificar esse tipo de modelo, suponha que estamos interessados em verificar se existe diferença de comportamento entre os eletrocorticogramas medido no córtex frontal de ratos normais durante o estado de sono paradoxal e de alerta; também é de interesse verificar a existência de um 
comportamento comum para os dois estados. Consideremos $Y_{l m}(t)$, o eletrocorticograma do l-ésimo rato no m-ésimo estado, e podemos modelar os dados pelo modelo (4.27), onde

$\mu_{l m}$ é a média do eletrocorticograma do l-ésimo rato no m-ésimo estado e é constante;

$\Omega(t)$ representa o sinal comum a todos os ratos e é determinístico;

$\Gamma_{m}(t)$ representa o sinal comum do m-ésimo estado e é determinístico com restrição

$$
\sum_{m=1}^{M} L_{m} \Gamma_{m}(t)=0 ;
$$

$\epsilon_{l m}(\cdot)$ são séries estacionárias, independentes, com média zero, função de covariância $c_{\epsilon \epsilon}(u)$ e espectro $f_{\epsilon \epsilon}(\lambda)$.

Para este modelo, temos

$$
\begin{gathered}
E Y_{l m}(t)=\mu_{l m}+\Omega(t)+\Gamma_{m}(t), \\
\operatorname{Cov}\left\{Y_{l m}(t+u), Y_{l m}(t)\right\}=c_{\epsilon \varepsilon}(u)
\end{gathered}
$$

e

$$
\operatorname{Cov}\left\{Y_{l m}(t+u), Y_{l^{\prime} m^{\prime}}(t)\right\}=0, \quad\left(l^{\prime}, m^{\prime}\right) \neq(l, m)
$$

Nosso problema é:

(1) detectar se existe diferença entre os efeitos dos tratamentos, para isso, podemos testar

$$
H_{03}: \Gamma_{1}(t)=\Gamma_{2}(t)=\cdots=\Gamma_{M}(t)=0
$$

para todo $\mathrm{t}$;

(2) verificar a existência de similaridade entre todas as séries, ou seja, testarmos

$$
H_{04}: \Omega(t)=0
$$


Se k é um inteiro com $\frac{2 \pi k}{T} \simeq \lambda \not \equiv 0$, o modelo (4.27) pode ser escrito em termos da transformada discreta de Fourier

$$
\begin{aligned}
Y_{l m}^{\wedge}(k) & =\Omega^{\wedge}(k)+\Gamma_{m}^{\wedge}(k)+\epsilon_{l m}^{\wedge}(k) \\
& =\Omega^{\wedge}(k)+\Gamma_{m}^{\wedge}(k)+\xi_{l m k}+O_{\text {a.s. }}(1)
\end{aligned}
$$

para $l=1, \cdots, L_{m}, m=1, \cdots, M, k=1, \cdots, K \operatorname{com} \xi_{l m k} \sim N^{c}\left(0, f_{\epsilon \epsilon}(\lambda)\right)$ independentes.

Para fazer a análise consideraremos as seguintes somas de quadrados:

$$
\begin{gathered}
S Q H_{04}(\lambda)=N \sum_{k}\left|Y_{. .}^{\wedge}(k)\right|^{2}, \\
S Q H_{03}(\lambda)=\sum_{k} \sum_{m} L_{m}\left|Y_{\cdot m}^{\wedge}(k)-Y_{. .}^{\wedge}(k)\right|^{2},
\end{gathered}
$$

e

$$
S Q R_{03}(\lambda)=\sum_{k} \sum_{m} \sum_{l}\left|Y_{l m}^{\wedge}(k)-Y_{\cdot m}^{\wedge}(k)\right|^{2}
$$

onde

$$
Y_{\cdot m}^{\wedge}(k)=L_{m}^{-1} \sum_{l} Y_{l m}^{\wedge}(k)
$$

$\mathrm{e}$

$$
Y_{. .}^{\wedge}(k)=N^{-1} \sum_{m} \sum_{l} Y_{l m}^{\wedge}(k)=N^{-1} \sum_{m} L_{m} Y_{\cdot m}^{\wedge}(k)
$$

Usando a expressão (4.31) e a extensão complexa do teorema de FisherCochran (ver Capítulo 2), as expressões (4.32), (4.33) e (4.34) convergem quase certamente para

$$
\begin{gathered}
f_{\epsilon \epsilon}(\lambda) \chi_{2 K}^{2}\left(\frac{N \sum_{k}\left|\Omega^{\wedge}(k)\right|^{2}}{f_{\epsilon \epsilon}(\lambda)}\right) / 2 \\
f_{\epsilon \epsilon}(\lambda) \chi_{2 K(M-1)}^{2}\left(\frac{\sum_{k} \sum_{m} L_{m}\left|\Gamma_{m}^{\wedge}(k)\right|^{2}}{f_{\epsilon \epsilon}(\lambda)}\right) / 2
\end{gathered}
$$

$\mathrm{e}$

$$
f_{\epsilon \epsilon}(\lambda) \chi_{2 K(N-M)}^{2} / 2,
$$

respectivamente, com $\chi_{\nu}^{2}$ independentes. 
Os resultados estão resumidos na Tabela 4.3.

O teste para (4.29) pode ser realizado utilizando a estatística

$$
F_{03}(\lambda)=\frac{2 S Q H_{03}(\lambda) / 2 K(M-1)}{2 S Q R_{03}(\lambda) / 2 K(N-M)}
$$

que tem uma distribuição F com parâmetro de não centralidade $\frac{\sum_{k} \sum_{m=1}^{M} L_{m}\left|\Gamma_{m}^{\wedge}(k)\right|^{2}}{f_{\epsilon \epsilon}(\lambda)}$. Sob $H_{03},(4.40)$ tem uma distribuição $\mathrm{F}(2 K(M-1), 2 K(N-M))$ para $0 \leq \lambda<\pi$.

O teste para (4.30) pode ser realizado utilizando a estatística

$$
F_{04}(\lambda)=\frac{2 S Q H_{04}(\lambda) / 2 K}{2 S Q R_{03}(\lambda) / 2 K(N-M)}
$$

que também tem uma distribuição $\mathrm{F}$ com parâmetro de não centralidade $\frac{N \sum_{k}\left|\Omega^{\wedge}(k)\right|^{2}}{f_{\epsilon \epsilon}(\lambda)}$. Sob $H_{04},(4.41)$ tem uma distribuição $\mathrm{F}(2 K, 2 K(N-M))$.

Maiores informações, ver Brillinger(1973, 1980).

Se quisermos usar os componentes espectrais não suavizados para testar (4.29) e (4.30), na frequência $\lambda_{k}=\frac{2 \pi k}{T}, k=0,1, \cdots, \frac{[T-1]}{2}$, utilizaremos as estatísticas

$$
F_{03}\left(\lambda_{k}\right)=\frac{2 \sum_{m=1}^{M} L_{m}\left|Y_{\cdot m}^{\wedge}(k)-Y_{. .}^{\wedge}(k)\right|^{2} / 2(M-1)}{2 \sum_{m=1}^{M} \sum_{l=1}^{L_{m}}\left|Y_{l m}^{\wedge}(k)-Y_{\cdot m}^{\wedge}(k)\right|^{2} / 2(N-M)}
$$

$\mathrm{e}$

$$
F_{04}\left(\lambda_{k}\right)=\frac{2 N\left|Y_{. .}^{\wedge}(k)\right|^{2} / 2}{2 \sum_{m=1}^{M} \sum_{l=1}^{L_{m}}\left|Y_{l m}^{\wedge}(k)-Y_{\cdot m}^{\wedge}(k)\right|^{2} / 2(N-M)},
$$

com parâmetros de não centralidade $\frac{\sum_{m} L_{m}\left|\Gamma_{m}^{\wedge}\left(\lambda_{k}\right)\right|^{2}}{f_{\epsilon \epsilon}\left(\lambda_{k}\right)}$ e $\frac{N\left|\Omega^{\wedge}(k)\right|^{2}}{f_{\epsilon \epsilon}(\lambda)}$, que sob $H_{03}$ e $H_{04}$, têm distribuições $\mathrm{F}(2(M-1), 2(N-M))$ e $\mathrm{F}(2,2(N-M))$, respectivamente.

O efeito do tratamento $\Gamma_{m}^{\wedge}(k)$ pode ser estimado por

$$
Y_{\cdot m}^{\wedge}(k)-Y_{. .}^{\wedge}(k)
$$


com as seguintes propriedades

$$
\begin{gathered}
E\left[Y_{\cdot m}^{\wedge}(k)-Y_{. .}^{\wedge}(k)\right]=\Gamma_{m}^{\wedge}(k) \\
\operatorname{Var}\left[Y_{\cdot m}^{\wedge}(k)-Y_{. .}^{\wedge}(k)\right]=\frac{L_{m}-1}{L_{m}} f_{\epsilon \epsilon}(\lambda) .
\end{gathered}
$$

$\mathrm{e}$

Tabela 4.3: ANOVAF para os testes (4.29) e (4.30) na frequência $\lambda$

\begin{tabular}{|c|c|c|c|}
\hline fonte & $\begin{array}{l}\text { quadrado médio }=2 S Q / g l \\
(Q M)\end{array}$ & $\begin{array}{l}\text { estatística } \\
\qquad F\end{array}$ & $\begin{array}{c}\text { quadrado médio esperado } \\
\qquad(E Q M)\end{array}$ \\
\hline$\Omega(t)$ & $\frac{N \sum_{k}|Y \wedge(k)|^{2}}{K}(*)$ & $(*) /(* * *)$ & $f_{\epsilon \epsilon}(\lambda)+N f_{\Omega \Omega}^{(T)}(\lambda)$ \\
\hline$\Gamma_{m}(t)$ & $\frac{\sum_{k} \sum_{m=1}^{M} L_{m}\left|Y_{. m}^{\wedge}(k)-Y_{. .}^{\wedge}(k)\right|^{2}}{K(M-1)}(* *)$ & $(* *) /(* * *)$ & $f_{\epsilon \epsilon}(\lambda)+\frac{\sum_{m=1}^{M} L_{m} f_{\Gamma_{m} \Gamma_{m}}^{(T)}(\lambda)}{M-1}$ \\
\hline resíduo & $\sum_{k} \sum_{m=1}^{M} \frac{\sum_{l=1}^{L_{m}}\left|Y_{l m}^{\wedge}(k)-Y_{. m}^{\wedge}(k)\right|^{2}}{K(N-M)}(* * *)$ & & $f_{\epsilon \epsilon}(\lambda)$ \\
\hline
\end{tabular}

\begin{tabular}{lcc}
\hline \hline fonte & soma de quadrados & grau de liberdade \\
& $(S Q)$ & $(g l)$ \\
\hline$\Omega(t)$ & $N \sum_{k}\left|Y_{. .}(k)\right|^{2}$ & $2 K$ \\
$\Gamma_{m}(t)$ & $\sum_{k} \sum_{m=1}^{M} L_{m}\left|Y_{\cdot m}^{\wedge}(k)-Y_{. .}^{\wedge}(k)\right|^{2}$ & $2 K(M-1)$ \\
resíduo & $\sum_{k} \sum_{m=1}^{M} \sum_{l=1}^{L_{m}}\left|Y_{l m}^{\wedge}(k)-Y_{\cdot m}^{\wedge}(k)\right|^{2}$ & $2 K(N-M)$ \\
\hline Total & $\sum_{k} \sum_{m=1}^{M} \sum_{l=1}^{L_{m}}\left|Y_{l m}^{\wedge}(k)\right|^{2}$ & $2 K N$ \\
\hline \hline
\end{tabular}




\subsubsection{O modelo com fator aleatório}

Para exemplificar a utilização desse modelo, suponha que queremos verificar se existe diferença entre os componentes climáticos de estações americanas e européias, e também, se existe um componente climático comum para os dois continentes. Para fazer a análise, selecionamos aleatoriamente $L$ estações de cada um dos continentes, sendo $Y_{l m}(t)$ as temperaturas mensais da l-ésima estação no m-ésimo continente. Este exemplo é citado por Brillinger(1973). Neste caso, podemos modelar os dados utilizando (4.27),

$$
Y_{l m}(t)=\mu_{l m}+\Omega(t)+\Gamma_{m}(t)+\epsilon_{l m}(t)
$$

onde

$\mu_{l m}$ é a média da temperatura mensal da l-ésima estação no m-ésimo continente e é constante;

$\Omega(\cdot)$ representa o componente climático comum a todas as estações e é uma série estacionária com média zero, função de covariância $c_{\Omega \Omega}(u)$ e espectro $f_{\Omega \Omega}(\lambda)$;

$\Gamma_{m}(\cdot)$ representa o componente climático comum do m-ésimo continente e é uma série estacionária com média zero, função de covariância $c_{\Gamma \Gamma}(u)$ e espectro comum $f_{\Gamma \Gamma}(\lambda)$;

$\Omega(\cdot), \Gamma_{m}(\cdot)$ e $\epsilon_{l m}(\cdot)$ são independentes.

Sob as suposições do modelo, temos

$$
\begin{gathered}
E\left(Y_{l m}(t)\right)=\mu_{l m}, \\
\operatorname{Cov}\left\{Y_{l m}(t+u), Y_{l m}(t)\right\}=c_{\Omega \Omega}(u)+c_{\Gamma \Gamma}(u)+c_{\epsilon \epsilon}(u), \\
\operatorname{Cov}\left\{Y_{l m}(t+u), Y_{l^{\prime} m}(t)\right\}=c_{\Omega \Omega}(u)+c_{\Gamma \Gamma}(u), \quad l \neq l^{\prime}
\end{gathered}
$$

$\mathrm{e}$

$$
\operatorname{Cov}\left\{Y_{l m}(t+u), Y_{l^{\prime}}(t)\right\}=c_{\Omega \Omega}(u), \quad m \neq m^{\prime}
$$

Neste caso, a representação (4.31) pode ser reescrita

$$
Y_{l m}^{\wedge}(k)=\gamma_{k}+\nu_{m k}+\xi_{l m k}+O_{a . s .}(1)
$$

onde 
(i) $\gamma_{k}$ é uma variável $N^{c}\left(0, f_{\Omega \Omega}(\lambda)\right)$;

(ii) $\nu_{m k}$ são variáveis $N^{c}\left(0, f_{\Gamma \Gamma}(\lambda)\right)$ independentes;

(iii) $\xi_{l m k}$ são variáveirs $N^{c}\left(0, f_{\epsilon \epsilon}(\lambda)\right)$ independentes;

(iv) $\gamma_{k}, \nu_{m k}$ e $\xi_{l m k}$ são independentes.

No caso de modelo balanceado, ou seja, $L_{m}=L$, para $m=1, \cdots, M$, utilizando a expressão (4.45) e a extensão complexa do teorema de FisherCochran (ver Capítulo 2), a soma de quadrados (4.34) continua valendo e as somas de quadrados (4.32) e (4.33) convergem quase certamente para

$$
\left[f_{\epsilon \epsilon}(\lambda)+L f_{\Gamma \Gamma}(\lambda)+L M f_{\Omega \Omega}(\lambda)\right] \chi_{2 K}^{2} / 2
$$

$\mathrm{e}$

$$
\left[f_{\epsilon \epsilon}(\lambda)+L f_{\Gamma \Gamma}(\lambda)\right] \chi_{2 K(M-1)}^{2} / 2,
$$

respectivamente, com $\chi_{\nu}^{2}$ independentes (Brillinger, 1973, 1980).

As hipóteses equivalentes a (4.29) e (4.30) são

$$
H_{05}: f_{\Gamma \Gamma}(\lambda)=0
$$

e

$$
H_{06}: f_{\Omega \Omega}(\lambda)=0
$$

respectivamente para $0 \leq \lambda<\pi$.

Neste caso, os resultados estão resumidos na Tabela 4.4.

A estatística para a realização do teste para (4.48) tem, sob $H_{05}$, uma distribuição $\mathrm{F}(2 K(M-1), 2 K(N-M))$. Se a hipótese $H_{05}$ é verdadeira, ou seja, $f_{\Gamma \Gamma}(\lambda)=0$, então o teste para (4.49) pode ser feito utilizando uma estatística $\mathrm{F}(2 K, 2 K(N-M))$.

Pela Tabela 4.4, um estimador de $f_{\Omega \Omega}(\lambda)$ pode ser obtido por

$$
f_{\Omega \Omega}^{(T)}(\lambda)=\left\{\frac{\sum_{k}\left|Y_{. .}^{\wedge}(k)\right|^{2}}{K}-\frac{\sum_{k} \sum_{m=1}^{M} L_{m}\left|Y_{. m}^{\wedge}(k)-Y_{. .}^{\wedge}(k)\right|^{2}}{K N(M-1)}\right\},
$$


com

$$
E\left\{f_{\Omega \Omega}^{(T)}(\lambda)\right\}=f_{\Omega \Omega}(\lambda) .
$$

Além disso, também podemos obter um estimador de $f_{\Gamma \Gamma}(\lambda)$,

$$
f_{\Gamma \Gamma}^{(T)}(\lambda)=\left\{\frac{\sum_{k} \sum_{m=1}^{M} L_{m}\left|Y_{\cdot m}^{\wedge}(k)-Y_{. .}^{\wedge}(k)\right|^{2}}{K(N-L)}-\frac{\sum_{k} \sum_{m=1}^{M} \sum_{l=1}^{L_{m}}\left|Y_{l m}^{\wedge}(k)-Y_{\cdot m}^{\wedge}(k)\right|^{2}}{K N(L-1)}\right\},
$$

com

$$
E\left\{f_{\Gamma \Gamma}^{(T)}(\lambda)\right\}=f_{\Gamma \Gamma}(\lambda) .
$$

\begin{tabular}{|c|c|c|c|}
\hline fonte & $\begin{array}{c}\text { quadrado médio }=2 S Q / g l \\
(Q M)\end{array}$ & $\begin{array}{c}\text { estatística } \\
F \\
F\end{array}$ & $\begin{array}{l}\text { quadrado médio esperado } \\
\qquad(E Q M)\end{array}$ \\
\hline$\Omega(t)$ & $\frac{N \sum_{k}|Y \wedge(k)|^{2}}{K}(*)$ & $(*) /(* * *)$ & $f_{\epsilon \epsilon}(\lambda)+L f_{\Gamma \Gamma}(\lambda)+L M f_{\Omega \Omega}(\lambda)$ \\
\hline$\Gamma_{m}(t)$ & $\frac{\sum_{k} \sum_{m=1}^{M} L_{m}\left|Y_{\cdot m}^{\wedge}(k)-Y_{.}^{\wedge}(k)\right|^{2}}{K(M-1)}(* *)$ & $(* *) /(* * *)$ & $f_{\epsilon \epsilon}(\lambda)+L f_{\Gamma \Gamma}(\lambda)$ \\
\hline resíduo & $\frac{\sum_{k} \sum_{m=1}^{M} \sum_{l l=1}^{L_{m}}\left|Y_{l m}(k)-Y_{m}(k)\right|^{2}}{K(N-M)}(* * *)$ & & $f_{\epsilon \epsilon}(\lambda)$ \\
\hline
\end{tabular}

Tabela 4.4: ANOVAF para os testes (4.48) e (4.49) na frequência $\lambda$

\begin{tabular}{lcc}
\hline \hline fonte & soma de quadrados & grau de liberdade \\
& $(S Q)$ & $(g l)$ \\
\hline$\Omega(t)$ & $N \sum_{k}\left|Y_{. .}^{\wedge}(k)\right|^{2}$ & $2 K$ \\
$\Gamma_{m}(t)$ & $\sum_{k} \sum_{m=1}^{M} L_{m}\left|Y_{\cdot m}^{\wedge}(k)-Y_{. .}^{\wedge}(k)\right|^{2}$ & $2 K(M-1)$ \\
resíduo & $\sum_{k} \sum_{m=1}^{M} \sum_{l=1}^{L_{m}}\left|Y_{l m}^{\wedge}(k)-Y_{\cdot m}^{\wedge}(k)\right|^{2}$ & $2 K(N-M)$ \\
\hline Total & $\sum_{k} \sum_{m=1}^{M} \sum_{l=1}^{L_{m}}\left|Y_{l m}^{\wedge}(k)\right|^{2}$ & $2 K N$ \\
\hline \hline
\end{tabular}

A análise do modelo não balanceado é realizada utilizando a analogia feita na ANOVA usual; ver Scheffé(1959) ou Searle(1971). 


\subsection{Modelo linear geral com matriz de pla- nejamento fixa (modelo de planejamen- to)}

O modelo (4.1) pode ser reparametrizado nos seguintes modelos.

\subsubsection{O modelo com efeito fixo}

Neste caso, para $t=0,1, \cdots, T-1$, consideraremos o modelo

$$
Y(t)=\mu+\sum_{u} X(t-u) \theta(u)+\epsilon(t)
$$

que pode ser particionado da seguinte forma

$$
Y(t)=\mu+\sum_{u} X_{1}(t-u) \theta_{1}(u)+\sum_{u} X_{2}(t-u) \theta_{2}(u)+\epsilon(t)
$$

onde

$Y(t)$ : vetor observável, de dimensão $N \times 1$;

$\mu$ : vetor constante, de dimensão $N \times 1$;

$X_{1}(t), X_{2}(t)$ : matrizes fixas e observáveis, de dimensão $N \times P_{1}$ e $N \times P_{2}$, respectivamente, e satisfazendo

$$
\sum\left|X_{1}(t)\right|<\infty, \quad \sum\left|X_{2}(t)\right|<\infty
$$

$\theta_{1}(u), \theta_{2}(u)$ : vetores determinísticos, de dimensão $P_{1} \times 1$ e $P_{2} \times 1$, respectivamente;

$\epsilon(t)$ : vetor de séries temporais estacionárias de dimensão $N \times 1$, com média zero, matriz espectral $f_{\epsilon \epsilon}(\lambda)$ e satisfazendo (2.22).

Podemos notar que o modelo com um sinal comum, (4.9), é um caso particular do (4.51) com

$$
P_{1}=0, \quad P_{2}=1, \quad \theta_{2}(u)=\Omega(u) \text { fixo para } u=0, \cdots, T-1
$$


e

$$
X_{2}(t-u)=\left[\begin{array}{c}
1 \\
\vdots \\
1
\end{array}\right] .
$$

No caso balanceado, o modelo com um fator, (4.27), também é um caso particular do (4.51) com

$$
\begin{gathered}
P_{1}=1, \quad \theta_{1}(u)=\Omega(u), \\
P_{2}=M, \quad \theta_{2}(u)=\left(\Gamma_{1}(u), \cdots, \Gamma_{M}(u)\right)^{\prime} \text { fixo, } \\
X_{1}(t-u)=\left[\begin{array}{ccc}
1 & \cdots & 0 \\
& \vdots & \\
1 & \cdots & 0 \\
& \ddots & \\
0 & \cdots & 1 \\
& \vdots & \\
0 & \cdots & 1
\end{array}\right] .
\end{gathered}
$$

Além disso, existem dois importantes casos particulares de (4.51):

(1) O modelo de planejamento com dois fatores fixos cruzados,

$$
Y_{l m q}(t)=\mu_{l m q}+\Omega(t)+\Gamma_{m}(t)+\Delta_{q}(t)+(\Gamma \Delta)_{m q}(t)+\epsilon_{l m q}(t),
$$

para $l=1, \cdots, L, m=1, \cdots, M, q=1, \cdots, Q$ e $N=L M Q$, onde

$L$ : número de séries submetidas ao m-ésimo nível do fator 1 e q-ésimo nível do fator 2 ;

$M$ : número de níveis do fator 1 ;

$Q$ : número de níveis do fator 2 ;

$\Gamma_{m}(t)$ : efeito do m-ésimo nível do fator 1 , com restrição $\sum_{m} \Gamma_{m}(t)=0$;

$\Delta_{q}(t)$ : efeito do q-ésimo nível do fator 2, com restrição $\sum_{q} \Delta_{q}(t)=0$;

$(\Gamma \Delta)_{m q}(t)$ : interação entre m-ésimo nível do fator 1 e q-ésimo nível do fator 2, com restrições $\sum_{m}(\Gamma \Delta)_{m q}(t)=0$ e $\sum_{q}(\Gamma \Delta)_{m q}(t)=0$;

$\Omega(t)$ : sinal comum entre todas as séries observadas.

Neste caso, nosso problema é: 
(a) verificar a existência de interação entre os dois fatores, para isso, podemos testar

$H_{07}:(\Gamma \Delta)_{11}(t)=\cdots=(\Gamma \Delta)_{M 1}(t)=(\Gamma \Delta)_{12}(t)=\cdots=(\Gamma \Delta)_{M Q}(t)=0$

para todo $t$;

(b) detectar se existe diferença entre os efeitos dos níveis do fator 1, ou seja, testarmos

$$
H_{08}: \Gamma_{1}(t)=\cdots=\Gamma_{M}(t)=0
$$

(c) detectar se existe diferença entre os efeitos dos níveis do fator 2 , ou seja,

$$
H_{09}: \Delta_{1}(t)=\cdots=\Delta_{Q}(t)=0 .
$$

Assim, podemos notar que

$$
\begin{aligned}
& P_{1}=1+M+Q, \quad \theta_{1}(u)=\left(\Omega(u), \Gamma_{1}(u), \cdots, \Gamma_{M}(u), \Delta_{1}(u), \cdots, \Delta_{Q}(u)\right)^{\prime}, \\
& P_{2}=M Q, \quad \theta_{2}(u)=\left((\Gamma \Delta)_{11}(u), \cdots,(\Gamma \Delta)_{M 1}(u),(\Gamma \Delta)_{12}(u), \cdots,(\Gamma \Delta)_{M Q}(u)\right)^{\prime}, \\
& X_{1}(t-u)=\left[\begin{array}{ccccccc}
1 & 1 & \cdots & 0 & 1 & \cdots & 0 \\
& & \vdots & & & \vdots & \\
1 & 1 & \cdots & 0 & 1 & \cdots & 0 \\
& & \ddots & & & \vdots & \\
1 & 0 & \cdots & 1 & 1 & \cdots & 0 \\
& & \vdots & & & \vdots & \\
1 & 0 & \cdots & 1 & 1 & \cdots & 0 \\
& & \vdots & & & \ddots & \\
1 & 1 & \cdots & 0 & 0 & \cdots & 1 \\
& & \vdots & & & \vdots & \\
1 & 1 & \cdots & 0 & 0 & \cdots & 1 \\
& & \ddots & & & \vdots & \\
1 & 0 & \cdots & 1 & 0 & \cdots & 1 \\
1 & 0 & \cdots & 1 & 0 & \cdots & 1
\end{array}\right] e X_{2}(t-u)=\left[\begin{array}{ccc}
1 & \cdots & 0 \\
\vdots & \\
1 & \cdots & 0 \\
& \ddots & \\
0 & \cdots & 1 \\
\vdots & \\
0 & \cdots & 1
\end{array}\right] .
\end{aligned}
$$


(2) O modelo de planejamento com dois fatores fixos hierárquicos

$$
Y_{l q m}(t)=\mu_{l m q}+\Omega(t)+\Gamma_{m}(t)+\Delta_{q(m)}(t)+\epsilon_{l(m q)}(t),
$$

para $l=1, \cdots, L, m=1, \cdots, M, q=1, \cdots, Q$ e $N=L M Q$, onde

$L$ : número de séries submetidas ao q-ésimo nível do fator 2 dentro do m-ésimo nível do fator 1 ;

$M$ : número de níveis do fator 1 ;

$Q:$ número de níveis do fator 2 dentro do fator 1 ;

$\Gamma_{m}(t)$ : efeito do m-ésimo nível do fator 1, com restrição $\sum_{m} \Gamma_{m}(t)=0$;

$\Delta_{q(m)}(t)$ : efeito do q-ésimo nível do fator 2 dentro do m-ésimo nível do fator 1 , com restrição $\sum_{q} \Delta_{q(m)}(t)=0$ para todo $m$;

$\Omega(t)$ : sinal comum entre todas as séries observadas.

Neste caso, nosso problema é

(a) detectar se existe diferença entre os efeitos dos níveis do fator 1 , ou seja, testarmos

$$
H_{10}: \Gamma_{1}(t)=\cdots=\Gamma_{M}(t)=0 ;
$$

(b) detectar se existe diferença entre os efeitos dos níveis do fator 2 dentro do m-ésimo nível do fator 1 , para isso, podemos testar

$$
H_{11}: \Delta_{1(m)}(t)=\cdots=\Delta_{Q(m)}(t)=0,
$$

para $m=1, \cdots, M$.

Aqui,

$$
\begin{gathered}
P_{1}=1+M Q, \theta_{1}(u)=\left(\Omega(u), \Delta_{1(1)}(u), \cdots, \Delta_{Q(1)}(u), \Delta_{1(2)}(u), \cdots, \Delta_{Q(M)}(u)\right)^{\prime} \\
P_{2}=M, \theta_{2}(u)=\left(\Gamma_{1}(u), \cdots, \Gamma_{M}(u)\right)^{\prime}, \\
X_{1}(t-u)=\left[\begin{array}{cccc}
1 & 1 & \cdots & 0 \\
& & \vdots & \\
1 & 1 & \cdots & 0 \\
& & \ddots & \\
1 & 0 & \cdots & 1 \\
& & \vdots & \\
1 & 0 & \cdots & 1
\end{array}\right] \text { e } X_{2}(t-u)=\left[\begin{array}{ccc}
1 & \cdots & 0 \\
& \vdots & \\
1 & \cdots & 0 \\
& \ddots & \\
0 & \cdots & 1 \\
& \vdots & \\
0 & \cdots & 1
\end{array}\right] .
\end{gathered}
$$


Para verificar se algum subconjunto dos parâmetros do modelo (4.51) está contribuindo significativamente para a resposta, testaremos a hipótese

$$
H_{12}: \theta_{2}(u)=0 \text {. }
$$

Para o modelo (4.9), este é essencialmente um problema de detectar um sinal comum (hipótese (4.10)); para o modelo (4.27), é de detectar a diferença entre os efeitos dos tratamentos (hipótese (4.29)); para o modelo (4.52), é de detectar a interação entre os dois fatores (hipótese (4.53)) e, para o modelo (4.56), é de detectar a diferença entre os efeitos dos níveis do fator 1 (hipótese (4.57)).

Um procedimento para fazer o teste (4.59) é comparar o modelo completo (4.51) com o modelo sob a hipótese $H_{12}$ (modelo reduzido),

$$
Y(t)=\sum_{u} X_{1}(t-u) \theta_{1}(u)+\epsilon(t) .
$$

Sob as condições do modelo, (4.51) pode ser representado em termos da transformada discreta de Fourier

$$
Y^{\wedge}(k)=X_{1}^{\wedge}(k) \theta_{1}^{\wedge}(k)+X_{2}^{\wedge}(k) \theta_{2}^{\wedge}(k)+\xi_{k}+O_{a . s .}(1)
$$

para $k=1, \cdots, K$, onde $\frac{2 \pi k}{T} \simeq \lambda, \frac{2 \pi k}{T} \neq 0, \pi \operatorname{com} \xi_{k} \sim N^{c}\left(0, f_{\epsilon \epsilon}\right)$ independentes.

Analogamente, (4.50) e (4.60) podem ser escritos como

$$
Y^{\wedge}(k)=X^{\wedge}(k) \theta^{\wedge}(k)+\xi_{k}+O_{a . s .}(1)
$$

$\mathrm{e}$

$$
Y^{\wedge}(k)=X_{1}^{\wedge}(k) \theta_{1}^{\wedge}(k)+\xi_{k}+O_{a . s .}(1)
$$

respectivamente.

Para fazer a análise consideraremos as seguintes somas de quadrados

$$
S Q H_{c}(\lambda)=\sum_{k}{\overline{Y^{\wedge}(k)}}^{T} X^{\wedge}(k)\left({\overline{X^{\wedge}(k)}}^{T} X^{\wedge}(k)\right)^{-1}{\overline{X^{\wedge}(k)}}^{T} Y^{\wedge}(k),
$$




$$
S Q H_{12}(\lambda)=\sum_{k}{\overline{Y^{\wedge}(k)}}^{T} X_{1}^{\wedge}(k)\left({\overline{X_{1}^{\wedge}(k)}}^{T} X_{1}^{\wedge}(k)\right)^{-1}{\overline{X_{1}^{\wedge}(k)}}^{T} Y^{\wedge}(k)
$$

$\mathrm{e}$

$S Q R_{c}(\lambda)=\sum_{k}\left\{{\overline{Y^{\wedge}(k)}}^{T} Y^{\wedge}(k)-{\overline{Y^{\wedge}(k)}}^{T} X^{\wedge}(k)\left({\overline{X^{\wedge}}(\bar{k})}^{T} X^{\wedge}(k)\right)^{-1}{\overline{X^{\wedge}(k)}}^{T} Y^{\wedge}(k)\right\}$

onde

$$
X^{\wedge}(k)=\left[\begin{array}{ll}
X_{1}^{\wedge}(k) & X_{2}^{\wedge}(k)
\end{array}\right] .
$$

Os resultados estão resumidos nas Tabelas 4.5 e 4.6 , onde

(i) $e^{\wedge}(k)=Y^{\wedge}(k)-X^{\wedge}(k) \theta^{\wedge}(k)$;

(ii) $A^{\wedge}(k)=X^{\wedge}(k) \theta^{\wedge}(k)$;

(iii) $B^{\wedge}(k)=X_{1}^{\wedge}(k) \theta_{1}^{\wedge}(k)$.

O teste para (4.59) pode ser realizado utilizando a estatistica

$$
F_{12}(\lambda)=\frac{\left(S Q H_{c}(\lambda)-S Q H_{12}(\lambda)\right) / 2 K P_{2}}{S Q R_{c}(\lambda) / 2 K(N-P)}
$$

que, sob $H_{12}$, tem uma distribuição $\mathrm{F}\left(2 K P_{2}, 2 K(N-P)\right)$.

Para mais detalhes, ver Brillinger(1980).

Se quisermos usar os componentes espectrais não suavizados para testar (4.59), na frequência $\lambda_{k}=\frac{2 \pi k}{T}, k=0,1, \cdots, \frac{[T-1]}{2}$, utilizaremos a estatistica

$$
F_{12}\left(\lambda_{k}\right)=\frac{\left(S Q H_{c}\left(\lambda_{k}\right)-S Q H_{12}\left(\lambda_{k}\right)\right) / 2 P_{2}}{S Q R_{c}\left(\lambda_{k}\right) / 2(N-P)}
$$

que, sob $H_{12}$, tem uma distribuição $\mathrm{F}\left(2 P_{2}, 2(N-P)\right)$, onde

$$
\begin{aligned}
& S Q H_{c}\left(\lambda_{k}\right)={\overline{Y^{\wedge}(k)}}^{T} X^{\wedge}(k)\left({\overline{X^{\wedge}(k)}}^{T} X^{\wedge}(k)\right)^{-1}{\overline{X^{\wedge}(k)}}^{T} Y^{\wedge}(k), \\
& S Q H_{12}\left(\lambda_{k}\right)={\overline{Y^{\wedge}(k)}}^{T} X_{1}^{\wedge}(k)\left({\overline{X_{1}^{\wedge}(k)}}^{T} X_{1}^{\wedge}(k)\right)^{-1}{\overline{X_{1}^{\wedge}(k)}}^{T} Y^{\wedge}(k)
\end{aligned}
$$

$\mathrm{e}$

$$
S Q R_{c}\left(\lambda_{k}\right)={\overline{Y^{\wedge}(k)}}^{T} Y^{\wedge}(k)-{\overline{Y^{\wedge}(k)}}^{T} X^{\wedge}(k)\left({\overline{X^{\wedge}(k)}}^{T} X^{\wedge}(k)\right)^{-1}{\overline{X^{\wedge}(k)}}^{T} Y^{\wedge}(k) .
$$


Tabela 4.5: ANOVAF para o teste (4.59) na frequência $\lambda$

\begin{tabular}{ll}
\hline \hline fonte & \multicolumn{1}{c}{ soma de quadrados } \\
& \multicolumn{1}{c}{$(S Q)$} \\
\hline Modelo reduzido & $\sum_{k}{\overline{Y^{\wedge}(k)}}^{T} X_{1}^{\wedge}(k)\left({\overline{X_{1}^{\wedge}(k)}}^{T} X_{1}^{\wedge}(k)\right)^{-1}{\overline{X_{1}^{\wedge}(k)}}^{T} Y^{\wedge}(k)(*)$ \\
Modelo só com $\theta_{2}(t)$ & $(* *)-(*)$ \\
\hline Modelo completo & $\sum_{k}{\overline{Y^{\wedge}(k)}}^{T} X^{\wedge}(k)\left({\overline{X^{\wedge}(k)}}^{T} X^{\wedge}(k)\right)^{-1}{\overline{X^{\wedge}(k)}}^{T} Y^{\wedge}(k)(* *)$ \\
resíduo & $(* * *)-(* *)$ \\
\hline Total & $\sum_{k}{\overline{Y^{\wedge}(k)}}^{T} Y^{\wedge}(k)(* * *)$ \\
\hline \hline
\end{tabular}

\begin{tabular}{lccc}
\hline \hline fonte & grau de liberdade & quadrado médio $=\frac{2 S Q}{g l}$ & estatística \\
& $(g l)$ & $(Q M)$ & $F$ \\
\hline Modelo reduzido & $2 K P_{1}$ & $\sum_{n=1}^{N} f_{B_{n} B_{n}}^{(T)}(\lambda) / P_{1}$ & \\
Modelo só com $\theta_{2}(t)$ & $2 K P_{2}$ & & $\frac{((* *)-(*)) / 2 K P_{2}}{((* * *)-(* *)) / 2 K(N-P)}$ \\
\hline Modelo completo & $2 K P$ & $\sum_{n=1}^{N} f_{A_{n} A_{n}}^{(T)}(\lambda) / P$ & $\frac{(* *) / 2 K P}{((* * *)-(* *)) / 2 K(N-P)}$ \\
resíduo & $2 K(N-P)$ & $\sum_{n=1}^{N} f_{e_{n} e_{n}}^{(T)}(\lambda) /(N-P)$ & \\
\hline Total & $2 K N$ & & \\
\hline \hline
\end{tabular}

Um estimador de $\theta^{\wedge}(k)$ é

$$
\hat{\theta}^{\wedge}(k)=\left({\overline{X^{\wedge}(k)}}^{T} X^{\wedge}(k)\right)^{-1}{\overline{X^{\wedge}(k)}}^{T} Y^{\wedge}(k),
$$

se $\left({\overline{X^{\wedge}(k)}}^{T} X^{\wedge}(k)\right)^{-1}$ existe, com as seguintes propriedades

$$
E\left[\hat{\theta}^{\wedge}(k)\right]=\theta^{\wedge}(k)
$$

e

$$
\operatorname{Var}\left[\hat{\theta}^{\wedge}(k)\right]=\left({\overline{X^{\wedge}(k)}}^{T} X^{\wedge}(k)\right)^{-1} f_{\epsilon \epsilon}(\lambda) .
$$


No caso $\theta_{2}(u)=0$, um estimador de $\theta_{1}^{\wedge}(k)$ pode ser

$$
\hat{\theta}_{1}^{\wedge}(k)=\left({\overline{X_{1}^{\wedge}(k)}}^{T} X_{1}^{\wedge}(k)\right)^{-1}{\overline{X_{1}^{\wedge}(k)}}^{T} Y^{\wedge}(k) .
$$

Para mais detalhes, ver Roy et al(1971) e Brillinger(1980).

Tabela 4.6: Quadrado médio esperado na frequência $\lambda$ no caso $\theta_{2}(u)=0$

\begin{tabular}{ll}
\hline \hline fonte & quadrado médio esperado \\
& \multicolumn{1}{c}{$(E Q M)$} \\
\hline Modelo reduzido & $f_{\epsilon \epsilon}(\lambda)+\frac{\operatorname{tr}\left\{f_{\theta_{1} \theta_{1}}^{(T)}(\lambda) \overline{X_{1}(\lambda)^{T}} X_{1}(\lambda)\right\}_{a}}{P_{1}}$ \\
Modelo só com $\theta_{2}(t)$ & $f_{\epsilon \epsilon}(\lambda)$ \\
\hline Modelo completo & $f_{\epsilon \epsilon}(\lambda)+\frac{\left.\operatorname{tr}\left\{f_{\theta \theta}^{(T)}(\lambda) \overline{X(\lambda)}\right)^{T} X(\lambda)\right\}}{P}$ \\
Resíduo & $f_{\epsilon \epsilon}(\lambda)$ \\
\hline \hline & ${ }^{a} X(\lambda)=\sum_{u} \exp \{-i \lambda u\} X(u)$
\end{tabular}

Um teste análogo do (4.59) é testar a hipótese

$$
H_{13}: \theta(u)=0,
$$

que pode ser feita da seguinte forma

(1) utilizar os componentes espectrais suavizados, com $K$ frequências em torno de $\lambda$,

$$
F_{13}(\lambda)=\frac{S Q H_{c}(\lambda) / 2 K P}{S Q R_{c}(\lambda) / 2 K(N-P)}
$$

que, sob $H_{13}$, tem uma distribuição $\mathrm{F}(2 K P, 2 K(N-P))$;

(2) utilizar os componentes não suavizados, na frequência $\lambda_{k}=\frac{2 \pi k}{T}, k=$ $0,1, \cdots, \frac{[T-1]}{2}$,

$$
F_{13}\left(\lambda_{k}\right)=\frac{S Q H_{c}\left(\lambda_{k}\right) / 2 P}{S Q R_{c}\left(\lambda_{k}\right) / 2(N-P)}
$$

que, sob $H_{13}$, tem uma distribuição $\mathrm{F}(2 P, 2(N-P))$ (Shumway, 1988). 


\subsubsection{O modelo com efeito aleatório}

Consideremos o modelo (4.51),

$$
Y(t)=\mu+\sum_{u} X_{1}(t-u) \theta_{1}(u)+\sum_{u} X_{2}(t-u) \theta_{2}(u)+\epsilon(t),
$$

onde

$Y(t)$ : vetor observável, de dimensão $N \times 1$;

$\mu$ : vetor constante, de dimensão $N \times 1$;

$X_{1}(t), X_{2}(t)$ : matrizes fixas e observáveis, de dimensão $N \times P_{1}$ e $N \times P_{2}$, respectivamente, e satisfazendo

$$
\sum\left|X_{1}(t)\right|<\infty, \quad \sum\left|X_{2}(t)\right|<\infty
$$

$\theta_{1}(u), \theta_{2}(u)$ : séries estacionárias, independentes com média zero, matrizes espectrais $f_{\theta_{1} \theta_{1}}(\lambda)$ e $f_{\theta_{2} \theta_{2}}(\lambda)$, respectivamente, e satisfazendo (2.22);

$\epsilon(t)$ : vetor de séries temporais estacionárias de dimensão $N \times \dot{1}$, com média zero, matriz espectral $f_{\epsilon \epsilon}(\lambda)$ e satisfazendo (2.22).

Neste caso, a representação (4.61) pode ser reescrita

$$
\begin{aligned}
Y^{\wedge}(k) & =X_{1}^{\wedge}(k) \theta_{1}^{\wedge}(k)+X_{2}^{\wedge}(k) \theta_{2}^{\wedge}(k)+\xi_{k}+O_{a . s .} \\
& =X_{1}^{\wedge}(k) \Theta_{1 k}+X_{2}^{\wedge}(k) \Theta_{2 k}+\xi_{k}+O_{a . s .}
\end{aligned}
$$

onde

(i) $\Theta_{1 k}, \Theta_{2 k}$ : variáveis $N^{c}\left(0, f_{\theta_{1} \theta_{1}}(\lambda)\right)$ e $N^{c}\left(0, f_{\theta_{2} \theta_{2}}(\lambda)\right.$ independentes;

(ii) $\xi_{k}$ : variáveis $N^{c}\left(0, f_{\epsilon \epsilon}(\lambda)\right.$ independentes;

(iii) $\Theta_{1 k}, \Theta_{2 k}$ e $\xi_{k}$ : são independentes.

A hipótese equivalente a (4.59) é

$$
H_{14}: f_{\theta_{2} \theta_{2}}(\lambda)=0, \quad 0 \leq \lambda<\pi .
$$

Analogamente, a hipótese equivalente a (4.72) é

$$
H_{15}: f_{\theta \theta}(\lambda)=0, \quad 0 \leq \lambda<\pi
$$


Neste caso, os resultados apresentados na Tabela 4.5 continuam valendo, e os valores de quadrado médio esperado estão representados na Tabela 4.7.

Tabela 4.7: Quadrado médio esperado para os teses (4.76) e (4.77) na frequência $\lambda$

\begin{tabular}{ll}
\hline \hline fonte & \multicolumn{1}{c}{$\begin{array}{c}\text { quadrado médio esperado } \\
(E Q M)\end{array}$} \\
\hline Modelo reduzido & $(\star \star)-(\star)$ \\
Modelo só com $\theta_{2}(t)$ & $f_{\epsilon \epsilon}(\lambda)+\frac{\left.\operatorname{tr}\left\{f_{\theta_{2} \theta_{2}}(\lambda){\overline{X_{2}(\lambda)}}^{T}\left[I-X_{1}(\lambda)\left(\overline{X_{1}(\lambda)}\right)^{T} X_{1}(\lambda)\right)^{-1} \overline{X_{1}(\lambda)^{T}}\right] X_{2}(\lambda)\right\}}{P_{2}}(\star)$ \\
\hline Modelo completo & $f_{\epsilon \epsilon}(\lambda)+\frac{\operatorname{tr}\left\{f_{\theta \theta}(\lambda) \overline{X(\lambda)}^{T} X(\lambda)\right\}}{P}(\star \star)$ \\
Resíduo & $f_{\epsilon \epsilon}(\lambda)$ \\
\hline \hline
\end{tabular}

Podemos notar que o modelo com um sinal comum estocástico é um caso particular do (4.75) com $P_{1}=0, P_{2}=1$ e $\Theta_{2 k}=\gamma_{k}$. No caso balanceado, o modelo com um fator aleatório, também é um caso particular do (4.75) com $P_{1}=1, \Theta_{1 k}=\gamma_{k}, P_{2}=M$ e $\Theta_{2 k}=\left(\nu_{1 k}, \cdots, \nu_{M k}\right)^{\prime}$.

As análises feitas para o modelo com um sinal comum estocástico, (4.22), e o modelo com um fator aleatório, (4.45), são análogas às do modelo linear com matriz de planejamento fixa com efeito aleatório, (4.75). No caso de modelo balanceado, as hipóteses (4.76) e (4.77) podem ser testadas da mesma maneira que para o modelo com efeito fixo, isto é, utilizando as estatísticas (4.68)-(4.69) e (4.73)-(4.74), respectivamente. No caso não balanceado as estatísticas envolvidas nos testes têm distribuições assintóticas mais complicadas. Para melhores referências ver Scheffé(1959), Searle(1971), Brillinger(1980, 1985) e Shumway(1988). 


\subsection{Modelo linear geral com matriz de pla- nejamento aleatória (modelo de regres- são múltipla)}

O problema geral é relacionar uma coleção de $\mathrm{P}$ séries de entrada, tais como $X_{1}(t), X_{2}(t), \cdots, X_{P}(t)$, com uma série de saída $Y(t)$. Por exemplo, podemos considerar as séries de entrada como nível de fumaça, nível de dioxido sulfúrico, temperatura e humidade relativa, denotadas $X_{1}(t), X_{2}(t)$, $X_{3}(t)$ e $X_{4}(t)$, respectivemente, e uma série de saída, tal como mortalidade, denotada $Y(t)$. Assim, podemos modelar os dados como

$$
Y(t)=\sum_{u} X(t-u) \theta(u)+\epsilon(t)
$$

para $t=0,1, \cdots, T-1$, onde

$X^{\prime}(t)$ : vetor com $P$ componentes que são séries temporais estacionárias conhecidas, com média zero, matriz de covariância $c_{x x}(u)$ e matriz espectral $f_{x x}(\lambda)$.

$\theta(u)$ : vetor de coeficientes desconhecidos com $P$ componentes;

$\epsilon(t)$ : séries estacionárias, independentes com média zero, espectro $f_{\epsilon \epsilon}(\lambda)$ e não correlacionada com $X(t)$;

$X_{j}(t)$ e $Y(t)$ são processos conjuntamente estacionários, $j=1, \cdots, P$.

Estamos interessados em verificar se todas as séries de entrada no modelo estão conjuntamente influenciando a série de saída, ou somente algumas séries de entrada estão contribuíndo significativamente para a série de saída.

Para verificar se todas as séries estão conjuntamente influenciando a série de saída, testaremos

$$
H_{16}: \theta(u)=0
$$

Para fazer a análise, reescrevemos o modelo (4.78) em termos da transformada discreta de Fourier

$$
Y^{\wedge}(k)=X_{k}^{\wedge} \theta^{\wedge}(k)+\xi_{k}+O_{a . s .}(1)
$$

onde 
(i) $X_{k}^{\wedge}$ : variáveis $N^{c}\left(0, f_{x x}(\lambda)\right)$ independentes;

(ii) $\xi_{k}$ : variáveis $N^{c}\left(0, f_{\epsilon \epsilon}\right)$ independentes.

O teste para (4.79) pode ser realizado utilizando a estatística

$$
F_{16}(\lambda)=\frac{\bar{f}_{x y}^{(T)}(\lambda)\left(f_{x x}^{(T)}(\lambda)\right)^{-1} f_{x y}^{(T)}(\lambda) / 2 P}{f_{y x}^{(T)}(\lambda) / 2(K-P)},
$$

que sob $H_{16}$, tem uma distribuição $\mathrm{F}(2 P, 2(K-P))$ para $\frac{2 \pi k}{T} \simeq \lambda, k=$ $1, \cdots, K$ e $K>P$, onde

$$
\begin{gathered}
f_{x x}^{(T)}(\lambda)=K^{-1} \sum_{k} X_{k}^{\wedge} \bar{X}_{k}^{\wedge}, \\
f_{x y}^{(T)}(\lambda)=K^{-1} \sum_{k} X_{k}^{\wedge} \bar{Y}^{\wedge}(k), \\
f_{y y}^{(T)}(\lambda)=K^{-1} \sum_{k} Y^{\wedge}(k) \bar{Y}^{\wedge}(k)
\end{gathered}
$$

$\mathrm{e}$

$$
f_{y x}^{(T)}(\lambda)=f_{y y}^{(T)}(\lambda)-\bar{f}_{x y}^{(T)}(\lambda)\left(\bar{f}_{x x}^{(T)}(\lambda)\right)^{-1} f_{x y}^{(T)}(\lambda) .
$$

Ver Shumway $(1970,1988)$, Brillinger(1973,1980), Goodman(1963) e Wahba(1968).

Para verificar se somente algumas séries de entrada estão contribuíndo significativamente para o modelo, particionamos o vetor de entrada $X(t) \mathrm{em}$ $P_{1}$ e $P_{2}$ componentes, isto é, $X(t)=\left(X_{1}(t), X_{2}(t)\right)$ e $\theta(u)=\left(\theta_{1}(u), \theta_{2}(u)\right)^{\prime}$, reescrevendo o modelo (4.78) da seguinte maneira:

$$
Y(t)=\sum_{u} X_{1}(t-u) \theta_{1}(u)+\sum_{u} X_{2}(t-u) \theta_{2}(u)+\epsilon(t)
$$

Neste caso, a hipótese a ser testada é

$$
H_{17}: \theta_{2}(u)=0 .
$$

Para fazer a análise, consideraremos o modelo (4.86) sob $H_{17}$, que se reduz a

$$
Y(t)=\sum_{u} X_{1}(t-u) \theta_{1}(u)+\epsilon(t)
$$


e comparamos os modelos (4.86) e (4.88) em algumas frequências $\frac{2 \pi k}{T} \simeq \lambda$, $k=1, \cdots, K$, onde $K>P$. Isso equivale a testar se $Y^{\wedge}(k)$, dado por $X^{\wedge}(k)=\left(X_{1}^{\wedge}(k), X_{2}^{\wedge}(k)\right)$, tem uma distribuição normal complexa com

$$
E\left[Y^{\wedge}(k)\right]=X_{1}^{\wedge}(k) \theta_{1}^{\wedge}(k)+X_{2}^{\wedge}(k) \theta_{2}^{\wedge}(k) \text { e } \operatorname{Var}\left[Y^{\wedge}(k)\right]=f_{y x}(\lambda)
$$

ou

$$
E\left[Y^{\wedge}(k)\right]=X_{1}^{\wedge}(k) \theta_{1}^{\wedge}(k) \text { e } \operatorname{Var}\left[Y^{\wedge}(k)\right]=f_{y x}(\lambda) .
$$

As matrizes espectrais apresentadas em (4.82) e (4.83) podem ser particionadas

$$
f_{x x}^{(T)}=\left(\begin{array}{ll}
f_{11}^{(T)} & f_{12}^{(T)} \\
f_{21}^{(T)} & f_{22}^{(T)}
\end{array}\right)
$$

$\mathrm{e}$

$$
f_{x y}^{(T)}=\left(\begin{array}{c}
f_{1 y}^{(T)} \\
f_{2 y}^{(T)}
\end{array}\right)
$$

onde

- $f_{11}^{(T)}$ : matriz espectral de $X_{1}(t)$, de dimensão $P_{1}$ x $P_{1}$;

- $f_{22}^{(T)}$ : matriz espectral de $X_{2}(t)$, de dimensão $P_{2} \times P_{2}$;

- $f_{12}^{(T)}$ e $f_{21}^{(T)}$ : matrizes espectrais cruzadas de $X_{1}(t)$ com $X_{2}(t)$ e $X_{2}(t)$ com $X_{1}(t)$, de dimensões $P_{1} \times P_{2}$ e $P_{2} \times P_{1}$, respectivamente.

O teste para (4.87) é realizado utilizando a estatística

$$
F_{17}(\lambda)=\frac{\left(f_{y 1}^{(T)}(\lambda)-f_{y x}^{(T)}(\lambda)\right) / 2 P_{2}}{f_{y x}^{(T)}(\lambda) / 2(K-P)}
$$

que sob $H_{17}$, tem uma distribuição $\mathrm{F}\left(2 P_{2}, 2(K-P)\right)$, onde

$$
\begin{aligned}
& f_{y x}^{(T)}=f_{y y}^{(T)}-\bar{f}_{x y}^{(T)}\left(f_{x x}^{(T)}\right)^{-1} f_{x y}^{(T)} \\
& f_{y 1}^{(T)}=f_{y y}^{(T)}-\bar{f}_{1 y}^{(T)}\left(f_{11}^{(T)}\right)^{-1} f_{1 y}^{(T)} .
\end{aligned}
$$

Os resultados estão resumidos na Tabela 4.8 e Tabela 4.9 . 
Tabela 4.8: ANOVAF para os testes (4.79) e (4.87) na frequência $\lambda$

\begin{tabular}{llc}
\hline \hline fonte & \multicolumn{1}{c}{ soma de quadrados } & grau de liberdade \\
& \multicolumn{1}{c}{$(S Q)$} & $(g l)$ \\
\hline Modelo reduzido & $K \bar{f}_{1 y}^{(T)}(\lambda)\left(f_{11}^{(T)}(\lambda)\right)^{-1} f_{1 y}^{(T)}(\lambda)(*)$ & $2 P_{1}$ \\
Modelo só com $\theta_{2}(t)$ & $(* *)-(*)$ & $2 P_{2}$ \\
\hline Modelo completo & $K \bar{f}_{x y}^{(T)}(\lambda)\left(f_{x x}^{(T)}(\lambda)\right)^{-1} f_{x y}^{(T)}(\lambda)(* *)$ & $2 P$ \\
resíduo & $(* * *)-(* *)$ & $2(K-P)$ \\
\hline Total & $\mathrm{K} f_{y y}^{(T)}(\lambda)(* * *)$ & $2 K$ \\
\hline \hline
\end{tabular}

\begin{tabular}{lcc}
\hline \hline fonte & quadrado médio $=\frac{2 S Q}{g l}$ & estatística \\
& $(Q M)$ & $F$ \\
\hline Modelo reduzido & $\frac{(*)}{P_{1}}$ & \\
Modelo só com $\theta_{2}(t)$ & & $\frac{((* *)-(*)) / 2 P_{2}}{(* * *)-(* *)) / 2(K-P)}$ \\
\hline Modelo completo & $\frac{(* *)}{P}$ & $\frac{(* *) / 2 P}{((* * *)-(* *)) / 2(K-P)}$ \\
resíduo & $\frac{(* * *)-(* *)}{(K-P)}$ & \\
\hline Total & \\
\hline \hline
\end{tabular}

Para $k=1, \cdots, K$ e $\frac{2 \pi k}{T} \simeq \lambda$, um estimador para $\theta^{\wedge}(k)$ é

$$
\hat{\theta}^{\wedge}(k)=\bar{f}_{x y}^{(T)}(\lambda)\left(f_{x x}^{(T)}(\lambda)\right)^{-1}
$$

com as seguintes propriedades:

$$
E\left[\hat{\theta}^{\wedge}(k)\right]=\theta^{\wedge}(k)
$$

e

$$
\operatorname{Cov}\left(\hat{\theta}^{\wedge}(k)\right)=\frac{f_{y x}^{(T)}(\lambda)\left[\left(f_{x x}^{(T)}(\lambda)\right)^{\prime}\right]^{-1}}{K}
$$


Para detalhes ver Shumway $(1970,1988)$ e Brillinger(1973, 1980).

Tabela 4.9: Quadrado médio esperado para os testes (4.79) e (4.87) na frequência $\lambda$

\begin{tabular}{ll}
\hline \hline fonte & $\begin{array}{c}\text { quadrado médio esperado } \\
(E Q M)\end{array}$ \\
\hline Modelo reduzido & $f_{\epsilon \epsilon}(\lambda)+K \frac{\left\{f_{\theta_{1} \theta_{1}}^{(T)}(\lambda) f_{x x}(\lambda)\right\}}{P_{1}}$ \\
Modelo só com $\theta_{2}(t)$ & $f_{\epsilon \epsilon}(\lambda)$ \\
\hline Modelo completo & $f_{\epsilon \epsilon}(\lambda)+K \frac{\left\{f_{\theta \theta}^{(T)}(\lambda) f_{x x}(\lambda)\right\}}{P}$ \\
Resíduo & $f_{\epsilon \epsilon}(\lambda)$ \\
\hline \hline
\end{tabular}

\subsection{Modelo linear multivariado (modelo de regressão multivariado)}

Consideremos o modelo

$$
Y(t)=\mu+\sum_{u} \theta(u) X(t-u)+\epsilon(t)
$$

onde

$Y(t)$ : vetor observável com $N$ componentes que são séries temporais estacionárias;

$\mu$ : vetor constante com $N$ componentes;

$\theta(u)$ : função matricial fixa, de dimensão $N \times P$ e satisfazendo

$$
\sum_{u=-\infty}^{\infty}\{1+|u|\}|\theta(u)|<\infty
$$

$X(t)$ : função vetorial constante com $P$ componentes que são séries temporais estacionárias; 
$\epsilon(t)$ : vetor de séries temporais estacionárias de dimensão $N \times 1$, com média zero, matriz espectral $f_{\epsilon \epsilon}(\lambda)$ e satisfazendo (2.22).

Para exemplificar a utilização do modelo (4.93) considere $X(t)$ a série de manchas solares (ver Waldmeir, 1961), e $Y(t)$ temperaturas mensais em algumas estações européias. Podemos usar o modelo para analisar a relação entre as séries $X(t)$ e $Y(t)$ e testar se existe algum efeito de manchas solares sobre as temperaturas nas estações européias. Este exemplo é citado por Brillinger(1969). Neste caso, $\mathrm{N}=$ número de estações utilizadas e $P=1$.

Em geral, nosso objetivo é verificar se existe efeito de algum subconjunto de $X(t)$ sobre $Y(t)$, ou seja, particionamos $X(t)$ em $P_{1}$ e $P_{2}$ componentes, $X(t)=\left(X_{1}(t), X_{2}(t)\right)^{\prime}$ e $\theta(u)=\left(\theta_{1}(u), \theta_{2}(u)\right)^{\prime}$, transformando o modelo (4.93) em

$$
Y(t)=\mu+\sum_{u} \theta_{1}(u) X_{1}(t-u)+\sum_{u} \theta_{2}(u) X_{2}(t-u)+\epsilon(t)
$$

e testamos a hipótese

$$
H_{18}: \theta_{2}(\cdot)=0
$$

Um procedimento para fazer o teste (4.96) é comparar o modelo completo (4.95) com o modelo sob a hipótese $H_{18}$, isto é

$$
Y(t)=\mu+\sum_{u} \theta_{1}(u) X_{1}(t-u)+\epsilon(t) .
$$

Para $k=1, \cdots, K, k \neq 0, \pi$ e $\frac{2 \pi k}{T} \simeq \lambda$, o modelo (4.93) pode ser representado em termos da transformada discreta de Fourier

$$
Y^{\wedge}(k)=\Theta(\lambda) X^{\wedge}(k)+\xi_{k}+O_{a . s .}(1)
$$

onde

$$
\begin{aligned}
& X^{\wedge}(k)=\left(X_{1}^{\wedge}(k), X_{2}^{\wedge}(k)\right)^{\prime} ; \\
& \Theta(\lambda)=\left(\Theta_{1}(\lambda), \Theta_{2}(\lambda)\right)^{\prime}=\sum_{u} \exp \{-i \lambda u\} \theta(u) ;
\end{aligned}
$$

$\xi_{k}$ : variávies $N^{c}\left(0, f_{\epsilon \epsilon}(\lambda)\right)$ independentes. 
Utilizando (4.98), o teste para (4.96) pode ser realizado através da hipótese

$$
H_{19}: \Theta_{2}(\cdot)=0 \text {. }
$$

Supondo que $f_{x x}^{(T)}(\lambda)$ é não singular, as estimativas de $\Theta(\lambda)$ e $f_{\epsilon \epsilon}(\lambda)$ podem ser obtidas através de

$$
\Theta^{\wedge}(\lambda)=Y^{\wedge}(k){\overline{X^{\wedge}(k)}}^{\prime}\left(X^{\wedge}(k){\overline{X^{\wedge}(k)}}^{\prime}\right)^{-1}=f_{y x}^{(T)}(\lambda)\left(f_{x x}^{(T)}(\lambda)\right)^{-1}
$$

$\mathrm{e}$

$$
f_{\epsilon \epsilon}^{(T)}(\lambda)=\frac{K}{K-P}\left\{f_{y y}^{(T)}(\lambda)-f_{y x}^{(T)}(\lambda)\left(f_{x x}^{(T)}(\lambda)\right)^{-1} f_{x y}^{(T)}(\lambda)\right\}
$$

Para podemos fazer o teste (4.99) precisamos de alguns resultados dados a seguir (Brillinger, 1969 e Mardia et al, 1979).

Teorema 4.1: Suponha que $Y(t), t=0, \pm 1, \cdots$, satisfaz o modelo (4.93) e que $f_{x x}^{(T)}\left(\lambda_{j}\right), j=1, \cdots, J$, são matrizes não singulares para $T$ suficientemente grande, então

$$
V e c\left[\left\{\Theta^{\wedge}\left(\lambda_{j}\right)-\Theta\left(\lambda_{j}\right)\right\}\left\{f_{x x}^{(T)}\left(\lambda_{j}\right)\right\}^{\frac{1}{2}}\right] \stackrel{\mathcal{D}}{\rightarrow} N_{N P}^{c}\left(0, K^{-1} f_{\epsilon \epsilon}(\lambda) \otimes I\right\}
$$

$\mathrm{e}$

$$
f_{\epsilon \epsilon}^{(T)}\left(\lambda_{j}\right) \stackrel{\mathcal{D}}{\rightarrow}(K-P)^{-1} W_{N}^{c}\left(K-P, f_{\epsilon \epsilon}\left(\lambda_{j}\right)\right) .
$$

Além disso, se $\lambda_{j} \pm \lambda_{k} \not \equiv 0(\bmod 2 \pi), j=1, \cdots, J, f_{\epsilon \epsilon}^{(T)}\left(\lambda_{j}\right)$ e $\left\{\Theta^{(T)}\left(\lambda_{j}\right)-\right.$ $\left.\Theta\left(\lambda_{j}\right)\right\}\left\{f_{x x}^{(T)}\left(\lambda_{j}\right)\right\}^{\frac{1}{2}}$ são assintoticamente independentes.

Corolário 4.1: Sob as mesmas condições do Teorema 4.1,

$$
\left\{\Theta^{\wedge}(\lambda)-\Theta(\lambda)\right\}\left\{f_{x x}^{(T)}(\lambda)\right\}\left\{\Theta^{\wedge}(\lambda)-\Theta(\lambda)\right\}^{\prime} \stackrel{\mathcal{D}}{\rightarrow} K^{\prime-1} W_{N}^{c}\left(P, f_{\epsilon \epsilon}(\lambda)\right)
$$

quando $T \rightarrow \infty$ e é independente de $f_{\epsilon \epsilon}(\lambda)$.

Definição 4.1: Quando $A \sim W_{N}(Q, \Sigma)$ e $B \sim W_{N}(P, \Sigma)$ são independentes, $Q \geq N$, dizemos que

$$
\Lambda_{(N, Q, P)}=\frac{|A|}{|A+B|}=\left|I+A^{-1} B\right|^{-1}
$$


tem uma distribuição lambda de Wilks (Wilk's lambda) com parâmetros $N$, $Q$ e $P$.

Definição 4.2: Quando $A \sim W_{N}^{c}(Q, \Sigma)$ e $B \sim W_{N}^{c}(P, \Sigma)$ são independentes, $Q \geq N$, dizemos que

$$
\Lambda_{(N, 2 Q, 2 P)}=\frac{|A|}{|A+B|}=\left|I+A^{-1} B\right|^{-1}
$$

tem uma distribuição lambda de Wilks com parâmetros $N, 2 Q$ e $2 P$.

Para fazer a análise consideraremos as seguintes somas de quadrados:

$$
\begin{gathered}
S Q H_{c}(\lambda)=Y^{\wedge}(k){\overline{X^{\wedge}(k)}}^{\prime}\left(X^{\wedge}(k){\overline{X^{\wedge}(k)}}^{\prime}\right)^{-1} X^{\wedge}{\overline{Y^{\wedge}(k)}}^{\prime}, \\
S Q H_{19}(\lambda)=Y^{\wedge}(k){\overline{X_{1}^{\wedge}(k)}}^{\prime}\left(X_{1}^{\wedge}(k){\overline{X_{1}^{\wedge}(k)}}^{\prime}\right)^{-1} X_{1}^{\wedge}{\overline{Y^{\wedge}(k)}}^{\prime}, \\
S Q H_{\theta_{2}}(\lambda)=S Q H_{c}(\lambda)-S Q H_{19}(\lambda) \\
S Q R_{c}(\lambda)=Y^{\wedge}(k){\overline{Y^{\wedge}(k)}}^{\prime}-Y^{\wedge}(k){\overline{X^{\wedge}(k)}}^{\prime}\left(X^{\wedge}(k){\overline{X^{\wedge}(k)}}^{\prime}\right)^{-1} X^{\wedge} \overline{Y^{\wedge}(k)}
\end{gathered}
$$

Segundo o Teorema 4.1 e o Corolário 4.1 , sob $H_{19}$,

$$
S Q R_{c}(\lambda) \stackrel{\mathcal{D}}{\rightarrow} K^{-1} W_{N}^{c}\left(K-P, f_{\epsilon \epsilon}(\lambda)\right)
$$

$\mathrm{e}$

$$
S Q H_{\theta_{2}}(\lambda) \stackrel{\mathcal{D}}{\rightarrow} K^{-1} W_{N}^{c}\left(P_{2}, f_{\epsilon \epsilon}(\lambda)\right)
$$

independentes. Segundo a Definição 4.2, o teste para (4.99) pode ser realizado utilizando a estatística

$$
\Lambda_{\left(N, 2(K-P), 2 P_{2}\right)}=\frac{S Q R_{c}(\lambda)}{S Q R_{c}(\lambda)+S Q H_{\theta_{2}}(\lambda)}
$$

que, sob $H_{19}$, tem uma distribuição $\Lambda_{\left(N, 2(K-P), 2 P_{2}\right)}$.

Os resultados obtidos estão resumidos nas Tabclas 4.10 e 4.11. Para mais detalhes, ver Roy et al(1971) e Krishnaiah(1969).

Para utilizarmos a estatística $\Lambda$, precisamos dos seguintes resultados. 
Teorema 4.2: Temos que

$$
\Lambda_{(N, Q, P)} \sim \prod_{i=1}^{P} \mu_{i}
$$

onde $\mu_{1}, \cdots, \mu_{P}$ são $P$ variáveis independentes, e

$$
\mu_{i} \sim \beta\left(\frac{1}{2}(Q+i-N), \frac{1}{2} N\right), \quad i=1, \cdots, P .
$$

Segundo as relações entre as variáveis $\beta$ e $F$, as estatísticas $\Lambda_{(1, Q, P)}$ e $\Lambda_{(2, Q, P)}$ podem ser expressadas como

$$
\frac{1-\Lambda_{(1, Q, P)}}{\Lambda_{(1, Q, P)}} \sim \frac{P}{Q} F(P, Q)
$$

e

$$
\frac{1-\sqrt{\Lambda_{(2, Q, P)}}}{\sqrt{\Lambda_{(2, Q, P)}}} \sim \frac{P}{Q-1} F(2 P, 2(Q-1)) .
$$

Para outros valores de $N$, temos

$$
-\left\{Q-\frac{1}{2}(N-P+1)\right\} \log \Lambda_{(N, Q, P)} \stackrel{Q \rightarrow \infty}{\longrightarrow} \chi_{P N}^{2} .
$$

Ver Mardia et al(1979) e Anderson(1958).

Para $N=1$, a estatística (4.109) pode ser transformada de acordo com

$$
\begin{aligned}
\frac{1-\Lambda_{\left(1,2(K-P), 2 P_{2}\right)}}{\Lambda_{\left(1,2(K-P), 2 P_{2}\right)}} & =\frac{1-S Q R_{c}(\lambda) /\left(S Q R_{c}(\lambda)+S Q H_{\theta_{2}}(\lambda)\right)}{S Q R_{c}(\lambda) /\left(S Q R_{c}(\lambda)+S Q H_{\theta_{2}}(\lambda)\right)} \\
& =\frac{S Q H_{\theta_{2}}(\lambda)}{S Q R_{c}(\lambda)} \\
& \sim \frac{2 P_{2}}{2(K-P)} F\left(2 P_{2}, 2(K-P)\right),
\end{aligned}
$$

ou seja,

$$
\frac{S Q H_{\theta_{2}}(\lambda) / 2 P_{2}}{S Q R_{c}(\lambda) / 2(K-P)} \sim F\left(2 P_{2}, 2(K-P)\right)
$$


que é equivalente à estatística (4.89). Isto é, o modelo (4.83) é um caso particular do (4.98) quando $N=1$.

Para $N=2$, a estatística (4.109) pode ser transformada como

$$
\frac{\left(1-\sqrt{\Lambda_{\left(2,2(K-P), 2 P_{2}\right)}}\right) / 2 P_{2}}{\sqrt{\Lambda_{\left(2,2(K-P), 2 P_{2}\right)}} /(2(K-P)-1)} \sim F\left(4 P_{2}, 2(2 K-2 P-1)\right) .
$$

Tabela 4.10: MANOVAF para o teste (4.99) na frequência $\lambda$

\begin{tabular}{ll}
\hline \hline fonte & \multicolumn{1}{c}{$\begin{array}{c}\text { soma de quadrados } \\
(S Q)\end{array}$} \\
\hline Modelo reduzido & $Y^{\wedge}(k){\overline{X_{1}^{\wedge}(k)}}^{\prime}\left(X_{1}^{\wedge}(k){\overline{X_{1}^{\wedge}(k)}}^{\prime}\right)^{-1} X_{1}^{\wedge}(k){\overline{Y^{\wedge}(k)}}^{\prime}(\star)$ \\
Modelo só com $\theta_{2}(t)$ & $(\star \star)-(\star)$ \\
\hline Modelo completo & $Y^{\wedge}(k){\overline{X^{\wedge}(k)}}^{\prime}\left(X^{\wedge}(k){\overline{X^{\wedge}(k)}}^{\prime}\right)^{-1} X^{\wedge}(k){\overline{Y^{\wedge}(k)}}^{\prime}(\star \star)$ \\
Resíduo & $(\star \star \star)-(\star \star)$ \\
\hline Total & $Y^{\wedge}(k){\overline{Y^{\wedge}(k)}}^{\prime}(\star \star \star)$ \\
\hline \hline
\end{tabular}

\begin{tabular}{lccc}
\hline \hline fonte & grau de liberdade & quadrado médio=2SQ/gl & estatística \\
& $(g l)$ & $(Q M)$ & $\Lambda$ \\
\hline Modelo reduzido & $2 P_{1}$ & $\frac{K f_{y x}^{(T)}(\lambda)\left(f_{x x 1}^{(T)}(\lambda)\right)^{-1} f_{x y y}^{(T)}(\lambda)}{P_{1}}$ & \\
Modelo só com $\theta_{2}(t)$ & $2 P_{2}$ & & $\frac{(\star \star \star)-(\star \star)}{(\star \star \star)-(\star)}$ \\
\hline Modelo completo & $2 P$ & $\frac{K f_{y x}^{(T)}(\lambda)\left(f_{x x}^{(T)}(\lambda)\right)^{-1} f_{x y}^{(T)}(\lambda)}{P}$ & \\
Resíduo & $2(K-P)$ & $\frac{K\left[f_{y y}^{(T)}(\lambda)-f_{y x}^{(T)}(\lambda)\left(f_{x x}^{(T)}(\lambda)\right)^{-1} f_{x y}^{(T)}(\lambda)\right]}{K-P}$ & \\
\hline Total & $2 K$ & & \\
\hline \hline
\end{tabular}


Tabela 4.11: Quadrado médio esperado na frequência $\lambda$ assumindo que $\Theta_{2}(\lambda)=0$

\begin{tabular}{ll}
\hline \hline fonte & $\begin{array}{c}\text { quadrado médio esperado } \\
(E Q M)\end{array}$ \\
\hline Modelo reduzido & $f_{\epsilon \epsilon}(\lambda)+\frac{K \Theta_{1}(\lambda) f_{x_{1} x_{1}}^{(T)}(\lambda) \overline{\Theta_{1}(\lambda)}}{P_{1}}$ \\
Modelo só com $\theta_{2}(t)$ & $f_{\epsilon \epsilon}(\lambda)$ \\
\hline Modelo completo & $f_{\epsilon \epsilon}(\lambda)+\frac{K \Theta(\lambda) f_{x x}^{(T)}(\lambda) \overline{\Theta_{(\lambda)}}}{P}$ \\
Resíduo & $f_{\epsilon \epsilon}(\lambda)$ \\
\hline \hline
\end{tabular}




\section{Capítulo 5}

\section{Análise de Variância Usando a Transformada de Walsh-Fourier}

\subsection{Introdução}

$\mathrm{Na}$ análise de séries temporais, o método de Fourier é usado para descobrir e analisar a regularidade ou periodicidade nos dados. Esta técnica formaliza o conceito de dependência ou correlação entre os pontos adjacentes numa série temporal. Por exemplo, a Figura 5.1 mostra a média mensal de 24 leituras diárias da temperatura de São Paulo, no período de julho de 1949 até junho de 1957, obtidas pelo IAG-USP. A figura mostra claramente um comportamento senoidal com um período de 12 meses. Este é um exemplo simples, onde o ciclo ou período nos dados é observado através de uma análise visual. Nos problemas mais complicados, precisamos de um método mais preciso de identificação, que possibilite uma análise estatística dos componentes harmônicos das variações apresentadas nos dados. A análise de Fourier é usada neste caso.

Mas nem todos os comportamentos periódicos são senoidais, por exemplo, a Figura 5.2 mostra um eletroencefalograma(EEG) durante o estado de sono de uma criança, cuja mãe não consumiu bebidas alcoólicas durante sua gravidez. Podemos notar que os dados não são senoidais, eles apresentam um comportamento na forma de "ondas quadradas". Neste caso, é mais conveniente descrevê-los em termos das chamadas funções de Walsh, que são apresentadas na Figura 5.3 e tal procedimento é denominado uma análise de Walsh-Fourier. 


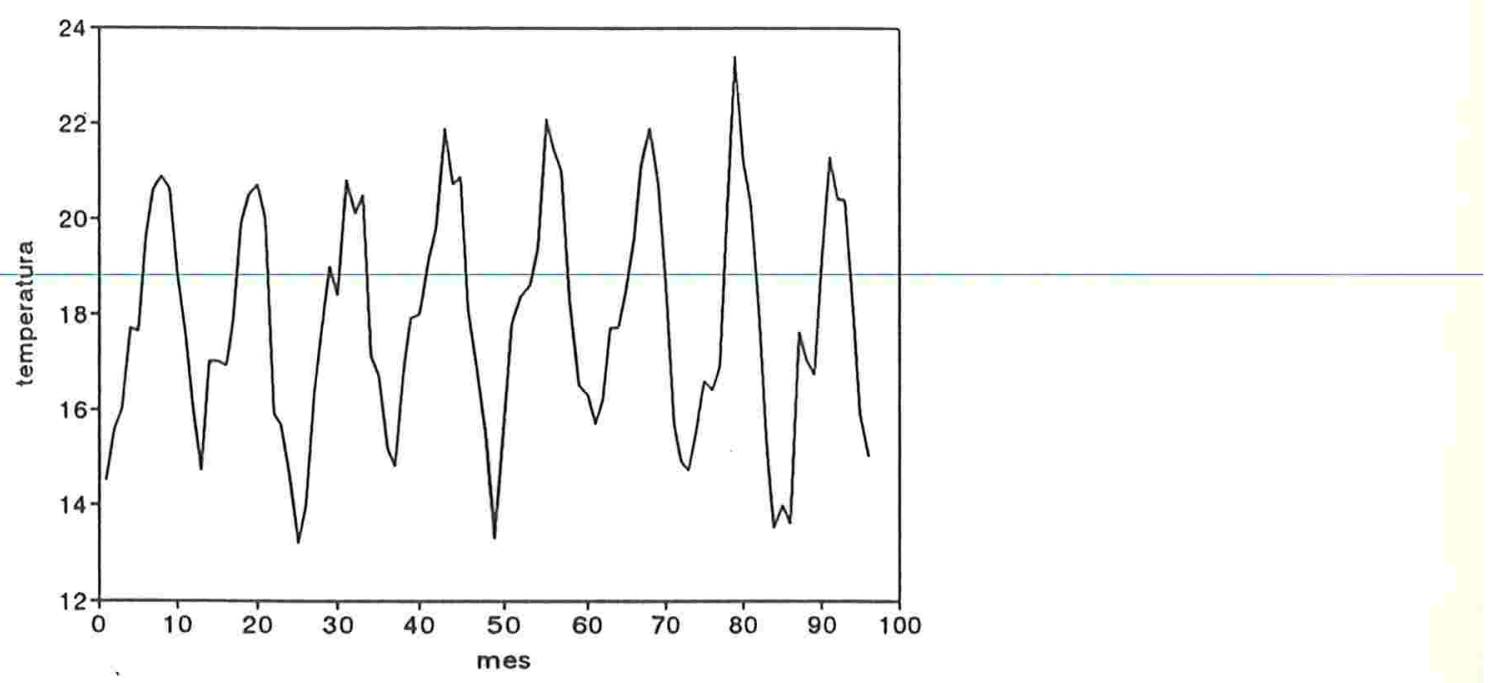

Figura 5.1: Média mensal de 24 leituras diárias da temperatura de São Paulo

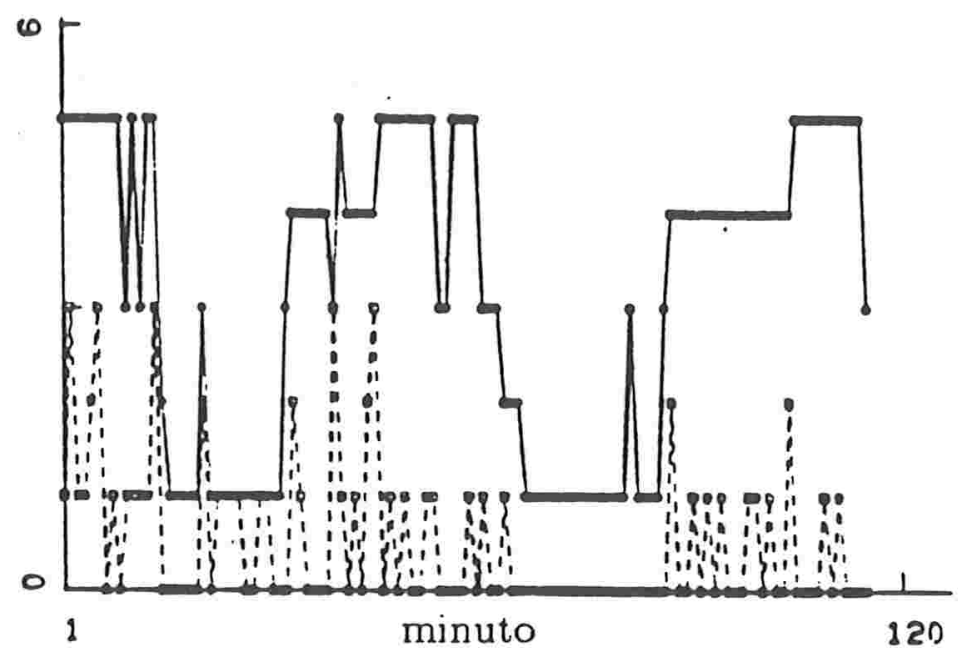

Figura 5.2: Eletroencefalograma durante o estado de sono de uma criança cuja mãe não consumiu bebidas alcoólicas durante sua gravidez 
A análise de Walsh-Fourier não é uma substituição da análise de Fourier, nem é uma cópia dela. Cada uma das técnicas tem suas próprias vantagens e desvantagens; além disso, nem sempre é necessário escolher entre um dos dois métodos, pois ambos podem ser aplicados em algumas situações. Beauchamp $(1975,1984)$ concluiu que quando o sinal da série temporal tem aspecto senoidal ou apresenta alguma forma de periodicidade que possa ser encarada como uma soma de ondas senoidais, a análise de Fourier é relevante e quando o sinal contém descontinuidades e um número limitado de níveis ("ondas quadradas"), a análise de Walsh é apropriada.

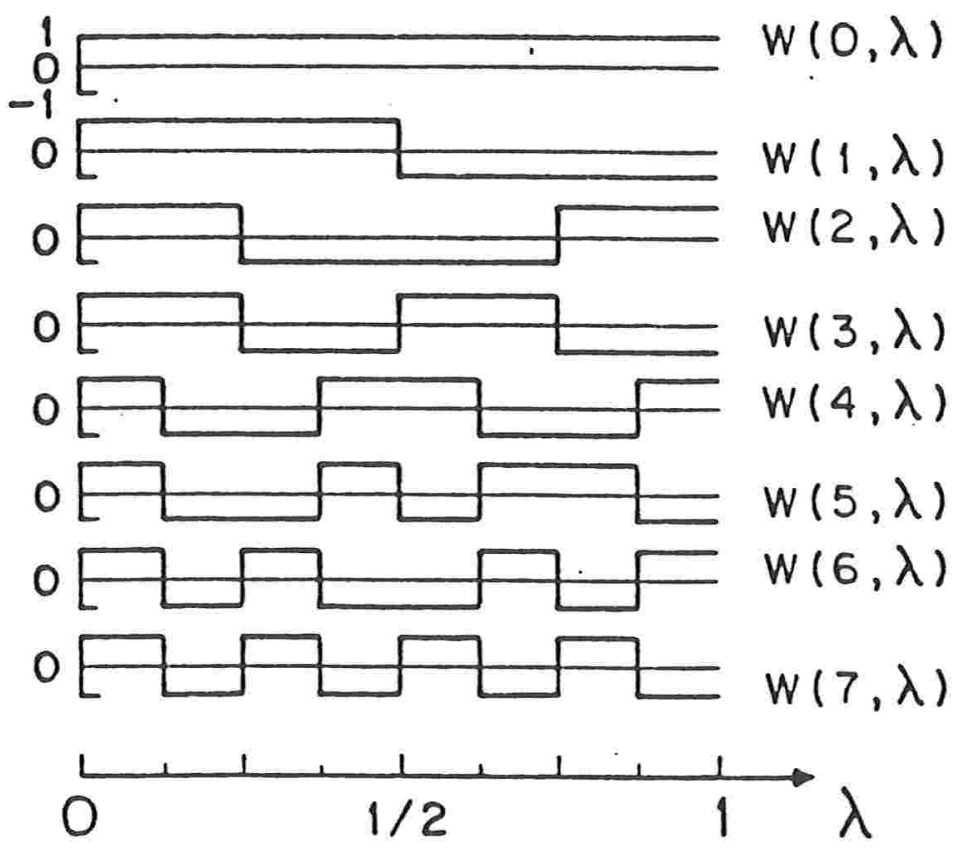

Figura 5.3: As Funções de Walsh

\subsection{Análise no domínio de sequências}

As funções de Walsh, que são definidas através das funções de Rademacher (ver Capítulo 3), formam uma sequência ortonormal completa no intervalo $[0,1)$ e assumem somente dois valores, +1 e -1 . Elas são ordenadas pelo número de "zero-crossings" ( ou sinais trocados), denominado sequência. 
Seja $W(n, \lambda), n=0,1,2, \cdots, 0 \leq \lambda<1$, a n-ésima sequência ordenada da função de Walsh, então $W(n, \cdot)$ produz n "zero-crossings" em $[0,1)$. Os oitos primeiros valores discretos, ordenados segundo a sequência da função de Walsh $W\left(n, \frac{m}{T}\right), m, n=0,1, \cdots, 7$ correspondendo ao tamanho de amostra $T=2^{3}$, são mostrados na Figura 5.4 numa matriz simétrica, de dimensão $8 \times 8$, chamada matriz ordenada de Walsh-Hadamard, $H_{w}(3)$. Na análise estatística de séries temporais, serão utilizadas as funções discretas de Walsh.

$$
H_{w}(3)=\left[\begin{array}{rrrrrrrr}
1 & 1 & 1 & 1 & 1 & 1 & 1 & 1 \\
1 & 1 & 1 & 1 & -1 & -1 & -1 & -1 \\
1 & 1 & -1 & -1 & -1 & -1 & 1 & 1 \\
1 & 1 & -1 & -1 & 1 & 1 & -1 & -1 \\
1 & -1 & -1 & 1 & 1 & -1 & -1 & 1 \\
1 & -1 & -1 & 1 & -1 & 1 & 1 & -1 \\
1 & -1 & 1 & -1 & -1 & 1 & -1 & 1 \\
1 & -1 & 1 & -1 & 1 & -1 & 1 & -1
\end{array}\right]
$$

Figura 5.4: Matriz ordenada de Walsh-Hadamard

A relação entre as funções de Walsh $\Psi(n, \lambda)$ e as funções ordenadas de Walsh $W(n, \lambda)$ é

$$
W(n, \lambda)= \begin{cases}\Psi\left(\frac{n}{2} \oplus n, \lambda\right) & n=0,2,4, \cdots \\ \Psi\left(\frac{n-1}{2} \oplus n, \lambda\right) & n=1,3,5, \cdots\end{cases}
$$

Propriedades DE $W(n, \lambda)$ :

(i) $W(i, \lambda) W(k, \lambda)=W(i \oplus k, \lambda)$.

(ii) $W(i, \lambda) W(i, \lambda)=W(0, \lambda)$.

(iii) $W(i, \lambda) W(0, \lambda)=W(i, \lambda)$.

(iv) $[W(i, \lambda) W(j, \lambda)] W(k, \lambda)=W(i, \lambda)[W(j, \lambda) W(k, \lambda)]$.

Atualmente, existem dois métodos de desenvolvimento de análise espectral de Walsh na literatura. O primeiro método é chamado análise espectral 
de Walsh e é aplicado através do conceito de série estacionária diádica. $\mathrm{O}$ outro método é chamado análise espectral de Walsh-Fourier e é baseado em séries estacionárias.

As idéias básicas da análise de Fourier são utilizadas na análise de WalshFourier. As funções de Walsh são similares ao sistema de senos e cossenos usado na análise de Fourier. Mas, ao contrário dos componentes senoidais, as funções de Walsh são na forma de "ondas quadradas" que assumem somente dois valores, +1 e -1 . As senoidais na análise de Fourier, $\cos (2 \pi n \lambda)$ e $\operatorname{sen}(2 \pi n \lambda), n=1,2, \cdots$, são diferenciadas pela sua frequência de oscilação $\mathrm{n}$, em termos do número de ciclos completos que ela produz no intervalo $0 \leq \lambda<1$. Por exemplo, quando $\mathrm{n}=3, \cos (2 \pi n \lambda)$ e $\operatorname{sen}(2 \pi n \lambda)$ completam três ciclos no intervalo $0 \leq \lambda<1$. As funções de Walsh (Figura 5.5), denotadas por $W(n, \lambda)(n=0,1,2, \cdots, 0 \leq \lambda<1)$, são diferenciadas pelo número de vezes $\mathrm{n}$ que elas trocam de valor no intervalo unitário. Por exemplo, $W(3, \lambda)$ troca três vezes de valor no intervalo $0 \leq \lambda<1$.

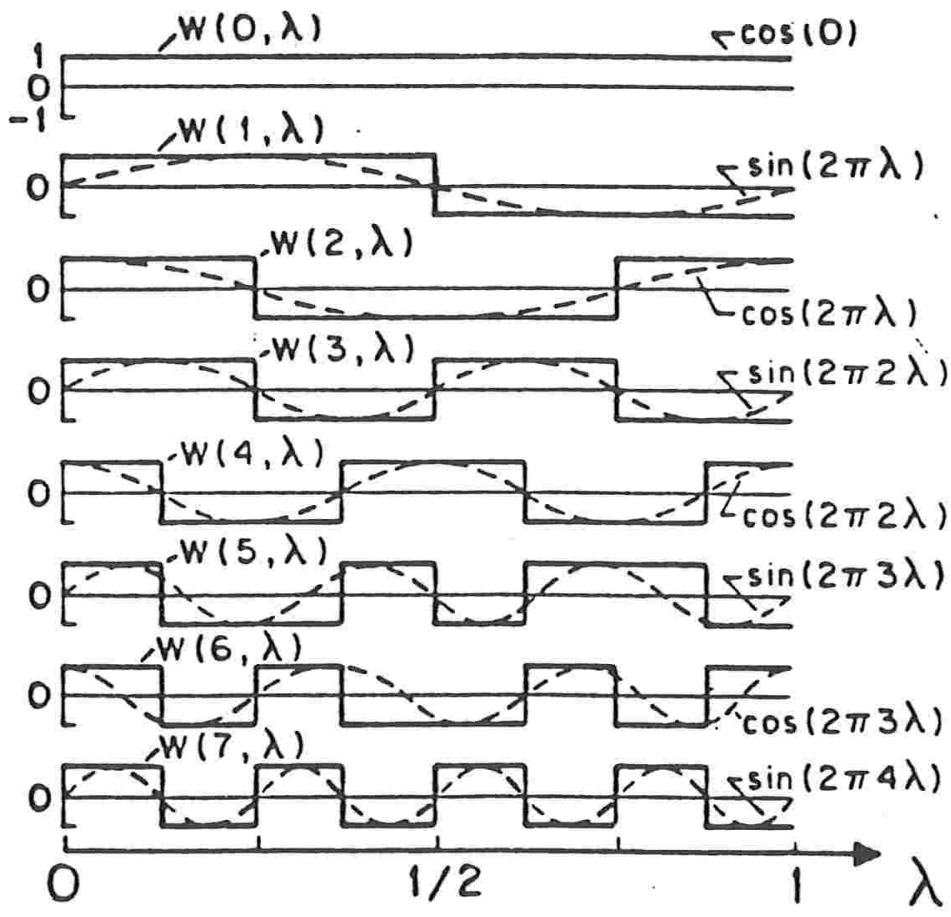

Figura 5.5: Walsh e Fourier (Harmônicos) 
A Figura 5.5 mostra a superposição das funções de Fourier e Walsh. Podemos notar que a frequência $\mathrm{n}$ em $\cos (2 \pi n \lambda)$ e $\operatorname{sen}(2 \pi n \lambda)$ também pode ser interpretada como a metade do número de "troca de sinal" por unidade de tempo. Por exemplo, na Figura 5.5, quando $\mathrm{n}=2, \cos (2 \pi n \lambda)$ e $\operatorname{sen}(2 \pi n \lambda)$ cruzam o eixo (ou mudam de sinal) quatro vezes. Para fazer a analogia entre frequência e número de "zero-crossings" ou sinais trocados em funções periódicas, define-se a "sequência de Harmuth" como a metade do número de "zero-crossings" ou sinais trocados, que uma função produz por unidade de tempo. Esse conceito pode ser aplicado tanto para funções aperiódicas como para funções periódicas. Assim, a definição da "sequência de Harmuth" coincide com a definição de frequência quando aplicado em funções senoidais. Harmuth(1969b) definiu as funções de Walsh-cosseno(Cal) e Walsh-seno(Sal) para promover analogia entre a análise de Walsh e a de Fourier.

As funções, Cal e Sal são definidas por

(i) $\operatorname{Cal}(i, \lambda)=W(2 i, \lambda), i=1,2,3, \cdots$;

(ii) $\operatorname{Cal}(0, \lambda)=1$;

(iii) $\operatorname{Sal}(i, \lambda)=W(2 i-1, \lambda), i=1,2,3, \cdots$, para $-\infty<\lambda<\infty$.

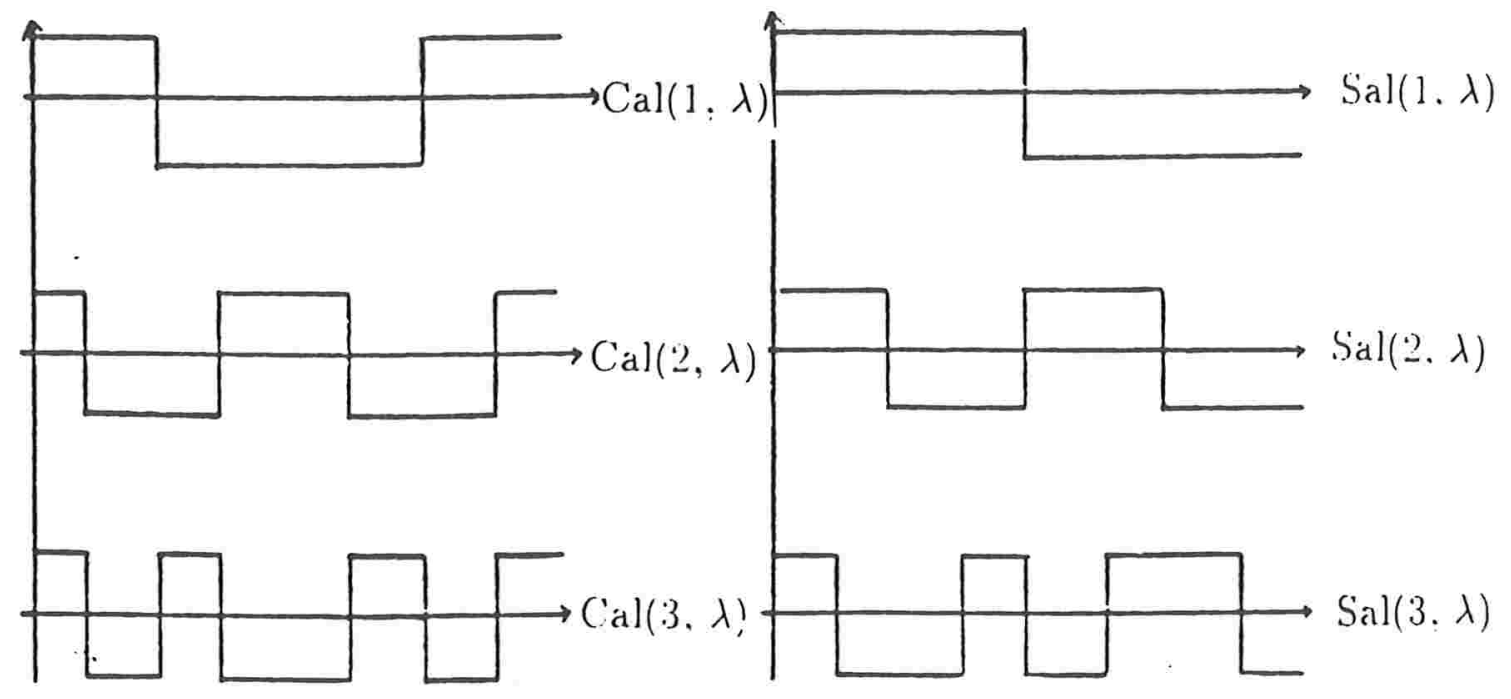

Figura 5.6: As funções Cal e Sal para $i=1,2,3$ 


\subsection{Comparação entre as análises de Fourier e Walsh-Fourier}

Utilizamos a "sequência de Harmuth", denotada "H-sequency", para fazer as comparações entre a análise de Fourier e a de Walsh-Fourier.

A função discreta de Walsh $W(n, \lambda)$ produz $n$ "zero crossings" por unidade de tempo, então seu valor de sequência é $n$. A "H-sequency" desta função é $\frac{n}{2}$ se $n$ é par, e $\frac{n+1}{2}$ se $n$ é impar. Portanto, as funções de Walsh $W(2 n, \lambda)$ e $W(2 n-1, \lambda)$ têm mesma "H-sequency", isto é, $W(2 n, \lambda)$ e $W(2 n-1, \lambda)$, cada uma delas tem uma "H-sequency" igual a $n$. Isto também vale para as funções senoidais, $\cos (2 \pi n \lambda)$ e $\operatorname{sen}(2 \pi n \lambda)$ têm frequência igual a $n$. Podemos notar que a "H-sequency" e a frequência coincidem em senoidais e, portanto, o periodograma de Walsh-Fourier, definido em (3.39), pode ser modificado da seguinte maneira

$$
I_{x x}^{(T, H)}\left(\lambda_{j}\right)=I_{x x}^{(T, s)}\left(\lambda_{2 j-1}\right)+I_{x x}^{(T, s)}\left(\lambda_{2 j}\right)
$$

para $j=1,2, \cdots, \frac{T-2}{2}$, onde $\lambda_{j}=\frac{j}{T}$ representa "H-sequency" e,

$$
\begin{aligned}
I_{x x}^{(T, s)}\left(\lambda_{2 j-1}\right) & =T^{-1}\left[\sum_{t=0}^{T-1} X(t) W\left(t, \lambda_{2 j-1}\right)\right]^{2} \\
I_{x x}^{(T, s)}\left(\lambda_{2 j}\right) & =T^{-1}\left[\sum_{t=0}^{T-1} X(t) W\left(t, \lambda_{2 j}\right)\right]^{2} .
\end{aligned}
$$

O periodograma de Walsh-Fourier, dado por (5.1), é correspondente ao periodograma de Fourier dado por (2.23).

DemonstraÇ̃̃o: Como $\lambda_{2 j-1}=\frac{2 j-1}{T}$, então $W\left(n, \lambda_{2 j-1}\right)=W\left(n, \frac{2 j-1}{T}\right)$. Utilizando (3.15), podemos escrever

$$
W\left(n, \frac{2 j-1}{T}\right)=W\left(2 j-1, \frac{n}{T}\right)=\operatorname{Sal}\left(j, \frac{n}{T}\right),
$$


que é equivalente ao $\operatorname{sen}\left(2 \pi j \frac{n}{T}\right)$ (ver Figura 5.5). Assim,

$$
\operatorname{Sal}\left(j, \frac{n}{T}\right) \sim \operatorname{sen}\left(2 \pi j \frac{n}{T}\right)=\operatorname{sen}\left(2 \pi n \frac{j}{T}\right)=\operatorname{sen}\left(2 \pi n \lambda_{j}\right),
$$

$\mathrm{e}$

$$
W\left(n, \lambda_{2 j-1}\right) \sim \operatorname{sen}\left(2 \pi n \lambda_{j}\right) .
$$

Analogamente, temos

$$
W\left(n, \lambda_{2 j}\right)=W\left(n, \frac{2 j}{T}\right)=W\left(2 j, \frac{n}{T}\right)=\operatorname{Cal}\left(j, \frac{n}{T}\right),
$$

que é equivalente ao $\cos \left(2 \pi j \frac{n}{T}\right)$ (ver Figura 5.5). Portanto,

$$
\operatorname{Cal}\left(j, \frac{n}{T}\right) \sim \cos \left(2 \pi j \frac{n}{T}\right)=\cos \left(2 \pi n \frac{j}{T}\right)=\cos \left(2 \pi n \lambda_{j}\right),
$$

e podemos dizer que

$$
W\left(n, \lambda_{2 j}\right) \sim \cos \left(2 \pi n \lambda_{j}\right)
$$

Agora,

$$
\begin{aligned}
I_{x x}^{(T, H)}\left(\lambda_{j}\right) & =I_{x x}^{(T, s)}\left(\lambda_{2 j-1}\right)+I_{x x}^{(T, s)}\left(\lambda_{2 j}\right) \\
& =T^{-1}\left[\sum_{t=0}^{T-1} X(t) W\left(t, \lambda_{2 j-1}\right)\right]^{2}+T^{-1}\left[\sum_{t=0}^{T-1} X(t) W\left(t, \lambda_{2 j}\right)\right]^{2} \\
& \sim T^{-1}\left[\sum_{t=0}^{T-1} X(t) \operatorname{sen}\left(2 \pi t \lambda_{j}\right)\right]^{2}+T^{-1}\left[\sum_{t=0}^{T-1} X(t) \cos (2 \pi t \lambda j)\right]^{2} \\
& =2 \pi I_{x x}^{(T)}\left(\lambda_{j}\right)
\end{aligned}
$$

que é proporcional ao periodograma de Fourier (2.23).

O EEG durante o estado de sono, mostrado na Figura 5.2, é analisado utilizando a transformada rápida de Fourier (FFT) e de Walsh-Fourier (FWT). (ver Ahmed e Rao, 1975). Tomamos $T=128=2^{7}$ para poder utilizar FFT e FWT. O periodograma de Fourier e de Walsh-Fourier são apresentados na Figura 5.7. Como $\sum_{j} I_{x x}^{(T, H)}\left(\lambda_{j}\right) \sim 2 \sum_{j} I_{x x}^{(T)}\left(\lambda_{j}\right)$, excluindo os pontos $\lambda_{j}=0$ e $\lambda_{j}=1 / 2, I_{x x}^{(T, H)}\left(\lambda_{j}\right) / 2$ e $I_{x x}^{(T)}\left(\lambda_{j}\right)$ são superpostos para facilitar a comparação. Para a melhor comparação visual, a Figura 5.7 apresenta 
$10 \log \left\{I_{x x}^{(T, H)}\left(\lambda_{j}\right) / 2+1\right\}$ e $10 \log \left\{I_{x x}^{(T)}\left(\lambda_{j}\right)+1\right\}, \operatorname{com} \lambda_{j}=\frac{j}{T}$, para $j=1, \cdots, 7$, e $I_{x x}^{(T, H)}\left(\lambda_{j}\right) / 2$ e $I_{x x}^{(T)}\left(\lambda_{j}\right)$ para $j=8, \cdots, 63$.

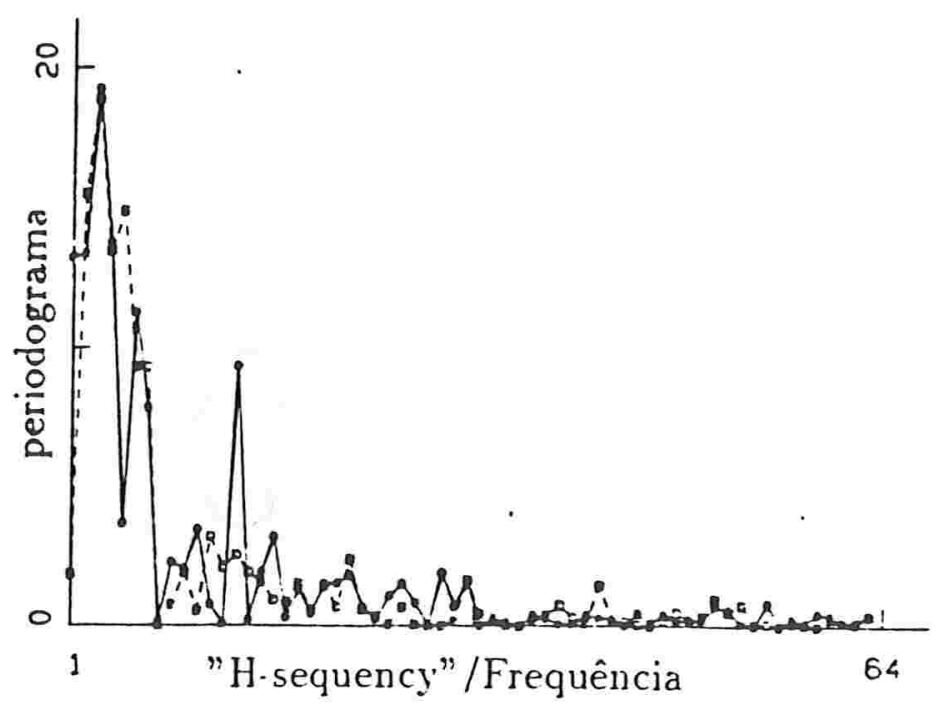

Figura 5.7: Comparação do periodograma de Walsh (círculos) e de Fourier (quadrados) para os dados mostrados na Figura 5.2

Podemos notar que os dois periodogramas são muito similares, e ambos apresentam um forte pico em torno de $\lambda=3 / 128$. No domínio de frequência, $\lambda$ é medido em ciclos por minuto, isto é, o componente harmônico principal nos dados é aproximadamente 43 minutos (128/3 minutos) por ciclo. No domínio de "H-sequency", Harmuth(1977) interpreta a quantidade 43 minutos como sendo o período médio das oscilações dominantes, isto é, o intervalo médio dos "zero crossings" multiplicado por 2.

A diferença mais notável entre os dois periodogramas é o pico que aparece no periodograma de Walsh-Fourier em torno de $\lambda=14 / 128$ e não aparece no periodograma de Fourier. Esta "H-sequency" corresponde a um período de aproximadamente 9 minutos. Notamos, na Figura 5.2, que o intervalo médio que uma criança fica em qualquer estado é aproximadamente 4,52 minutos. Portanto, o intervalo médio das mudanças de estado multiplicado por 2 é 9 minutos. Este componente é perdido na análise de Fourier. 


\subsection{Análise de variância usando Walsh-Fourier}

Consideremos agora o problema de detectar se existe ou não um sinal comum em $N$ réplicas de uma série temporal discreta $\left\{X_{n}(t), n=1,2, \cdots, N, t=\right.$ $0,1, \cdots, T-1\}$.

Por exemplo, suponha que queremos analisar a situação de similaridade de $N$ filas independentes num banco durante um certo intervalo. Seja $X_{n}(t)$, $n=1,2, \cdots, N, t=0,1, \cdots, T-1$, o número de indivíduos na fila $n$ no instante $t$. Cada $X_{n}(t)$ pode ser decomposto na soma de três componentes: um valor médio $\Theta_{n}$, que depende só da fila, um sinal comum estacionário $\Omega(t)$ para todas as filas, no instante $t$, representando a variação do número de indivíduos numa fila, e um erro estacionário $\epsilon_{n}(t)$, isto é,

$$
X_{n}(t)=\Theta_{n}+\Omega(t)+\epsilon_{n}(t)
$$

onde $\Omega(t)$ tem média zero e espectro de Walsh-Fourier $S_{\Omega}^{(w)}(\lambda), \epsilon_{n}(t)$ tem média zero e espectro de Walsh-Fourier $S_{\epsilon}^{(w)}(\lambda)$. Além disso, esses dois componentes são não correlacionados.

Testar a existência de similaridade entre as filas num banco, é equivalente testar a hipótese nula $H_{0}: S_{\Omega}^{(w)}(\lambda)=0,(0<\lambda<1)$.

De um modo geral, suporemos que o sistema é da forma $X_{n}(t), n=$ $1, \cdots, N, t=0,1, \cdots, T-1, T=2^{p}, p>0$ inteiro, e pode ser modelado por (5.6), onde:

$\Theta_{n}:$ constantes;

$\Omega(t)$ : uma série temporal estacionária com média zero, funções de autocovariância $R_{\Omega \Omega}(\tau)$ e covariância lógica $\Gamma_{\Omega \Omega}(j)$ e espectro de Walsh-Fourier $S_{\Omega}^{(w)}(\lambda) ;$

$\epsilon_{n}(t)$ : séries temporais estacionárias, independentes com média zero e não correlacionadas com $\Omega(t)$, funções de autocovariância $R_{\epsilon \epsilon}(\tau)$ e covariância lógica $\Gamma_{\epsilon \epsilon}(j)$ e espectro de Walsh-Fourier $S_{\epsilon}^{(w)}(\lambda)$; 
para $\tau=0, \pm 1, \cdots, 1 \leq j<T$ e $0 \leq \lambda \leq 1$. Além disso, assumimos que $R_{\Omega \Omega}(\tau)$ e $R_{\epsilon \epsilon}(\tau)$ satisfazem (3.30) e cada combinação linear de $\Omega(t)$ e $\epsilon_{n}(t)$ satisfaz (2.22) e (3.32). Assim, de acordo com o modelo (5.6), temos

(i) $E X_{n}(t)=\Theta_{n}$;

(ii) $\Gamma_{X_{n} X_{n}}(j)=\Gamma_{\Omega \Omega}(j)+\Gamma_{\epsilon \epsilon}(j)$;

(iii) $S_{X_{n} X_{n}}^{(w)}(\lambda)=S_{\Omega}^{(w)}(\lambda)+S_{\epsilon}^{(w)}(\lambda)$;

(iv) $\Gamma_{X_{q} X_{l}}=\Gamma_{\Omega \Omega}(j), q \neq l \mathrm{e}$

(v) $S_{X_{q} X_{l}}^{(w)}(\lambda)=S_{\Omega}^{(w)}(\lambda), q \neq l$.

Teorema 5.1: $\operatorname{Se}\left\{X_{n}(t), n=1, \cdots, N, t=0,1, \cdots, T-1\right\}$ satisfaz as condições do modelo (5.6), então a transformada finita de Walsh-Fourier

$$
d_{X, n}^{(T, w)}(k)=T^{-1 / 2} \sum_{t=0}^{T-1} X_{n}(t) W\left(t, \frac{k}{T}\right)
$$

tem a representação

$$
d_{X, n}^{(T, w)}(k)=U(k)+Z_{n}(k)+O_{a . s .}(1)
$$

para $k=0, \cdots, T-1$, onde $U(k)$ são variáveis independentes $N\left(0, S_{\Omega}^{(w)}\left(\frac{k}{T}\right)\right)$ e $Z_{n}(k)$ são variáveis independentes $N\left(0, S_{\epsilon}^{(w)}\left(\frac{k}{T}\right)\right)$. Além disso, $U(k)$ e $Z_{n}(k)$ são mutuamente independentes e $O_{\text {a.s. }}(1) \rightarrow 0$ quase certamente quando $T \rightarrow \infty$.

Demonstração, ver Stoffer(1987).

Usando a representação (5.8), podemos detectar se existe ou não um sinal comum, testando a hipótese $\Omega(t)=0$ para todo $t$, ou equivalentemente,

$$
H_{01}: S_{\Omega}^{(w)}(\lambda)=0 .
$$

Neste caso, nosso problema é reduzido a uma análise de variância (ANOVA) com um modelo de efeitos aleatórios (Scheffé, 1959) onde os dados são as transformadas de Walsh-Fourier das séries temporais $X_{n}(t), t=$ $0,1, \cdots, T-1$. 
Para fazer a análise consideraremos as seguintes somas de quadrados:

$$
S Q H_{01}(\lambda)=N\left[d_{X, *}^{(T, w)}(k)\right]^{2}
$$

$$
S Q R_{01}(\lambda)=\sum_{n=1}^{N}\left[d_{X, n}^{(T, w)}(k)-d_{X, \cdot}^{(T, w)}(k)\right]^{2}
$$

onde $d_{X, \cdot,}^{(T, w)}(k)=\frac{\sum_{n=1}^{N} d_{X, n}^{(T, w)}(k)}{N}$ é uma média amostral.

Utilizando o teorema de Cochran e o modelo (5.8), (5.10) e (5.11) convergem quase certamente para

$$
\left[N S_{\Omega}^{(w)}(k / T)+S_{\epsilon}^{(w)}(k / T)\right] \chi_{1}^{2}
$$

$\mathrm{e}$

$$
S_{\epsilon}^{(w)}(k / T) \chi_{N-1}^{2} .
$$

Além disso, as variáveis $\chi_{\nu}^{2}$ são independentes.

Os resultados obtidos podem ser resumidos pela Tabela 5.1: ANOVAW (análise de variância usando Walsh-Fourier).

O teste para (5.9) pode ser aplicado utilizando a estatística

$$
F_{01}(\lambda)=\frac{S Q H_{01}(\lambda) / 1}{S Q R_{01}(\lambda) /(N-1)}
$$

que, sob $H_{01}$, tem uma distribuição $\mathrm{F}(1, N-1)$.

Podemos estender a análise preliminar, com o objetivo de verificar se existe diferença entre as $L$ filas de $M$ bancos distintos. Suponha, agora, que $X_{l m}(t), l=1, \cdots, L, m=1, \cdots, M, N=L M, t=0, \cdots, T-1$, é o número de indivíduos na l-ésima fila do banco $m$, no instante $t$, e que pode ser modelado por

$$
X_{l m}(t)=\Theta_{l m}+\Omega_{m}(t)+\epsilon_{l m}(t)
$$

onde

$\Theta_{l m}=E\left(X_{l m}(t)\right)$, constante para todo $t ;$ 
Tabela 5.1: Tabela ANOVAW para teste (5.9)

\begin{tabular}{lcc}
\hline \hline fonte & soma de quadrados & grau de liberdade \\
& $(S Q)$ & $(g l)$ \\
\hline$\Omega(t)$ & $N\left[d_{X, \cdot}^{(T, w)}(k)\right]^{2}$ & 1 \\
resíduo & $\sum_{n=1}^{N}\left[d_{X, n}^{(T, w)}(k)-d_{X, \cdot}^{(T, w)}(k)\right]^{2}$ & $\mathrm{~N}-1$ \\
\hline total & $\sum_{n=1}^{N}\left[d_{X, n}^{(T, w)}(k)\right]^{2}$ & $\mathrm{~N}$ \\
\hline \hline
\end{tabular}

\begin{tabular}{|c|c|c|c|}
\hline fonte & $\begin{array}{c}\text { quadrado médio }=S Q / g l \\
(Q M)\end{array}$ & $\begin{array}{c}\text { estatística } \\
F\end{array}$ & $\begin{array}{c}\text { quadrado médio esperado } \\
\text { (EQM) }\end{array}$ \\
\hline$\Omega(t)$ & $N\left[d_{X, \cdot}^{(T, w)}(k)\right]^{2}(\star)$ & $(\star) /(\star \star)$ & $N S_{\Omega}^{(w)}\left(\frac{k}{T}\right)+S_{\epsilon}^{(w)}\left(\frac{k}{T}\right)$ \\
\hline resíduo & $\frac{\sum_{n=1}^{N}\left[d_{X, n}^{(T, w)}(k)-d_{X, *}^{(T, w)}(k)\right]^{2}}{N-1}(\star \star)$ & & $S_{\epsilon}^{(w)}\left(\frac{k}{T}\right)$ \\
\hline
\end{tabular}

$\Omega_{m}(t)$ : processos estacionários com média zero e espectros de Walsh-Fourier $S_{\Omega m}^{(w)}(\lambda) ;$

$\epsilon_{l m}(t)$ : processos estacionários independentes com média zero e espectro de Walsh-Fourier comum $S_{\epsilon}^{(w)}(\lambda)$, tal que $\Omega_{m}(t)$ e $\epsilon_{l m}(t)$ são não correlacionados e $\epsilon_{i h}(t)$ e $\epsilon_{j k}(t)$ são mutuamente independentes, $i, j=1, \cdots, L$, $h \neq k$.

Denotando $d_{X, l m}^{(T, w)}(k)$ a transformada de Walsh-Fourier de $X_{l m}(t)$, na sequência $\frac{k}{T}, k=1, \cdots, T-1$, sob as condições apropriadas do modelo (5.15), para $\frac{k}{T}$ fixo, temos a seguinte representação

$$
d_{X, l m}^{(T, w)}(k)=U_{m}(k)+Z_{l m}(k)+O_{a . s .}(1),
$$


onde para cada $k, U_{m}(k)$ são variáveis $N\left(0, S_{\Omega m}^{(w)}\left(\frac{k}{T}\right)\right)$ independentes, $Z_{l m}(k)$ são variáveis $N\left(0, S_{c}^{(w)}\left(\frac{k}{T}\right)\right)$ independentes e independentes de $U(k)$. Além disso, $O_{\text {a.s. }}(1) \rightarrow 0$ quase certamente quando $T \rightarrow \infty$.

Nosso problema é detectar se existe diferença entre os sinais $\Omega_{1}(t), \Omega_{2}(t)$, $\cdots, \Omega_{M}(t)$. Para isso, $\Omega_{m}(t)$ é decomposto da seguinte forma

$$
\Omega_{m}(t)=\mu(t)+\alpha_{m}(t),
$$

para $m=1, \cdots, M$, onde

$\mu(t)$ : sinal comum que tem espectro de Walsh-Fourier $S_{\mu}^{(w)}(\lambda)$;

$\alpha_{m}(t)$ : sinal particular para o grupo $\mathrm{m}$, independentes e com espectro de Walsh-Fourier comum $S_{\Omega}^{(w)}(\lambda)$.

Dessa forma,

$$
S_{\Omega m}^{(w)}(\lambda)=S_{\mu}^{(w)}(\lambda)+S_{\Omega}^{(w)}(\lambda)
$$

e (5.16) pode ser reescrito da seguinte forma

$$
d_{X, l m}^{(T, w)}(k)=U(k)+V_{m}(k)+Z_{l m}(k)+O_{a . s .}(1)
$$

onde para cada $k, U, V$ e $Z$ são variáveis independentes com distribuições $N\left(0, S_{\mu}^{(w)}\left(\frac{k}{T}\right)\right), N\left(0, S_{\Omega}^{(w)}\left(\frac{k}{T}\right)\right)$ e $N\left(0, S_{\epsilon}^{(w)}\left(\frac{k}{T}\right)\right)$, respectivamente. As comparações dos sinais dos grupos é realizada pelo teste

$$
H_{02}: \alpha_{1}(t)=\alpha_{2}(t)=\cdots=\alpha_{M}(t)=0,
$$

para todo $\mathrm{t}$, ou equivalentemente,

$$
H_{03}: S_{\Omega}^{(w)}(\lambda)=0,0<\lambda<1 .
$$

Para fazer o teste consideraremos as seguintes somas de quadrados

$$
S Q H_{03}(\lambda)=L \sum_{m=1}^{M}\left[d_{X, \cdot m}^{(T, w)}(k)-d_{X, \cdot *}^{(T, w)}(k)\right]^{2}
$$

$\mathrm{e}$

$$
S Q R_{03}(\lambda)=\sum_{m=1}^{M} \sum_{l=1}^{L}\left[d_{X, l m}^{(T, w)}(k)-d_{X, \cdot m}^{(T, w)}(k)\right]^{2},
$$


onde

$$
\begin{gathered}
d_{X, \cdot m}^{(T, w)}(k)=L^{-1} \sum_{l=1}^{L} d_{X, l m}^{(T, w)}(k), \\
d_{X, \cdot \bullet}^{(T, w)}(k)=M^{-1} \sum_{m=1}^{M} d_{X, \cdot m}^{(T, w)}(k) .
\end{gathered}
$$

Devido do modelo (5.19), (5.22) e (5.23) convergem quase certamente para

e

$$
\left[L S_{\Omega}^{(w)}\left(\frac{k}{T}\right)+S_{\epsilon}^{(w)}\left(\frac{k}{T}\right)\right] \chi_{M-1}^{2}
$$

$$
S_{\epsilon}^{(w)}\left(\frac{k}{T}\right) \chi_{(N-M)}^{2}
$$

onde $\chi_{\nu}^{2}$ são independentes.

Os resultados obtidos podem ser resumidos pela Tabela (5.2).

Tabela 5.2: Tabela ANOVAW para teste (5.20)

\begin{tabular}{lcc}
\hline \hline fonte & soma de quadrados & grau de liberdade \\
& $(S Q)$ & $(g l)$ \\
\hline grupos & $L \sum_{m=1}^{M}\left[d_{X, \cdot m}^{(T, w)}(k)-d_{X, \cdot *}^{(T, w)}(k)\right]^{2}$ & $\mathrm{M}-1$ \\
resíduo & $\sum_{m=1}^{M} \sum_{l=1}^{L}\left[d_{X, l m}^{(T, w)}(k)-d_{X, \cdot m}^{(T, w)}(k)\right]^{2}$ & $\mathrm{~N}-\mathrm{M}$ \\
\hline total & $\sum_{m=1}^{M} \sum_{l=1}^{L}\left[d_{X, l m}^{(T, w)}(k)-d_{X, \cdot *}^{(T, w)}(k)\right]^{2}$ & $\mathrm{~N}-1$ \\
\hline \hline
\end{tabular}

\begin{tabular}{lccc}
\hline \hline fonte & quadrado médio=SQ/gl & estatística & quadrado médio esperado \\
& $(Q M)$ & $F$ & $(E Q M)$ \\
\hline grupos & $\frac{L \sum_{m=1}^{M}\left[d_{X,-m}^{(T, w)}(k)-d_{X, *}^{(T, w)}(k)\right]^{2}}{M-1}(\star)$ & $(\star) /(\star \star)$ & $L S_{\Omega}^{(w)}\left(\frac{k}{T}\right)+S_{\epsilon}^{(w)}\left(\frac{k}{T}\right)$ \\
resíduo & $\frac{\sum_{m=1}^{M} \sum_{\ell=1}^{L}\left[d_{X, i m}^{(T, w)}(k)-d_{X,-m}^{(T, w)}(k)\right]^{2}}{(N-M)}(\star \star)$ & & $S_{\epsilon}^{(w)}\left(\frac{k}{T}\right)$ \\
\hline total & & \\
\hline \hline
\end{tabular}


O teste para (5.21) é realizado utilizando a estatística

$$
F_{03}(\lambda)=\frac{S Q H_{03}(\lambda) /(M-1)}{S Q R_{03}(\lambda) /(N-M)}
$$

que, sob $H_{03}$, tem uma distribuição $\mathrm{F}(M-1, N-M)$ para $k=1, \cdots, T-1$ e $\lambda=\frac{k}{T}$.

Os testes baseados em $F(\lambda)$ são apenas apropriados para uma frequência predeterminada. Brillinger(1980) e Shumway(1988) sugerem que o problema de se fazer afirmaçôes simultâneas, sobre o valor do espectro, pode ser contornado utilizando uma correção de Bonferroni. Ver Stoffer et al(1988).

\subsection{Séries temporais categóricas}

Quando utilizamos técnicas estatísticas quantitativas em variáveis qualitativas, "scaling" fornece um método de atribuir valores numéricos para as categorias conforme alguns critérios especificados. Por exemplo, ao EEG dos seis possiveis estados de sono de uma criança, mostrado na Figura 5.2, são atribuídos arbitrariamente os valores de 1 a 6 da seguinte forma: estado 1: "quiet sleep - trace alternant"; estado 2: "quiet sleep - high voltage"; estado 3: "indeterminate sleep"; estado 4: "active sleep"; estado 5: "active sleep - mixed" e estado 6: " awake". Entretanto, podemos quantificar essas categorias, segundo alguns critérios de otimização, e fazer, de uma maneira mais adequada, uma análise espectral e uma análise de variância quando for conveniente.

Seja $X^{*}(t)$ o valor categórico de uma série, e $Y(t)=\left(Y_{1}(t), \cdots, Y_{J}(t)\right)^{\prime}$ o vetor aleatório tal que

$$
Y_{j}(t)= \begin{cases}1 & \text { se } X^{*}(t) \text { está no estado } j \text { no instante } t \\ 0 & \text { caso contrário, }\end{cases}
$$

onde $j=1, \cdots, J$ e $J$ é o número de possíveis estados ou categorias. Por exemplo, a série do EEG durante o estado de sono de uma criança, Figura 5.2 , exibe seis possíveis estados ou categorias, portanto, neste caso, $J=6$. A Figura 5.8, mostra um EEG durante o estado de sono de uma criança cuja mãe consumiu bebidas alcoólicas durante sua gravidez; a série durante o 
estado de sono é "indeterminate", "mixed active", "indeterminate", "mixed active", $\cdots$, então, nesse caso,

$$
\begin{aligned}
& Y(0)=(0,0,1,0,0,0)^{\prime} \\
& Y(1)=(0,0,0,0,1,0)^{\prime} \\
& Y(2)=(0,0,1,0,0,0)^{\prime} \\
& Y(3)=(0,0,0,0,1,0)^{\prime}
\end{aligned}
$$

"Scaling" consiste em atribuir valores numéricos para as categorias de $X^{*}(t)$ segundo algum critério ótimo. Esse problema pode ser visto como uma seleção ótima de um vetor de dimensão $J \times 1$ de números reais, $\beta=$ $\left(\beta_{1}, \cdots, \beta_{J}\right)^{\prime}$, para produzir uma série temporal univariada

$$
X(t)=\beta^{\prime} Y(t)=\sum_{j=1}^{J} \beta_{j} Y_{j}(t)
$$

que possa ser analisada utilizando as técnicas convencionais de série temporal. Pela definição dos $Y_{j}(t)$, se o processo categórico está no estado $j$, no instante $t$, então $X(t)=\beta_{j}$.

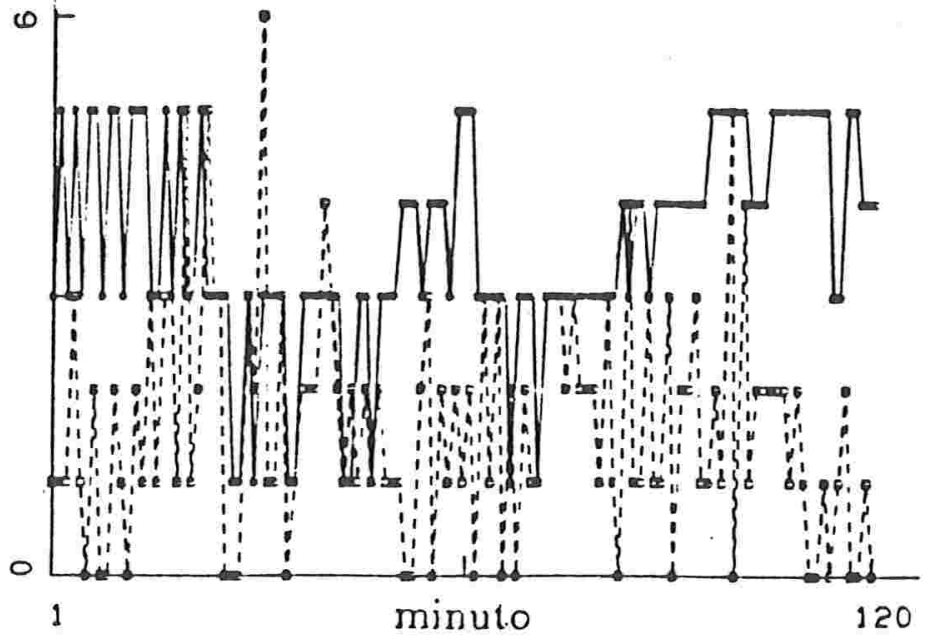

Figura 5.8: EEG durante o estado de sono de uma criança cuja mãe consumiu bebidas alcoólicas durante sua gravidez 
Análogo ao modelo (5.6), podemos modelar $Y_{n}(t)$ por

$$
Y_{n}(t)=\Theta_{n}+\Omega(t)+\epsilon_{n}(t),
$$

cuja matriz espectral, de dimensão $J \times J$ é

$$
S_{Y_{n}}(\lambda)=S_{\Omega}(\lambda)+S_{\epsilon}(\lambda),
$$

$0<\lambda<1$ e, portanto, o espectro da série temporal univariada $X_{n}(t)=$ $\beta^{\prime} Y_{n}(t)$ é

$$
S_{X_{n}}(\lambda)=\beta^{\prime} S_{\Omega}(\lambda) \beta+\beta^{\prime} S_{\epsilon}(\lambda) \beta \text {. }
$$

Pode ser demonstrado que o parâmetro de não-centralidade do teste dado em (5.14) é essencialmente a "razão sinal-ruído" do processo de $X_{n}(t)$, isto é,

$$
\frac{\beta^{\prime} S_{\Omega}(\lambda) \beta}{\beta^{\prime} S_{\epsilon}(\lambda) \beta} .
$$

Portanto, o vetor $\beta$ pode ser escolhido de forma a maximizar (5.32). Como $S_{\Omega}(\lambda)$ e $S_{\epsilon}(\lambda)$ são desconhecidos, o problema se transforma na escolha do vetor $\beta$ de forma a maximizar

$$
\frac{\beta^{\prime} \widehat{S}_{\Omega}(\lambda) \beta}{\beta^{\prime} \widehat{S}_{\epsilon}(\lambda) \beta}
$$

onde $\widehat{S}_{\Omega}(\lambda)$ e $\widehat{S}_{\epsilon}(\lambda)$ são estimadores consistentes de $S_{\Omega}(\lambda)$ e $S_{\epsilon}(\lambda)$, respectivamente.

Por exemplo, podemos escolher $\beta$ para maximizar (5.33) numa particular sequência de interesse, ou para maximizar

$$
\frac{\beta \bar{S}_{\Omega} \beta}{\beta^{\prime} \bar{S}_{\epsilon} \beta}
$$

onde

$$
\bar{S}_{\Omega}=\sum_{k=1}^{T-1} \widehat{S}_{\Omega}\left(\frac{k}{T}\right)
$$

$$
\bar{S}_{\epsilon}=\sum_{k=1}^{T-1} \widehat{S}_{\epsilon}\left(\frac{k}{T}\right),
$$


ou, também,

$$
\bar{S}_{\Omega}=\sum_{k=1}^{T-1} \operatorname{tr}\left\{\widehat{S}_{\Omega}\left(\frac{k}{T}\right)\right\} \widehat{S}_{\Omega}\left(\frac{k}{T}\right)
$$

$\mathrm{e}$

$$
\bar{S}_{\epsilon}=\sum_{k=1}^{T-1} \operatorname{tr}\left\{\widehat{S}_{\epsilon}\left(\frac{k}{T}\right)\right\} \widehat{S}_{\epsilon}\left(\frac{k}{T}\right)
$$

onde $\operatorname{tr}$ denota traço de uma matriz. Nesses casos, o vetor $\beta$ ótimo pode ser obtido pelo auto-vetor de (5.34).

Para mais detalhes, ver Stoffer(1987, 1990, 1991) e Rao(1973).

Um procedimento para obter o vetor $\beta$ ótimo de cada grupo é dado por:

(a) construir o vetor $Y_{n}(t)$ de dimensão $J \times 1$ para cada elemento $n$ do grupo;

(b) calcular a transformada de Walsh-Fourier $d_{Y, n}^{(T, w)}(k)$ dos vetores $Y_{n}(t)$, $n=1, \cdots, N$, usando a transformada rápida de Walsh-Fourier;

(c) calcular a transformada média de Walsh-Fourier

$$
d_{Y, \cdot}^{(T, w)}(k)=N^{-1} \sum_{n=1}^{N} d_{Y, n}^{(T, w)}(k)
$$

(d) estimar a matriz de $S_{\epsilon}\left(\frac{k}{T}\right)$, de dimensão $J \times J$, por

$$
\widehat{S}_{\epsilon}\left(\frac{k}{T}\right)=\frac{\sum_{n=1}^{N}\left[d_{Y, n}^{(T, w)}(k)-d_{\left.Y^{\prime}, w\right)}^{(T, w)}(k)\right]\left[d_{Y, n}^{(T, w)}(k)-d_{Y^{\prime}, \cdot}^{(T, w)}(k)\right]^{\prime}}{N-1},
$$

e a matriz de $S_{\Omega}\left(\frac{k}{T}\right)$, de dimensão $J \times J$, por

$$
\widehat{S}_{\Omega}\left(\frac{k}{T}\right)=N\left[d_{Y, \cdot}^{(T, w)}(k)\right]\left[d_{Y, \cdot}^{(T, w)}(k)\right]^{\prime} ;
$$

(e) calcular

$$
\bar{S}_{\epsilon}=\sum_{k=1}^{T-1} \widehat{S}_{\epsilon}\left(\frac{k}{T}\right)
$$

$\mathrm{e}$

$$
\bar{S}_{\Omega}=\sum_{k=1}^{T-1} \widehat{S}_{\Omega}\left(\frac{k}{T}\right)
$$


(f) obter o vetor $\beta$ ótimo maximizando

$$
\frac{\beta^{\prime} \bar{S}_{\Omega} \beta}{\beta^{\prime} \bar{S}_{\epsilon} \beta}
$$

sujeito às restrições

$$
\max \left\{\frac{\beta^{\prime} \bar{S}_{\Omega} \beta}{\beta^{\prime} \bar{S}_{\epsilon} \beta}\right\}=\rho_{1}
$$

e

$$
\beta=P_{1}
$$

onde $\rho_{1}$ é o maior auto-valor de $\left(\bar{S}_{\Omega} \cdot \bar{S}_{\epsilon}^{-1}\right)$ e $P_{1}$ é o seu correspondente auto-vetor.

A obtenção do vetor $\beta$ ótimo para fazer comparação entre os grupos, utilizando a estatística (5.28), é feita seguindo os procedimentos (a)-(f) com

$$
\widehat{S}_{c}\left(\frac{k}{T}\right)=\frac{\sum_{m=1}^{M} \sum_{l=1}^{L}\left[d_{Y, l m}^{(T, w)}(k)-d_{Y, \cdot m}^{(T, w)}(k)\right]\left[d_{Y, l m}^{(T, w)}(k)-d_{Y, \cdot m}^{(T, w)}(k)\right]^{\prime}}{N-M}
$$

e

$$
\widehat{S}_{\Omega}\left(\frac{k}{T}\right)=\frac{L \sum_{m=1}^{M}\left[d_{Y, m}^{(T, w)}(k)-d_{Y, \cdot *}^{(T, w)}(k)\right]\left[d_{Y, \cdot m}^{(T, w)}(k)-d_{Y, *}^{(T, w)}(k)\right]^{\prime}}{M-1}
$$

onde $d_{Y, l m}^{(T, w)}(k)$ é a transformada de Walsh-Fourier do vetor $Y_{l m}(t)$ para o l-ésimo elemento $(l=1,2, \cdots, L)$ do grupo $m(m=1,2, \cdots, M)$.

Para mais detalhes, ver Stoffer(1991). 


\title{
Capítulo 6
}

\author{
Aplicações
}

\subsection{Análise de Variância utilizando a trans- formada de Fourier}

Os dados desta aplicação referem-se aos eletroscilogramas de quarenta ratos normais de três a quatro meses de idade, de ambos os sexos, com pesos compreendidos entre duzentos e duzentos e cinquenta gramas. As observações foram obtidas durante a atividade rítmica dos ratos de acordo com um modelo de planejamento cruzado com dois fatores fixos,

$$
Y_{l m q}(t)=\mu_{l m q}+\Omega(t)+\Gamma_{m}(t)+\Delta_{q}(t)+(\Gamma \Delta)_{m q}(t)+\epsilon_{l m q}(t),
$$

com os quarenta ratos subdivididos da seguinte forma:

\begin{tabular}{|c|c|c|c|}
\hline & & \multicolumn{2}{|c|}{ Região } \\
\hline & & \multicolumn{2}{|c|}{10 Hipotálamo } \\
\hline \multirow{6}{*}{ Estado } & & rato $01 \quad\left(Y_{1,1,1}\right)$ & rato $21 \quad\left(Y_{1,2,1}\right)$ \\
\hline & Alerta & (PAR) & (HAR) \\
\hline & & rato $10 \quad\left(Y_{10,1,1}\right)$ & rato $30 \quad\left(Y_{10,2,1}\right)$ \\
\hline & & rato $11 \quad\left(Y_{1,1,2}\right)$ & rato $31 \quad\left(Y_{1,2,2}\right)$ \\
\hline & Sono & $\vdots \quad$ (PSR) & $\vdots \quad \quad$ (HSR) \\
\hline & paradoxal & rato $20 \quad\left(Y_{10,1,2}\right)$ & rato $40 \quad\left(Y_{10,2,2}\right)$ \\
\hline
\end{tabular}


onde

$Y_{l m q}(t)$ é o eletroscilograma do l-ésimo rato, sujeito aos fatores região $(\mathrm{m})$ e estado (q), no instante $t, l=1, \cdots, 10, m=1,2$ e $q=1,2$;

$\mu_{l m q}$ é um valor médio dos eletroscilogramas do l-ésimo rato, sujeito aos fatores região (m) e estado (q);

$\Omega(t)$ é o sinal comum dos eletroscilogramas de todos os ratos, no instante t;

$\Gamma_{m}(t)$ é o comportamento comum dos eletroscilogramas dos ratos na mésima região (posteriotálamo ou hipotálamo), no instante t, $m=1,2$;

$\Delta_{q}(t)$ é o comportamento comum dos eletroscilogramas dos ratos durante o q-ésimo estado (alerta ou sono paradoxal), no instante t, $q=1,2$;

$(\Gamma \Delta)_{m q}(t)$ é a interação entre os fatores região e estado, no instante t;

$\epsilon_{l m q}(t)$ é o erro aleatório.

Os dados coletados estão apresentados na Figura 6.1; cada série contém 256 pontos do eletroscilograma medidos em microvolts durante 1,7 segundos. Notamos que a maioria deles se distribuem na faixa de -200 a 200 microvolts, excetuando-se os ratos do grupo HSR (rato 31 a rato 40) que apresentam alguns valores fora desse intervalo. Os periodogramas estão apresentados na Figura 6.2. Analisando cada um deles, notamos que existem valores altos em baixas frequências.

$\mathrm{Na}$ primeira etapa da análise, vamos verificar a existência de interação entre as regiões e os estados, ou seja, testar a hipótese

$$
H_{01}:(\Gamma \Delta)_{11}(t)=(\Gamma \Delta)_{12}(t)=(\Gamma \Delta)_{21}(t)=(\Gamma \Delta)_{22}(t)=0
$$

A análise é feita utilizando a Tabela 4.5 (ANOVAF). Desenvolvendo as fórmulas para um modelo com dois fatores cruzados fixos, com dois níveis cada, chegamos à Tabela 6.1 de análise de variância que deve ser utilizada neste caso. 

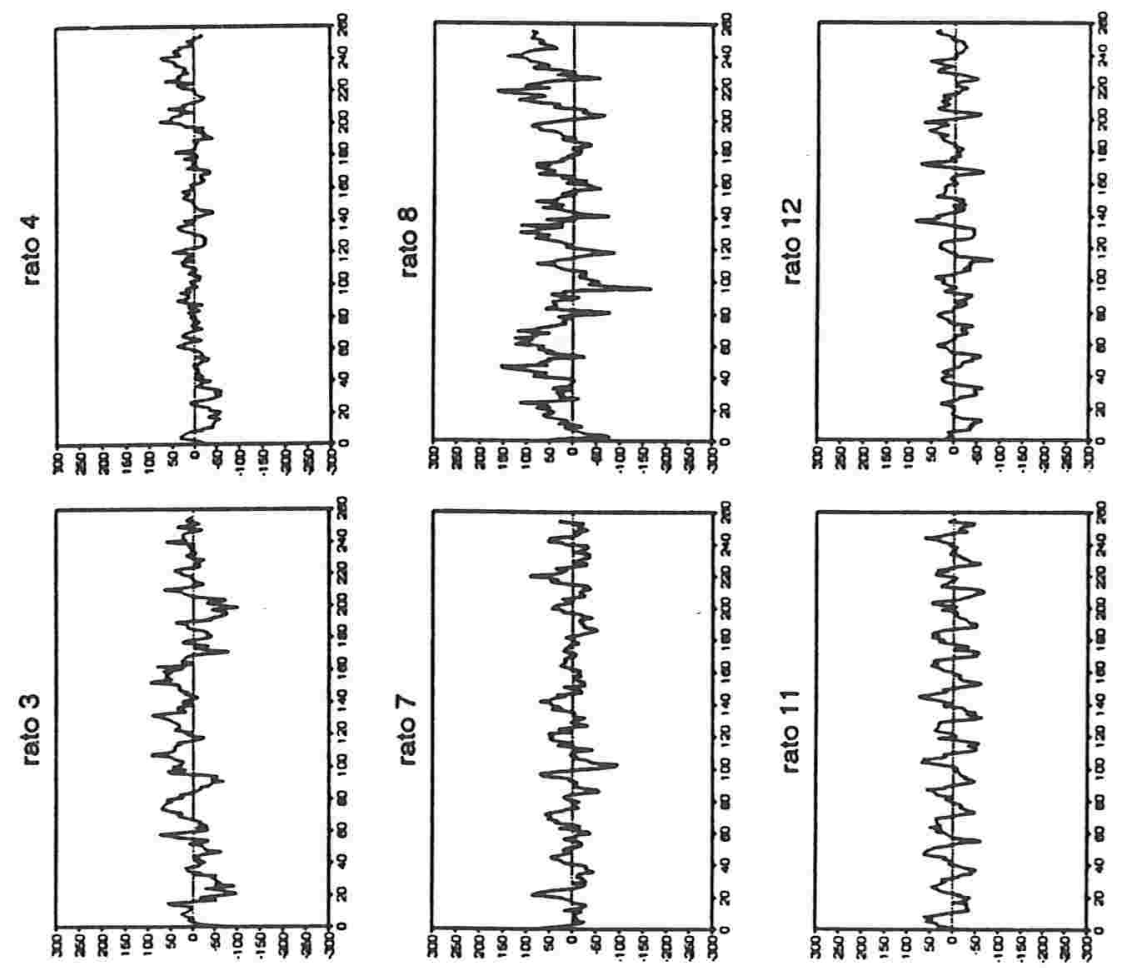

I্
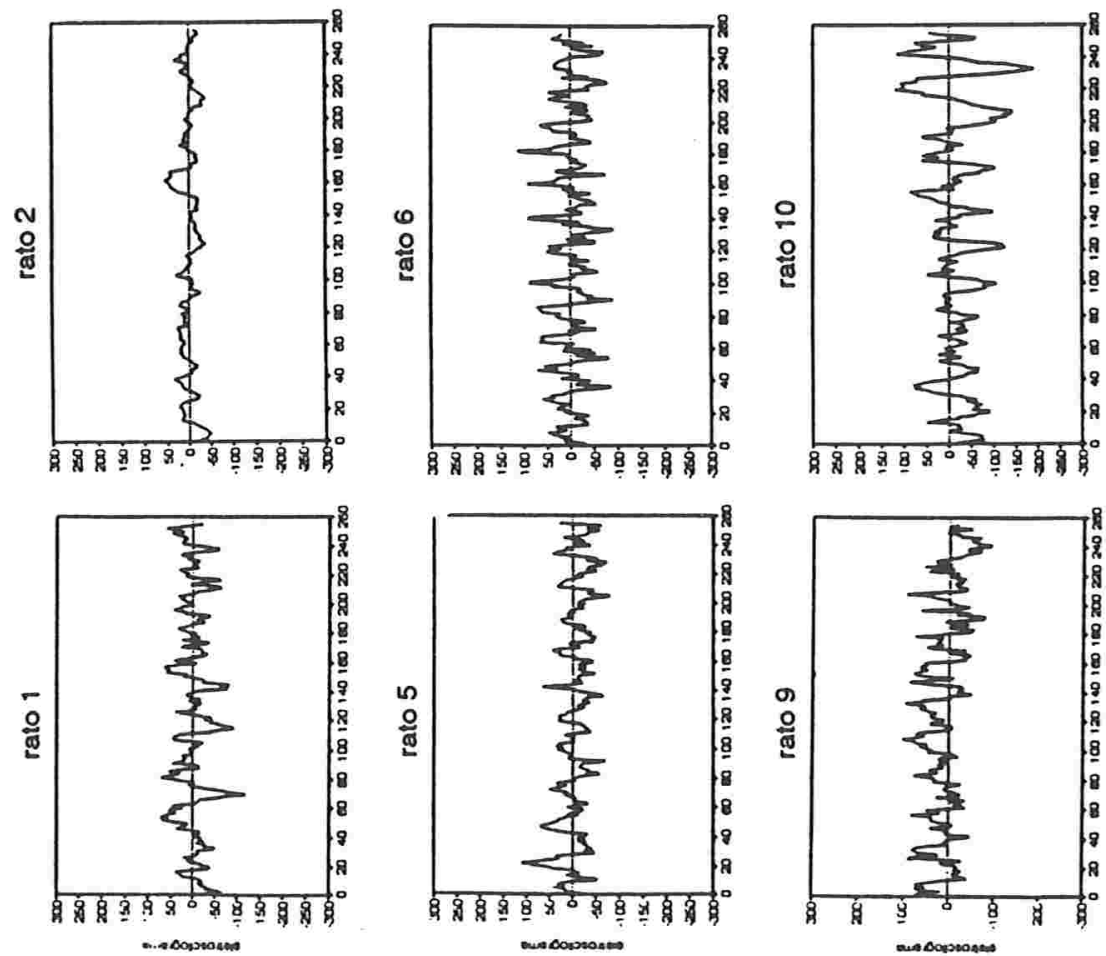

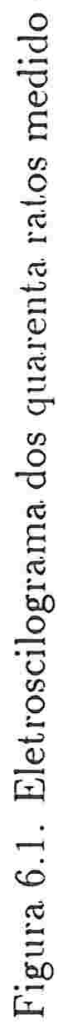



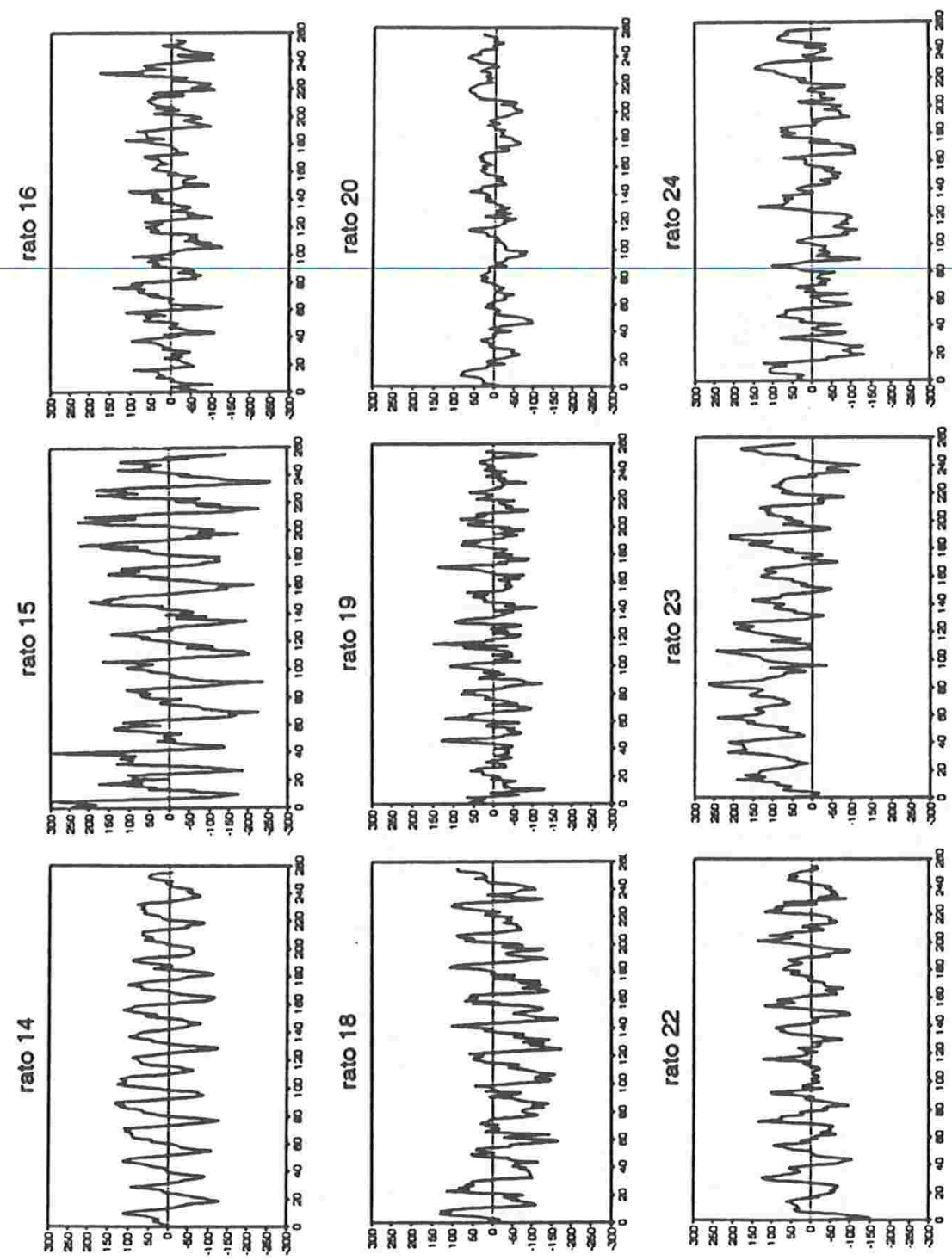

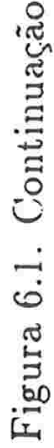
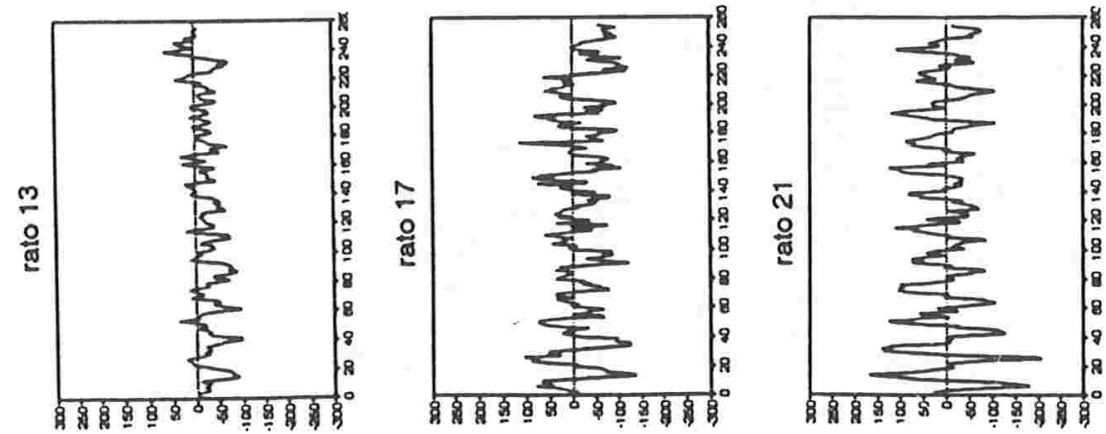

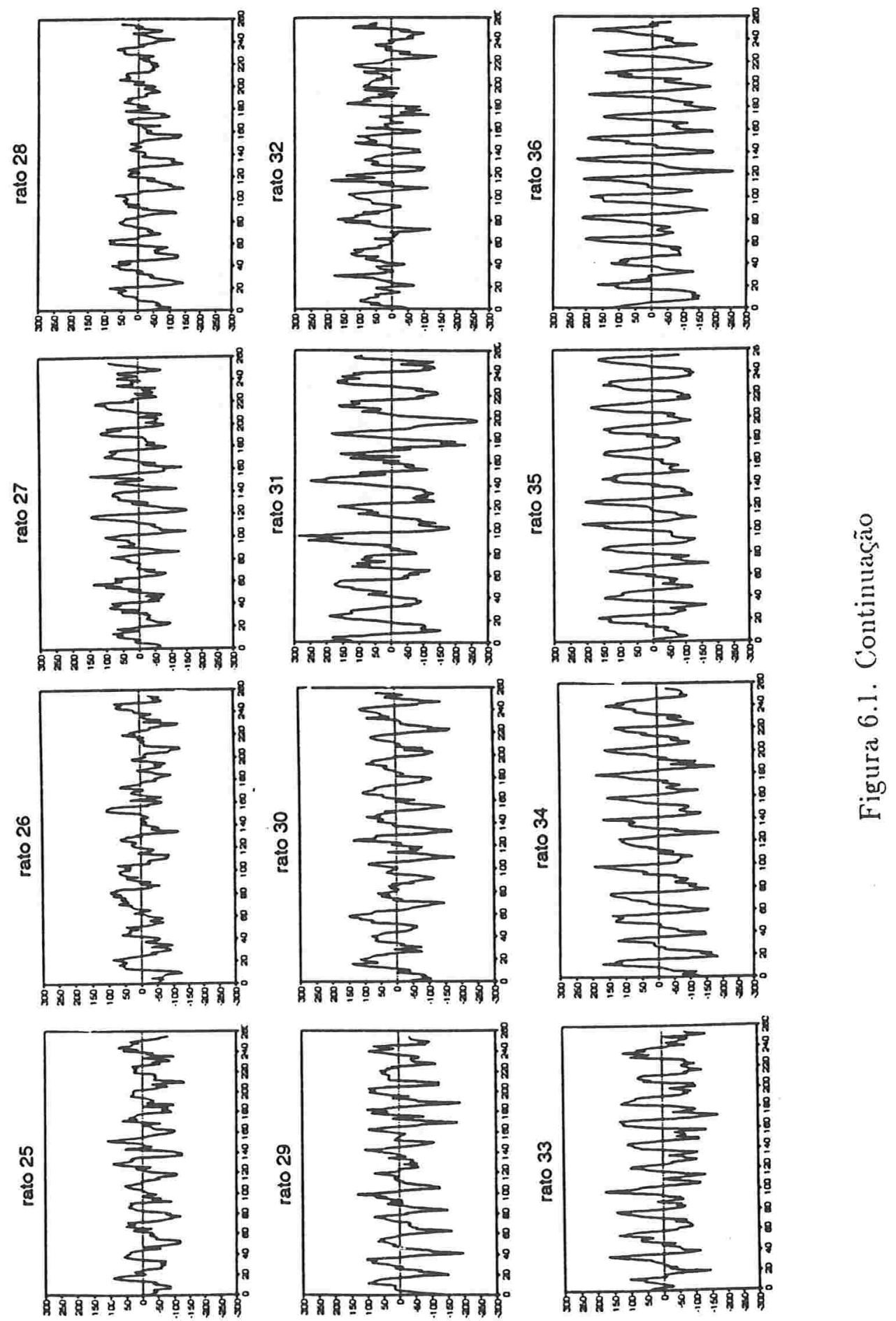

总 

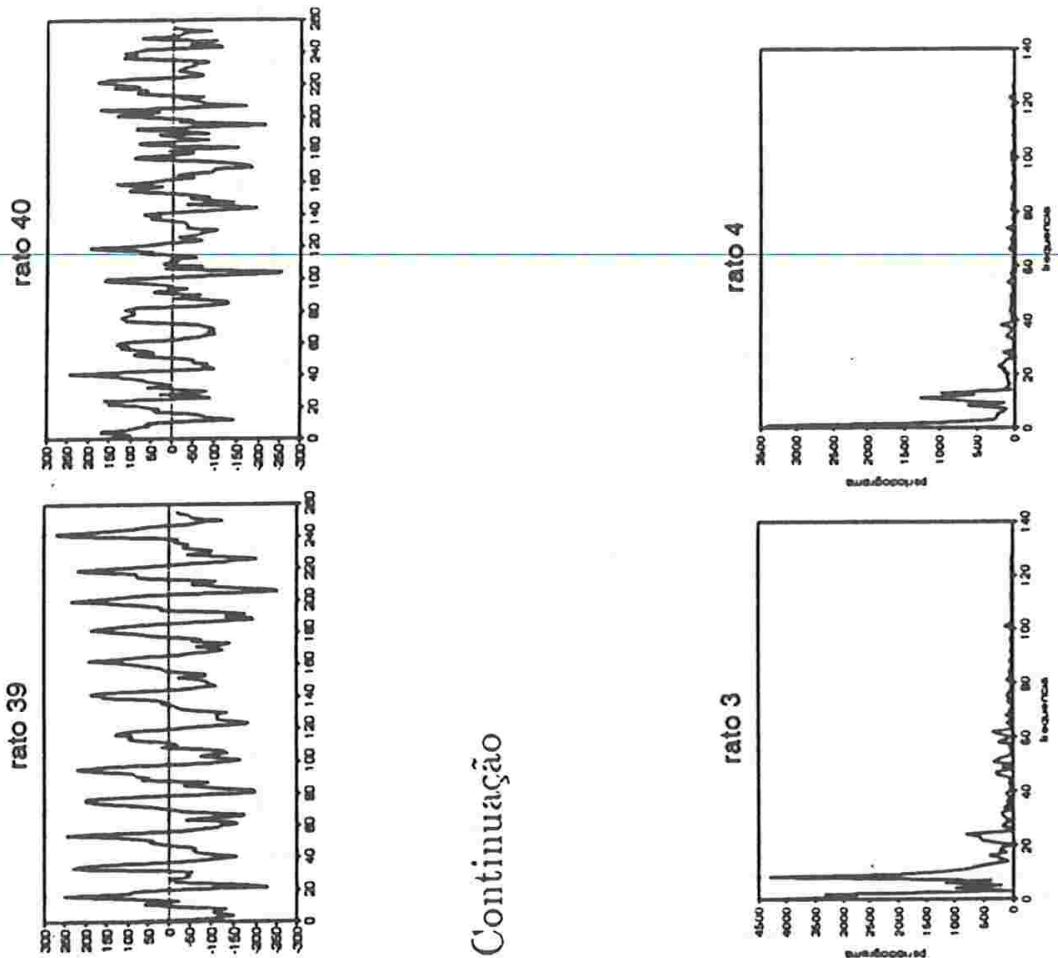

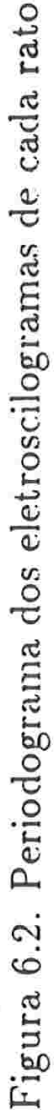

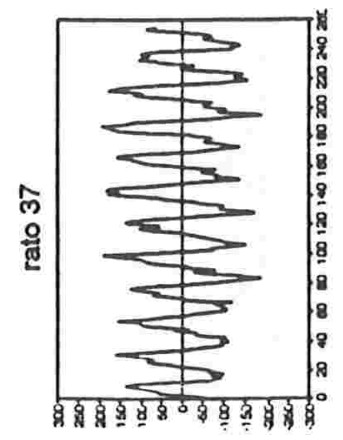

㭡
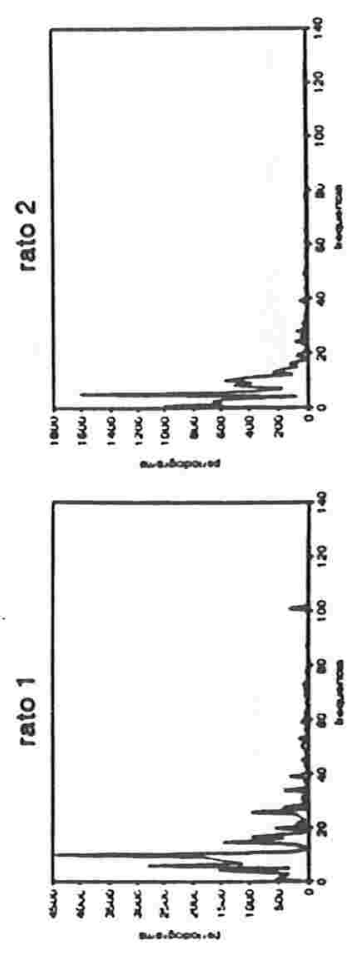

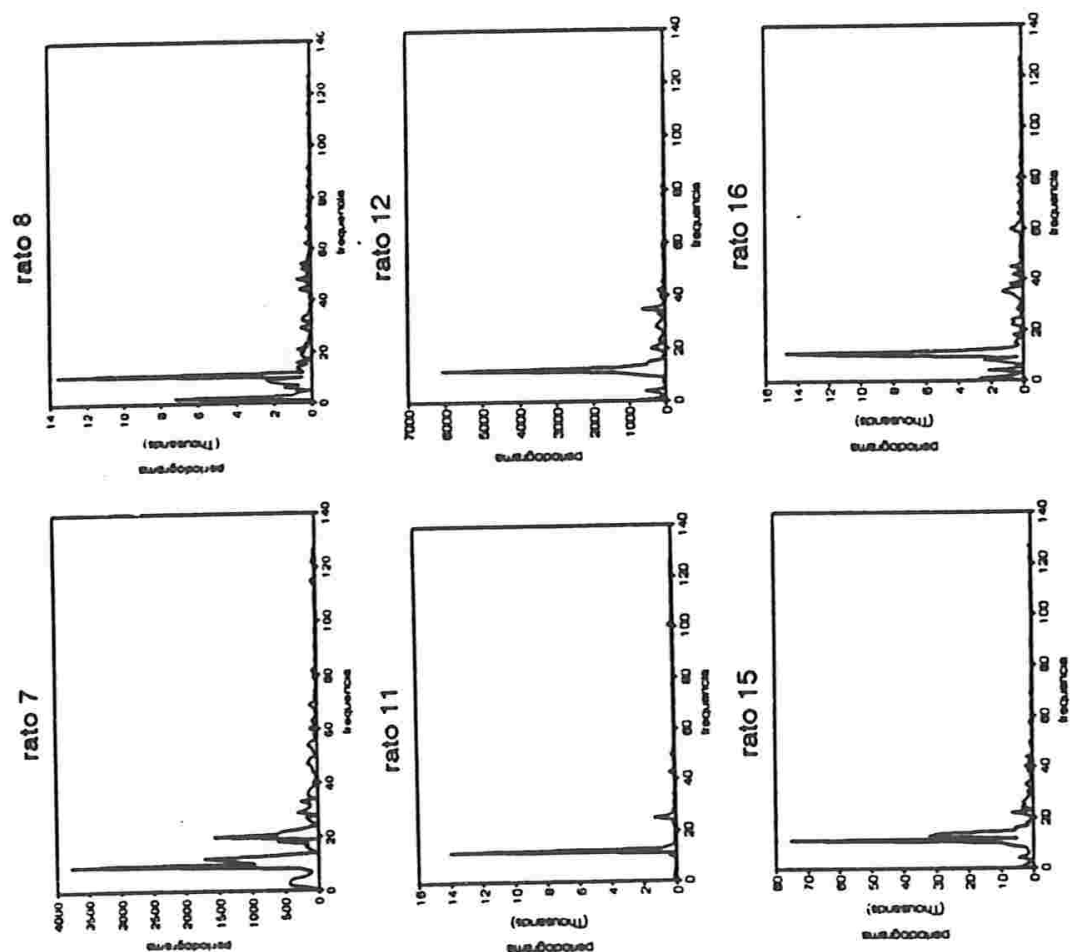

芯
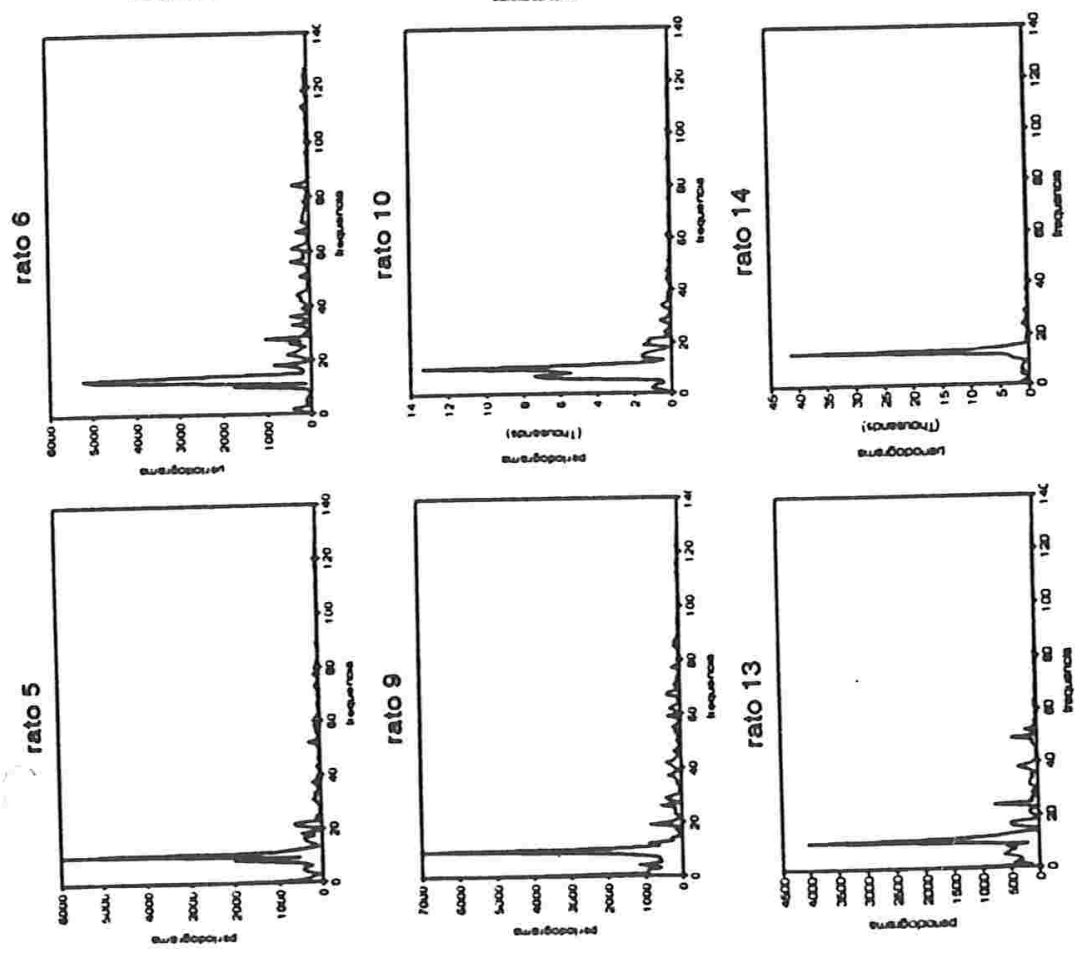

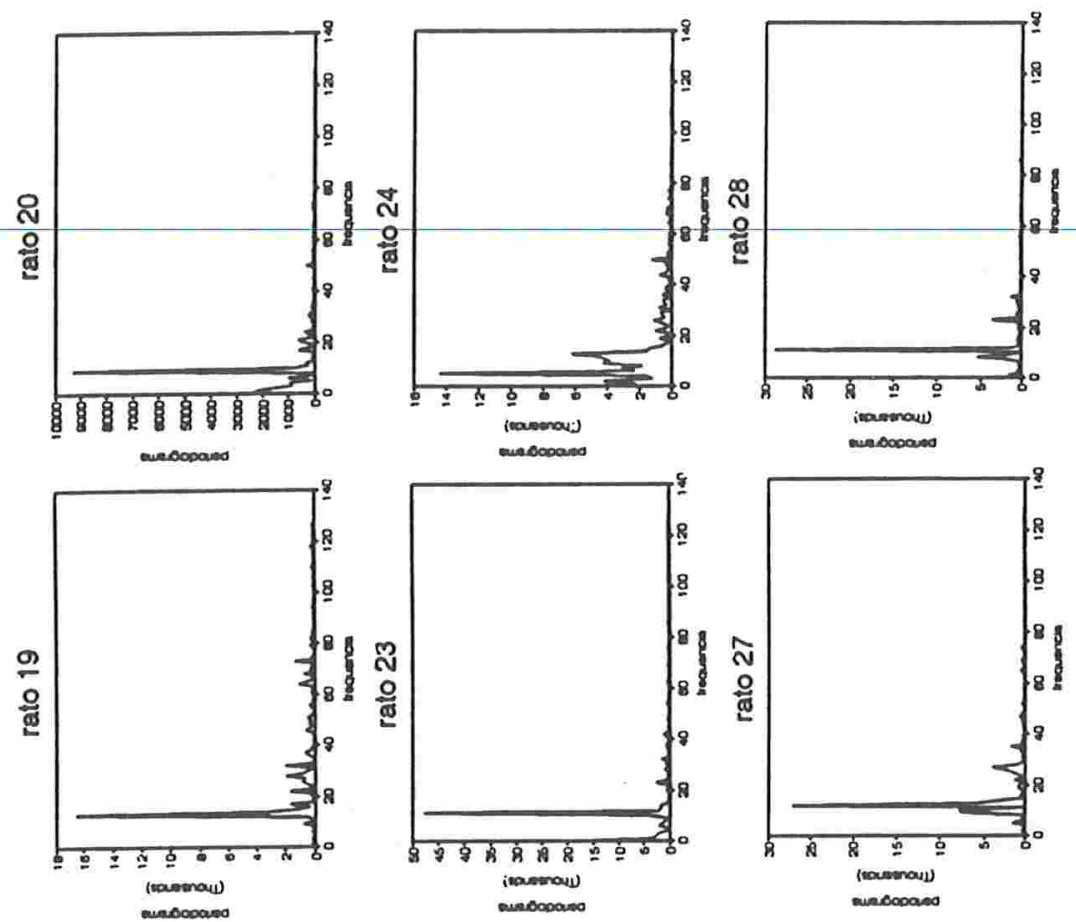


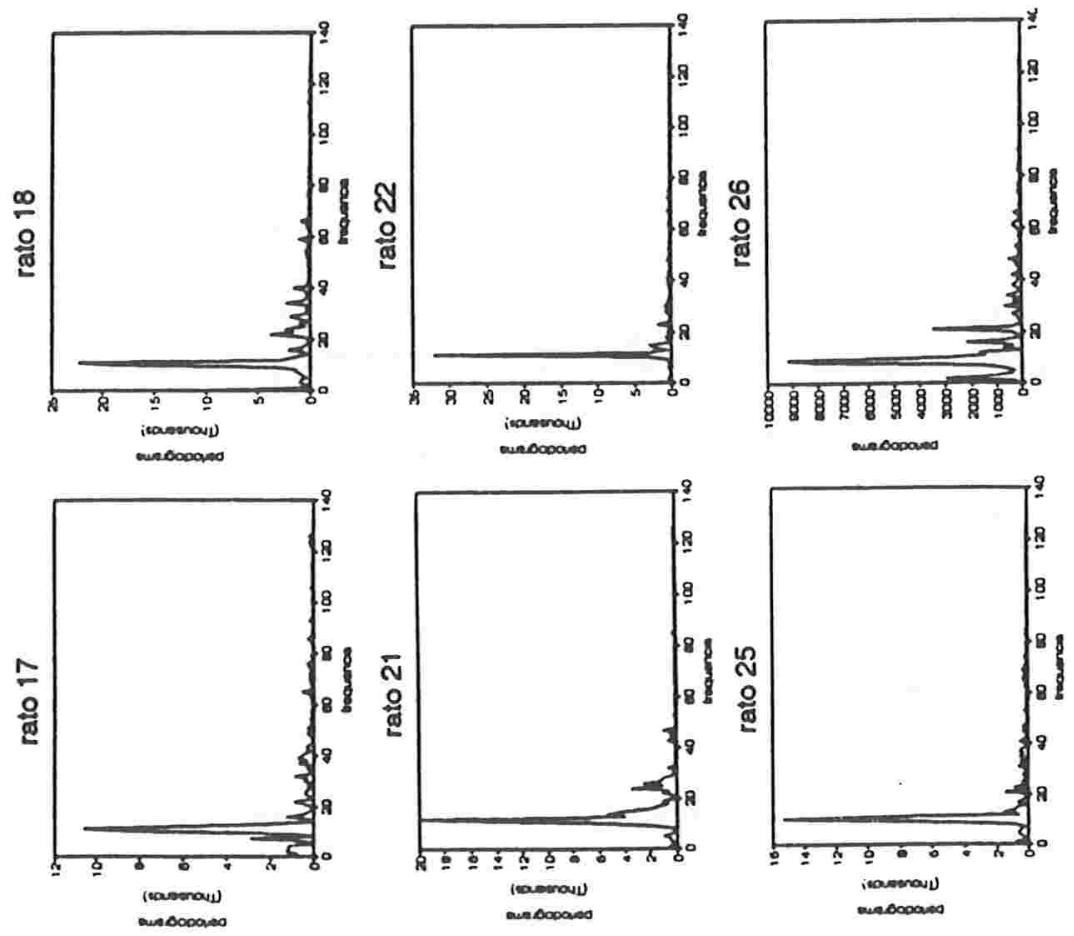

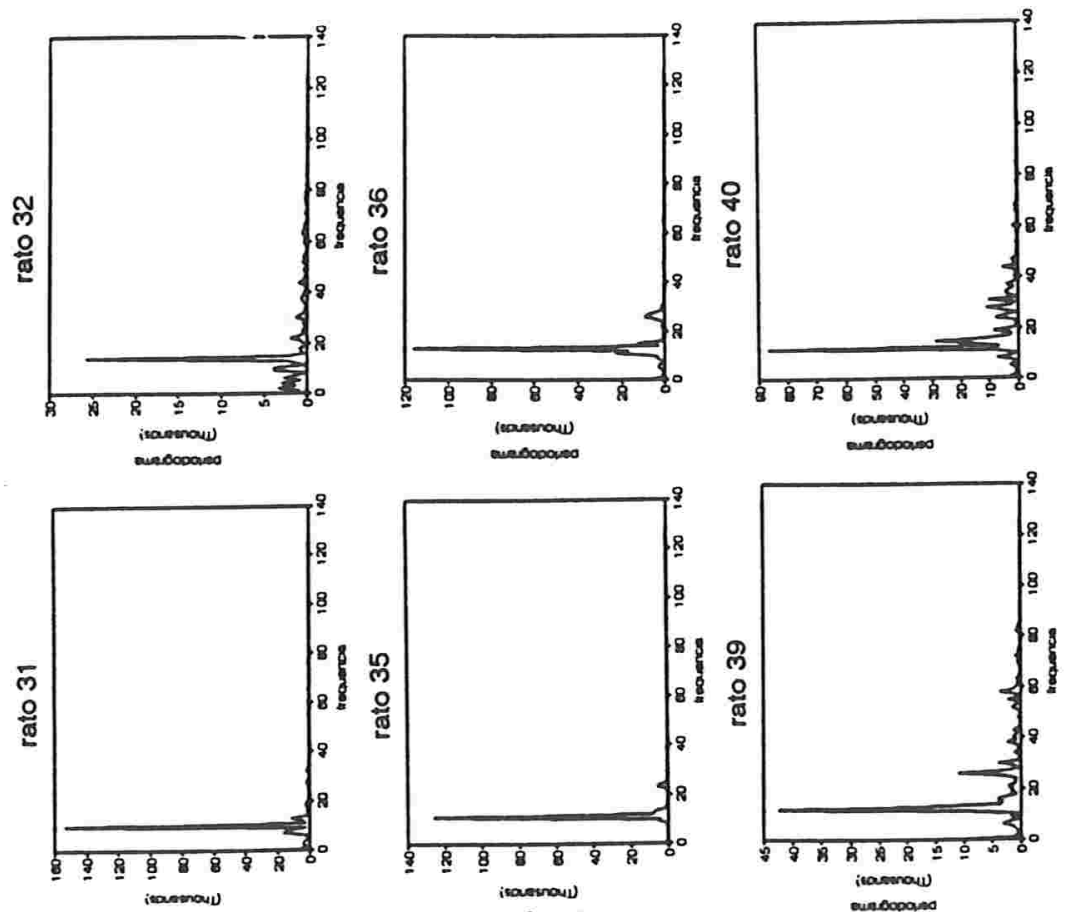

怘
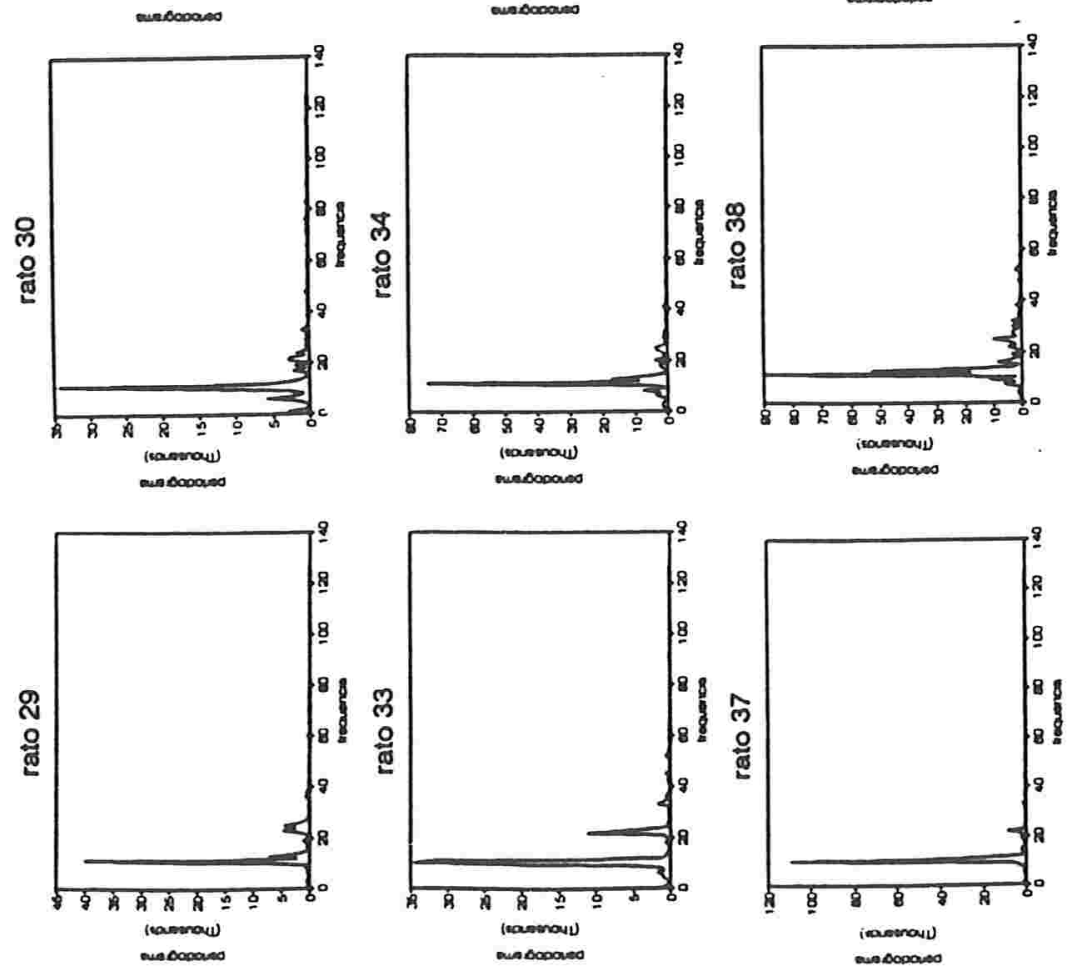
Tabela 6.1: ANOVAF para o modelo com dois fatores cruzados fixos com dois níveis cada

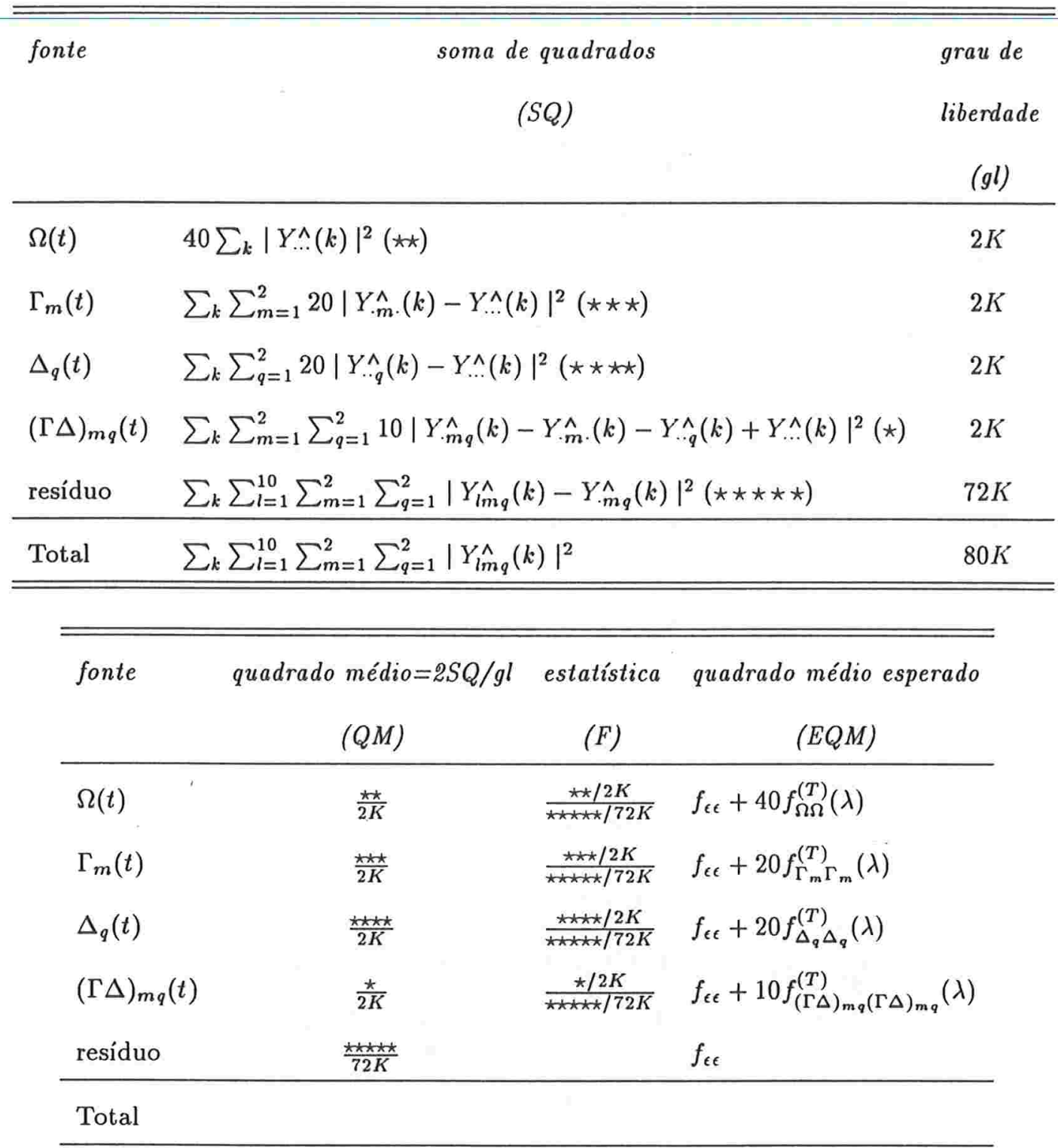

O teste para (6.1) pode ser realizado utilizando a estatística 


$$
F_{01}(\lambda)=\frac{\sum_{k} \sum_{m=1}^{2} \sum_{q=1}^{2} 10\left|Y_{\cdot m q}^{\wedge}(k)-Y_{\cdot m}^{\wedge} \cdot(k)-Y_{. q}^{\wedge}(k)+Y_{. . .}^{\wedge}(k)\right|^{2} / 2 K}{\sum_{k} \sum_{l=1}^{10} \sum_{m=1}^{2} \sum_{q=1}^{2}\left|Y_{l m q}^{\wedge}(k)-Y_{\cdot m q}^{\wedge}(k)\right|^{2} / 72 K}
$$

que, sob $H_{01}$, tem uma distribuição $\mathrm{F}(2 K, 72 K)$, para $0 \leq \lambda<\pi$.

A Figura 6.3 mostra os resultados da estatística (6.2), para $K=1$, que indica a existência, ao nível de 0,05 , de uma forte interação na frequência $\frac{2 \pi 57}{256}$ (período de 0,030 segundos). A existência de interação significa que o comportamento do eletroscilograma dos ratos numa determinada região depende do estado em que eles se encontram, ou seja, o comportamento do eletroscilograma dos ratos na região hipotálamo durante o sono paradoxal é diferente daquele durante o estado de alerta. As retas horizontais indicam os níveis críticos 0,05 e 0,01. Cada ponto da figura representa o grau de interação entre estado e região naquela frequência; quanto maior o valor da ordenada em uma determinada frequência mais forte será a interação. As frequências, onde a interação é significante, estão representadas na Tabela 6.2 .

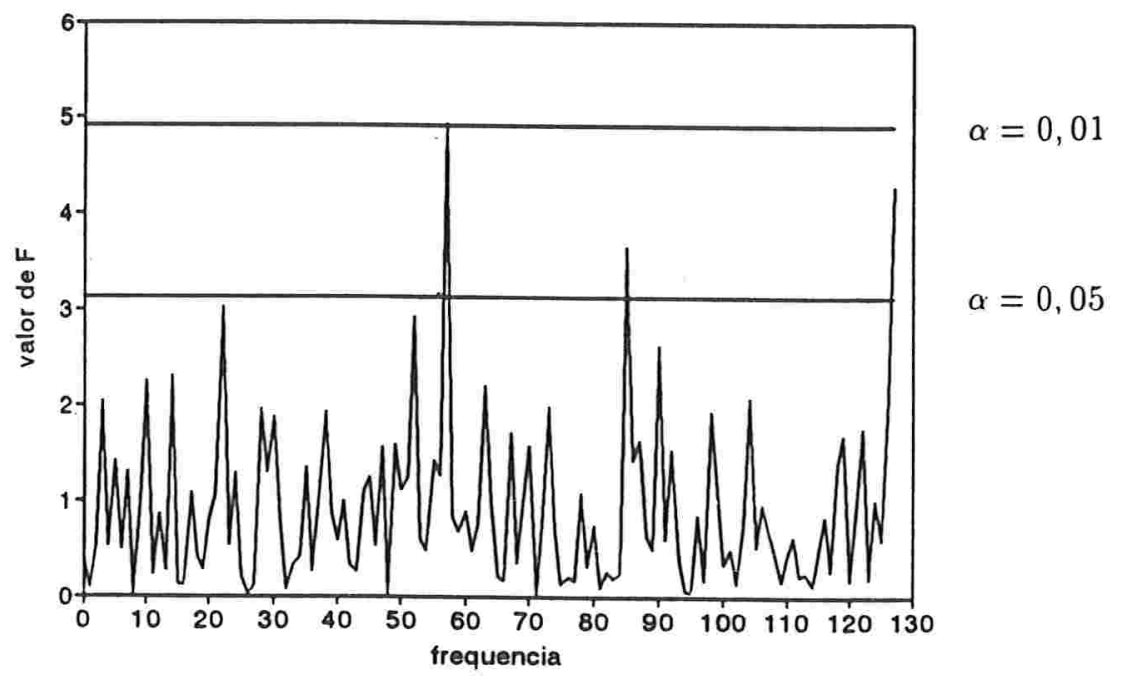

Figura 6.3. Estatística (6.2) para verificar a interação entre estado e região 
Tabela 6.2: Frequências selecionadas pelo teste (6.2)

\begin{tabular}{|c|c|c|c|c|}
\hline \hline índice & frequência & periodo & estatística $F$ & nível descritivo \\
\hline 57 & 1,399 & 0,030 & 4,9271 & 0,0099 \\
85 & 2,086 & 0,020 & 3,6567 & 0,0307 \\
127 & 3,117 & 0,013 & 4,2871 & 0,0174 \\
\hline \hline
\end{tabular}

Uma vez detectada a existência de interação no modelo, verificaremos, numa segunda etapa, se existe diferença entre os sinais do eletroscilograma na região do posteriotálamo e do hipotálamo durante o estado de alerta (comparação entre as regiões no estado de alerta) e, também, durante o sono paradoxal (comparação entre as regiões no estado de sono paradoxal). A análise de variância para comparação entre as regiões durante cada um dos estados está representada na Tabela 6.3.

Tabela 6.3: ANOVAF para comparação entre as regiões durante cada um dos estados

\begin{tabular}{lcc}
\hline \hline fonte & soma de quadrados & grau de liberdade \\
& $(S Q)$ & $(g l)$ \\
\hline$\Gamma_{m / 1}(t)$ & $\sum_{k} \sum_{m=1}^{2} 10\left|Y_{\cdot m 1}^{\wedge}(k)-Y_{. .1}^{\wedge}(k)\right|^{2}$ & $2 K$ \\
$\Gamma_{m / 2}(t)$ & $\sum_{k} \sum_{m=1}^{2} 10\left|Y_{\cdot m 2}^{\wedge}(k)-Y_{. .2}^{\wedge}(k)\right|^{2}$ & $2 K$ \\
resíduo & $\sum_{k} \sum_{l=1}^{10} \sum_{m=1}^{2} \sum_{q=1}^{2}\left|Y_{l m q}^{\wedge}(k)-Y_{\cdot m q}^{\wedge}(k)\right|^{2}$ & $72 K$ \\
\hline \hline
\end{tabular}

\begin{tabular}{|c|c|c|c|}
\hline fonte & $\begin{array}{c}\text { quadrado médio }=2 S Q / g l \\
(Q M)\end{array}$ & $\begin{array}{c}\text { estatística } \\
\text { (F) }\end{array}$ & $\begin{array}{c}\text { quadrado médio esperado } \\
\text { (EQM) }\end{array}$ \\
\hline$\Gamma_{m / 1}(t)$ & $\frac{\sum_{k} \sum_{m=1}^{2} \frac{10\left|Y \hat{m}_{1}(k)-Y . \hat{.}_{1}(k)\right|^{2}}{2 K}(\star)}{(k)}$ & $\frac{\hbar}{\star \star \star}$ & $f_{\epsilon \epsilon}+10 f_{\Gamma_{m 1} \Gamma_{m 1}}^{(T)}(\lambda)$ \\
\hline$\Gamma_{m / 2}(t)$ & $\frac{\sum_{k} \sum_{m=1}^{2} 10\left|Y_{m 2}^{\wedge}(k)-Y_{. .2}^{\wedge}(k)\right|^{2}}{2 K}(\star \star)$ & $\frac{\star \star \star}{\star \star \star}$ & $f_{\epsilon \epsilon}+10 f_{\Gamma_{m 2} \Gamma_{m 2}}^{(T)}(\lambda)$ \\
\hline resíduo & $\frac{\sum_{k} \sum_{l=1}^{10} \sum_{m=1}^{2} \sum_{q=1}^{2}\left|Y_{l m q}^{\wedge}(k)-Y_{\cdot_{q}}^{\wedge}(k)\right|^{2}}{72 K}(\star \star \star)$ & & $f_{\epsilon \epsilon}$ \\
\hline
\end{tabular}


onde

$\Gamma_{m / 1}(t)$ é o comportamento comum dos eletroscilogramas dos ratos na mésima região durante o estado de alerta, no instante t;

$\Gamma_{m / 2}(t)$ é o comportamento comum dos eletroscilogramas dos ratos na mésima região durante o estado de sono paradoxal, no instante t;

$f_{\Gamma_{m 1} \Gamma_{m 1}}^{(T)}(\lambda)=K^{-1} \sum_{k=1}^{K}\left|\Gamma_{m / 1}^{\wedge}(k)\right|^{2} ;$

$f_{\Gamma_{m 2} \Gamma_{m 2}}^{(T)}(\lambda)=K^{-1} \sum_{k=1}^{K}\left|\Gamma_{m / 2}^{\wedge}(k)\right|^{2}$.

No caso de comparação entre as regióes no estado de alerta, a hipótese a ser testada é

$$
H_{02}: \Gamma_{1 / 1}(t)=\Gamma_{2 / 1}(t)=0,
$$

e no caso de comparações entre as regiões durante sono paradoxal, a hipótese a ser testada é

$$
H_{03}: \Gamma_{1 / 2}(t)=\Gamma_{2 / 2}(t)=0 .
$$

O teste para (6.3) pode ser realizado utilizando a estatística

$$
F_{02}(\lambda)=\frac{\sum_{k} \sum_{m=1}^{2} 10\left|Y_{. m 1}^{\wedge}(k)-Y_{.: 1}^{\wedge}(k)\right|^{2} / 2 K}{\sum_{k} \sum_{l=1}^{10} \sum_{m=1}^{2} \sum_{q=1}^{2}\left|Y_{l m q}^{\wedge}(k)-Y_{\cdot m q}^{\wedge}(k)\right|^{2} / 72 K},
$$

que, sob $H_{02}$, tem uma distribuição $\mathrm{F}(2 K, 72 K)$, para $0 \leq \lambda<\pi$.

O teste para (6.4) também pode ser realizado utilizando a estatística

$$
F_{03}(\lambda)=\frac{\sum_{k} \sum_{m=1}^{2} 10\left|Y_{. m 2}^{\wedge}(k)-Y_{. .2}^{\wedge}(k)\right|^{2} / 2 K}{\sum_{k} \sum_{l=1}^{10} \sum_{m=1}^{2} \sum_{q=1}^{2}\left|Y_{l m q}^{\wedge}(k)-Y_{\cdot m}^{\wedge}(k)\right|^{2} / 72 K},
$$

que, sob $H_{03}$, tem uma distribuição $\mathrm{F}(2 K, 72 K)$, para $0 \leq \lambda<\pi$.

Os resultados, para $K=1$, estão apresentados na Figura 6.4 (comparação entre as regiões no estado de alerta) e Figura 6.5 (comparação entre as regiões durante o estado de sono paradoxal). As linhas horizontais indicam os níveis críticos 0,05 e 0,025 de uma distribuição $F(2,72)$. Cada um dos pontos das Figuras 6.4 e 6.5 representa o grau de diferença entre os periodogramas dos ratos na região do hipotálamo e do posteriotálamo, durante cada 
um dos estados, isto é, quanto maior o valor da ordenada em uma determinada frequência maior será a diferença dos periodogramas entre os ratos de diferentes regiões, durante cada um dos estados de sono. Os valores das estatísticas (6.5) e (6.6) nas frequências significantes estão nas Tabelas 6.4 e 6.5 .

Analisando os resultados da Tabela 6.4 vemos que existe uma diferença, entre as regiões do cérebro, no comportamento dos eletroscilogramas nas frequências $\frac{2 \pi 17}{256}$ (correspondente a um período de 0,1 segundos), $\frac{2 \pi 99}{256}$ (período de 0,017 segundos) e $\frac{2 \pi 104}{256}$ (período de 0,016 segundos), durante o estado de alerta.

Durante o estado de sono paradoxal, Tabela 6.5, também existe uma diferença, entre as regiões do cérebro, no comportamento dos eletroscilogramas. Entretanto, essa diferença se manifesta de forma diferente do caso anterior, isto é, em um número maior e diferente de frequências.

Estes resultados são consistentes com a existência de interação entre estado e região.

Numa terceira etapa podemos construir os eletroscilogramas comuns para os ratos de cada um dos grupos: PAR, PSR, HAR e HSR.

Para esta análise utilizamos o modelo (4.9)

$$
Y_{n}(t)=\mu_{n}+\Omega(t)+\epsilon_{n}(t), \quad n=1, \cdots, 10,
$$

e testamos a hipótese

$$
H_{04}: \Omega(t)=0 \text {, }
$$

através da estatística (4.18), para cada um dos grupos. 


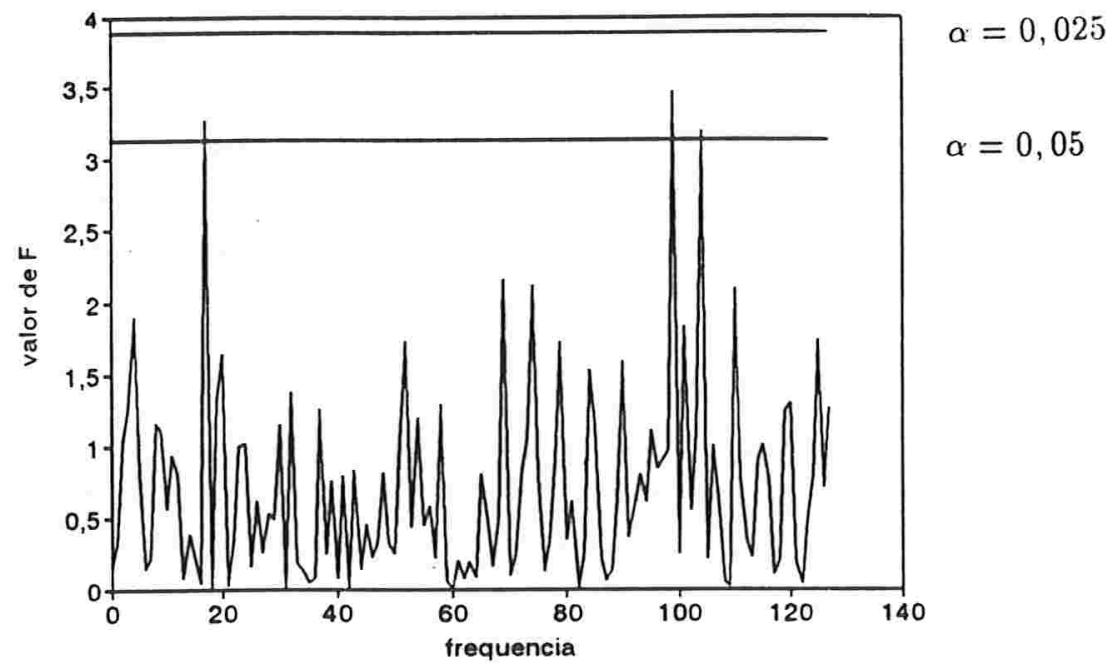

Figura 6.4. Estatística (6.5) para comparação de sinais entre as regiões durante o estado de alerta

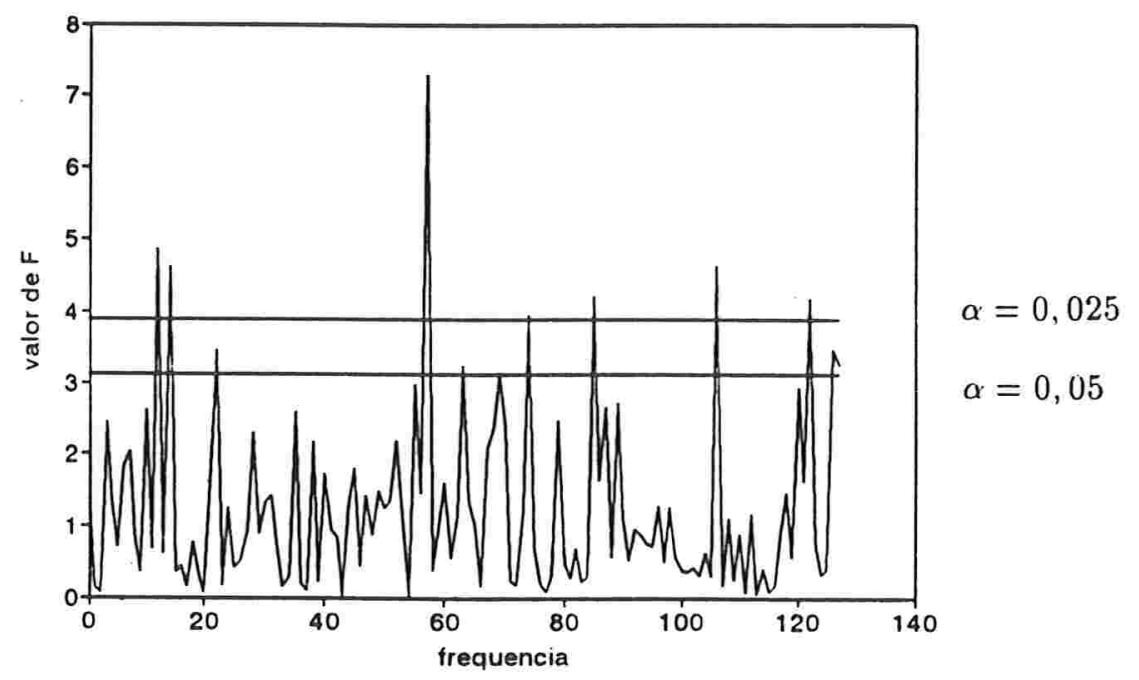

Figura 6.5. Estatística (6.6) para comparação de sinais entre as regiões durante o estado de sono paradoxal 
Tabela 6.4: Frequências selecionadas pelo teste (6.5)

(comparação entre as regiões do cérebro durante o estado de alerta)

\begin{tabular}{|c|c|c|c|c|}
\hline \hline índice & frequência & periodo & estatística $F$ & nível descritivo \\
\hline 17 & 0,417 & 0,100 & 3,2668 & 0,0438 \\
99 & 2,430 & 0,017 & 3,4666 & 0,0365 \\
104 & 2,553 & 0,016 & 3,1872 & 0,0472 \\
\hline \hline
\end{tabular}

Tabela 6.5: Frequências selecionadas pelo teste (6.6)

(comparação entre as regiões do cérebro durante o estado de sona paradoxal)

\begin{tabular}{|c|c|c|c|c|}
\hline \hline indice & frequência & periodo & estatística $F$ & nível descritivo \\
\hline 12 & 0,294 & 0,142 & 4,8712 & 0,0104 \\
14 & 0,344 & 0,121 & 4,6262 & 0,0129 \\
22 & 0,540 & 0,077 & 3,4545 & 0,0369 \\
57 & 1,399 & 0,030 & 7,2849 & 0,0013 \\
63 & 1,546 & 0,027 & 3,2488 & 0,0449 \\
69 & 1,693 & 0,025 & 3,1496 & 0,0446 \\
74 & 1,816 & 0,023 & 3,9623 & 0,0233 \\
85 & 2,086 & 0,020 & 4,2199 & 0,0185 \\
106 & 2,601 & 0,016 & 4,6447 & 0,0127 \\
122 & 2,994 & 0,014 & 4,1909 & 0,0190 \\
126 & 3,093 & 0,013 & 3,4759 & 0,0362 \\
127 & 3,117 & 0,013 & 3,2475 & 0,0446 \\
\hline \hline
\end{tabular}


Os resultados estão na Figura 6.6, onde cada ponto representa o grau de similaridade entre os periodogramas dos ratos naquela frequência, isto é, para cada grupo, quanto maior o valor da ordenada em uma determinada frequência mais parecidos serão os periodogramas dos ratos naquela frequência. As retas horizontais representam os níveis críticos $0,05,0,025$, 0,01 e 0,005 de uma distribuição $F(2,18)$. Os valores das ordenadas superiores a essas retas corresponderão às frequências comuns presentes nos eletroscilogramas dos ratos do mesmo grupo. Notamos que, ao nível de 0,05 , temos oito frequências comuns nos grupos PAR e HAR, sete nos grupos PSR e HSR; ao nível de 0,025, temos quatro frequências comuns nos grupos PAR, HAR e PSR, e uma no grupo HSR; ao nível de 0,01 , temos três nos grupos PAR e HAR, duas no grupo PSR e nenhuma no grupo HSR enquanto ao nível de 0,005, uma no grupo PAR, duas no grupo HAR e nenhuma nos grupos PSR e HSR.

As Tabelas 6.6 a 6.9 apresentam as frequências comuns, cujos níveis críticos são inferiores a 0,05 , para cada um dos grupos. No grupo PAR, o maior pico é $F(110)=8,8296$, significando que o componente correspondente a um período de 0,016 segundos é um forte componente comum. Analogamente, no grupo HAR é $F(52)=9,0022$ que corresponde a um período de 0,033 segundos, no grupo PSR é $F(67)=6,814$ correspondente a um período de 0,025 segundos, e no grupo HSR é $F(110)=4,615$ correspondente a um período de 0,016 segundos. Notamos também que os grupos PAR e HSR têm um forte componente comum em alta frequência (período curto), enquanto os grupos HAR e PSR têm um forte componente comum em baixa frequência (período longo).

Com base nas Tabelas 6.6 a 6.9 construímos a "série comum" de cada um dos grupos, isto é, a série que só contem as frequências comuns em cada um deles. Os resultados estão na Figura 6.7. Os periodogramas correspondentes a essas séries comuns estão na Figura 6.8. De uma forma geral, podemos notar que os maiores valores dos periodogramas estão localizados em baixas frequências, estatisticamente isto significa que as baixas frequências explicam a maior parte da variabilidade dos sinais comuns obtidos. 

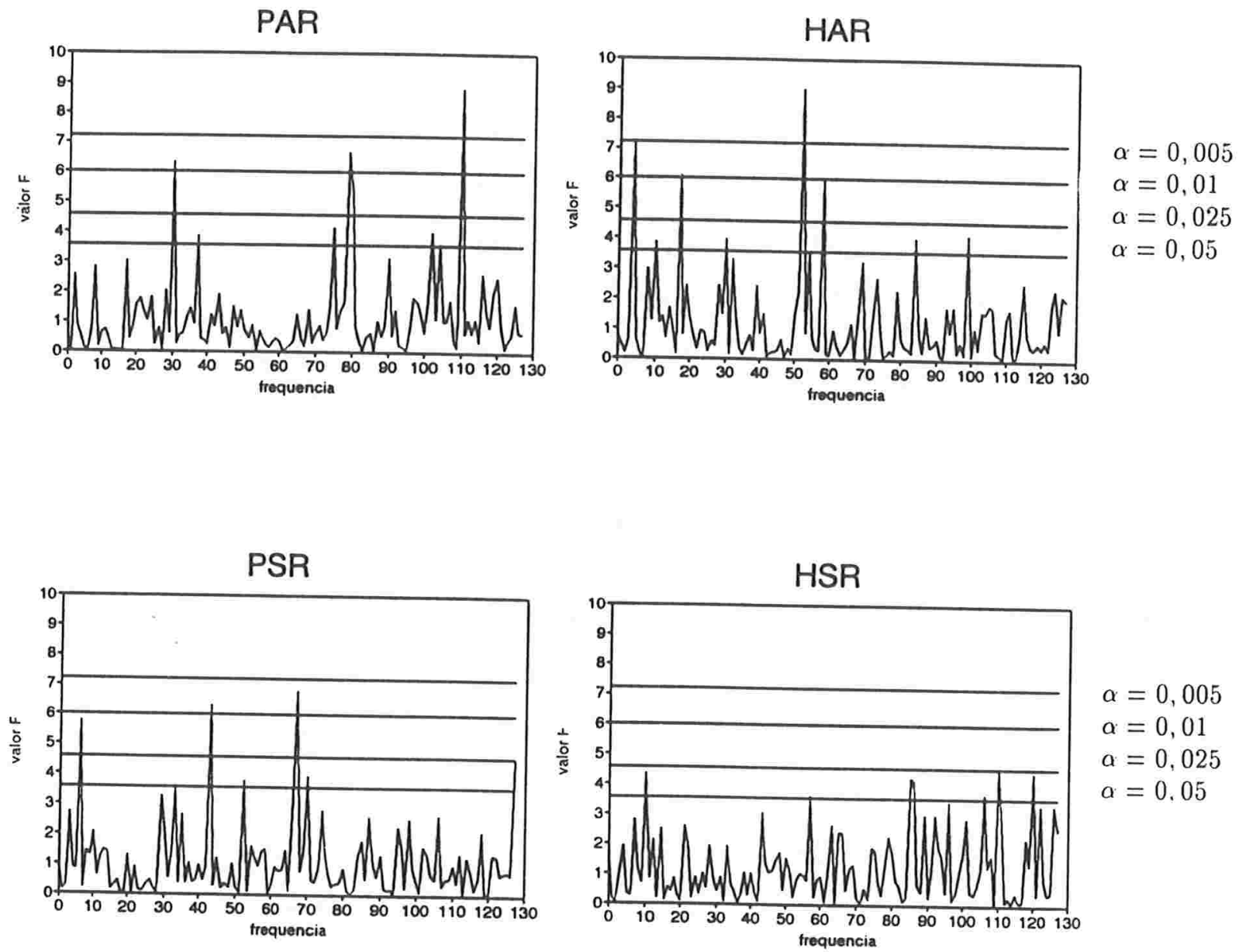

Figura 6.6. Estatística (4.18) para testar um sinal comum em cada um dos grupos 
Tabela 6.6: Frequências comuns no grupo PAR

\begin{tabular}{|c|c|c|c|c|}
\hline \hline índice & frequência & período & estatística $F$ & nível descritivo \\
\hline 30 & 0,736 & 0,057 & 6,32006 & 0,0083 \\
37 & 0,908 & 0,046 & 3,87967 & 0,0397 \\
75 & 1,841 & 0,023 & 4,14205 & 0,0331 \\
79 & 1,939 & 0,022 & 6,67442 & 0,0068 \\
80 & 1,963 & 0,021 & 5,32180 & 0,0153 \\
102 & 2,503 & 0,017 & 3,98325 & 0,0370 \\
104 & 2,552 & 0,016 & 3,60251 & 0,0483 \\
110 & 2,699 & 0,015 & 8,82965 & 0,0021 \\
\hline \hline
\end{tabular}

Tabela 6.7: Frequências comuns no grupo HAR

\begin{tabular}{|c|c|c|c|c|}
\hline \hline indice & frequência & período & estatística $F$ & nível descritivo \\
\hline 4 & 0,098 & 0,427 & 7,24691 & 0,0049 \\
10 & 0,245 & 0,171 & 3,87470 & 0,0399 \\
17 & 0,417 & 0,100 & 6,09668 & 0,0095 \\
30 & 0,736 & 0,057 & 3,96384 & 0,0375 \\
52 & 1,276 & 0,056 & 9,00219 & 0,0020 \\
58 & 1,423 & 0,029 & 5,99777 & 0,0101 \\
84 & 2,061 & 0,020 & 4,04116 & 0,0355 \\
99 & 2,429 & 0,017 & 4,14768 & 0,0330 \\
\hline \hline
\end{tabular}


Tabela 6.8: Frequências comuns no grupo PSR

\begin{tabular}{|c|c|c|c|c|}
\hline \hline índice & frequência & período & estatística $F$ & nível descritivo \\
\hline 6 & 0,147 & 0,283 & 5,77492 & 0,0115 \\
33 & 0,810 & 0,052 & 3,60931 & 0,0481 \\
43 & 1,055 & 0,040 & 6,32910 & 0,0083 \\
52 & 1,276 & 0,033 & 3,79633 & 0,0421 \\
67 & 1,644 & 0,025 & 6,81400 & 0,0063 \\
70 & 1,718 & 0,024 & 3,95908 & 0,0376 \\
127 & 3,117 & 0,013 & 4,58574 & 0,0246 \\
\hline \hline
\end{tabular}

Tabela 6.9: Frequências comuns no grupo HSR

\begin{tabular}{|c|c|c|c|c|}
\hline \hline índice & frequência & período & estatística $F$ & nível descritivo \\
\hline 10 & 0,245 & 0,170 & 4,37215 & 0,0283 \\
57 & 1,399 & 0,030 & 3,64982 & 0,0467 \\
85 & 2,086 & 0,020 & 4,25211 & 0,0307 \\
86 & 2,110 & 0,020 & 4,16119 & 0,0327 \\
106 & 2,601 & 0,016 & 3,70882 & 0,0448 \\
110 & 2,699 & 0,015 & 4,61495 & 0,0241 \\
120 & 2,945 & 0,014 & 4,44101 & 0,0271 \\
\hline \hline
\end{tabular}



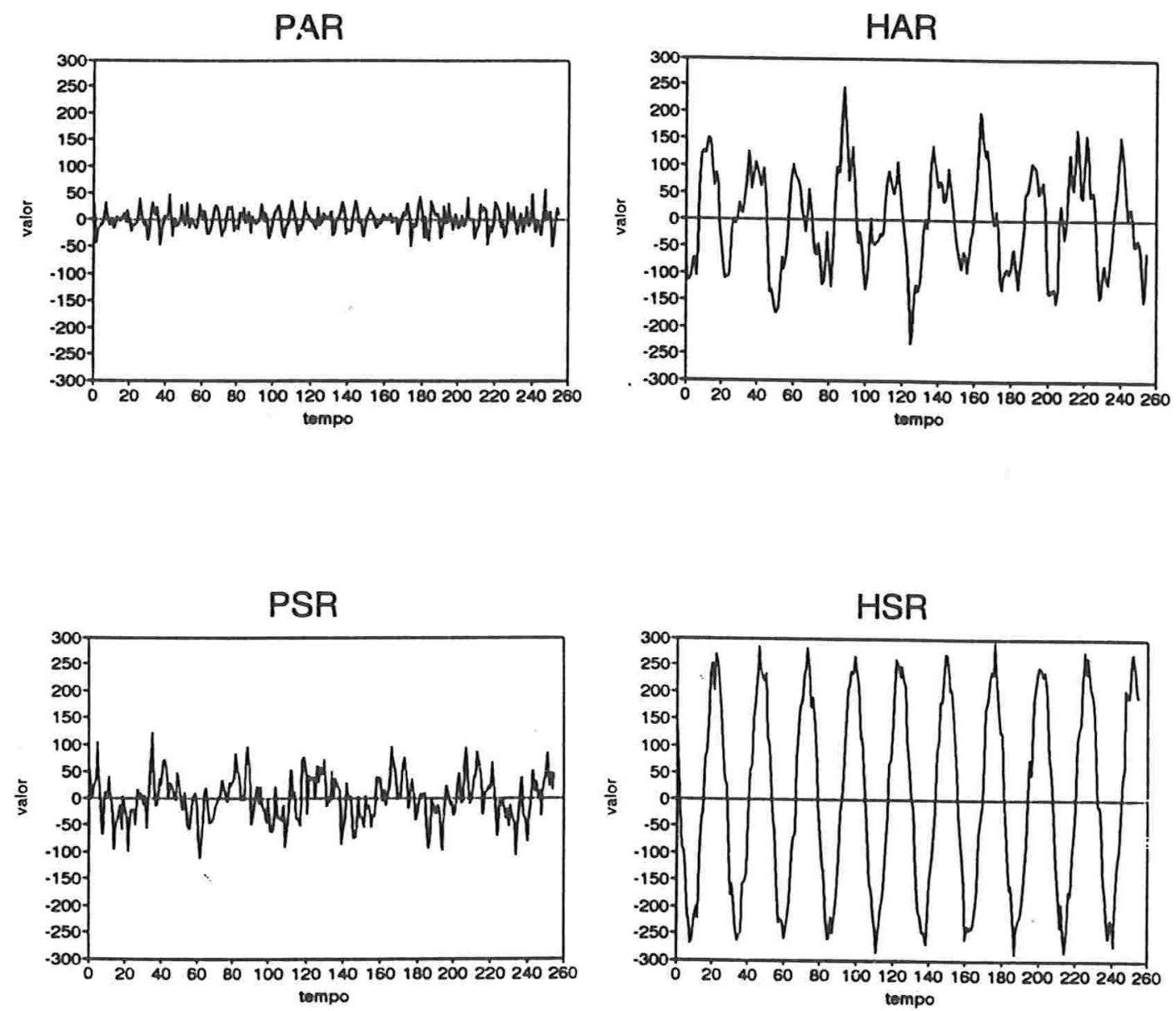

Figura 6.7. Série comum para cada um dos grupos ao nível de 0,05 

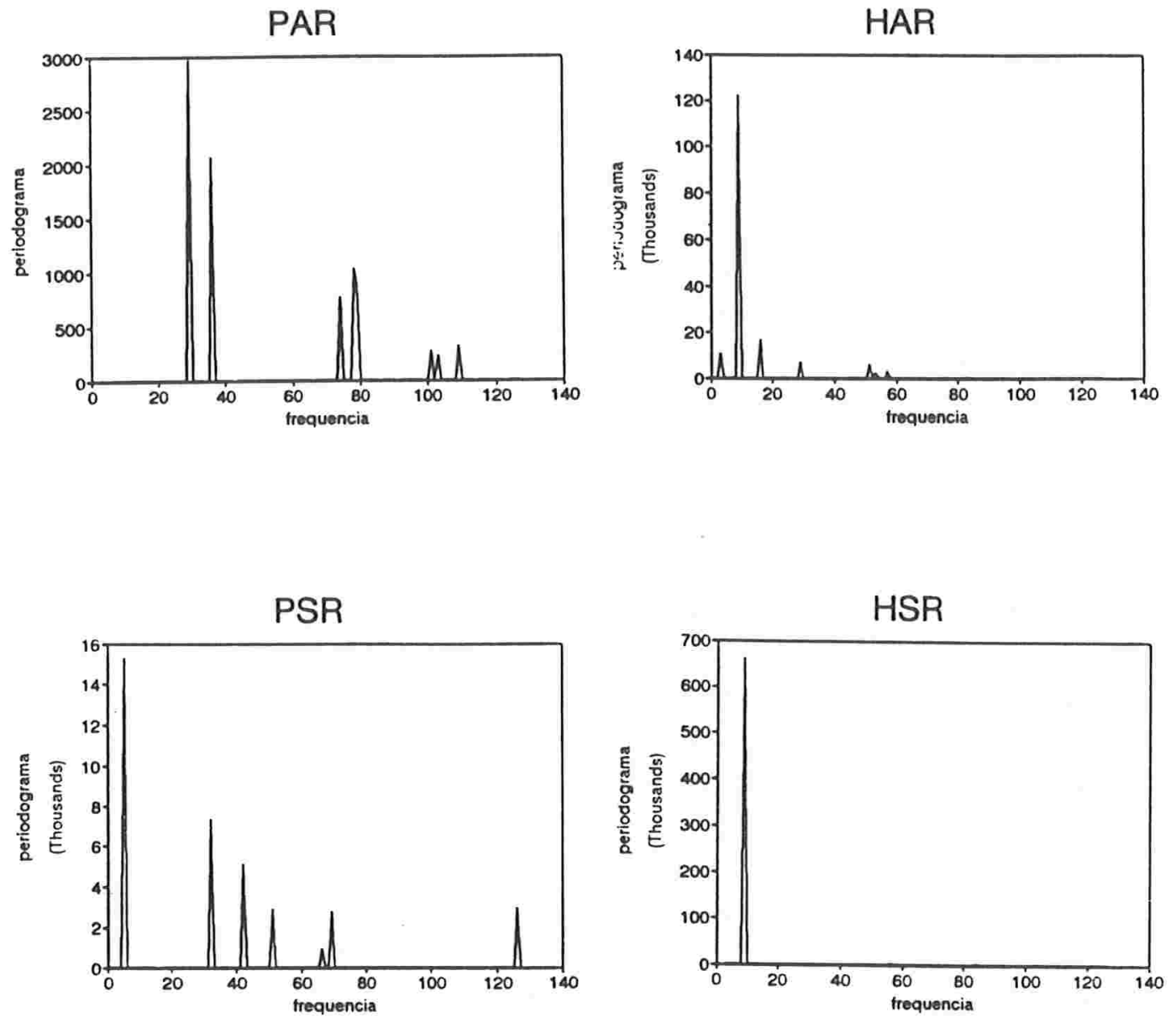

Figura 6.8. Periodograma da série comum para cada um dos grupos 


\subsection{Análise de Variância usando a transfor- mada de Walsh-Fourier}

Foram selecionados dados de Cíclo Vigília/Sono de oito indivíduos da cidade de Natal (Rio Grande do Norte)(RN) e oito indivíduos da cidade de Pelotas (Rio Grande do Sul)(RS), a partir de uma amostra de 78 pessoas, adultas, de 20 a 40 anos de idade, de ambos os sexos, residentes em regióes urbanas. Esta coleta de dados fez parte do projeto desenvolvido pelo Grupo Multidisciplinar de Desenvolvimento e Rítmos Biológicos do ICB/USP que tem por objetivo analisar os efeitos sobre os rítmos biológicos da instalação e retirada do horário de verão.

Os sujeitos anotaram voluntariamente seus horários de início e fim de sono, início e fim de sestas. Para efeito deste trabalho foram considerados apenas os primeiros 23 dias (552 pontos) que antecederam a instalação do horário de verão, não contendo estes dados, portanto, as possíveis alterações produzidas pelo adiantamento de uma hora nos horários sociais. Para garantir a equidistância dos dados, as durações de todos os episódios de sono, incluíndo as sestas, foram divididas em intervalos de uma hora. $\mathrm{O}$ critério de construção de cada série temporal, sendo o sono a variável, foi o seguinte: se o sujeito estivesse dormindo foi atribuído o valor 1 , e se estivesse acordado foi atribuído o valor 0 .

O objetivo é a aplicação da Transformada de Walsh às séries temporais correspondentes ao Cíclo Vigília/Sono dos dois grupos (RN e RS) de indivíduos, construir os periodogramas de Walsh e compará-los. A diferença entre eles aponta para eventuais diferenças nos hábitos de sono, em função das diferentes latitudes das cidades de Natal e Pelotas e suas possíveis consequências.

Inicialmente, utilizamos 512 pontos de cada série para que o tamanho da série seja uma potência de 2 e possamos aplicar o algoritmo FWT (transformada rápida de Walsh) para fazer as análises, ver Ahmed e Rao(1975). Os dados estão apresentados nas Figura 6.9 e 6.10, sendo que os primeiros oito indivíduos (de 1 a 8 ) são da cidade de $\mathrm{Natal}(\mathrm{RN})$ e os restantes (de 9 a 16) são da cidade de Pelotas (RS). Notamos uma similaridade no comportamento 
dos indivíduos, porém houve um maior número de mudanças do estado de sono para o grupo RN.

As Figuras 6.11 e 6.12 mostram os periodogramas de Walsh, onde verificase a existência de um forte pico na sequência $\frac{2 \pi 42}{512}$ (índice de 42 ) correspondente a um periodo de 12 horas em todas as séries, isto é, existe uma mudança de estado a cada 12 horas. Além disso, notamos também que as sequências baixas apresentam a maior parte da variabilidade dos estados de sono de cada indivíduo.

A Figura 6.13 mostra o periodograma médio de cada grupo. Notamos que os dois periodogramas são similares, o maior pico está na sequência $\frac{2 \pi 42}{512}$ (índice de 42) correspondente a um período de 12 horas para cada grupo.

Consideremos, inicialmente, o problema de detectar a existência de um sinal comum em cada um dos grupos. Para esta análise, utilizamos o modelo $(5.6)$

$$
X_{n}(t)=\Theta_{n}+\Omega(t)+\epsilon_{n}(t)
$$

onde

$X_{n}(t)$ é o estado de sono do n-ésimo indivíduo, no instante $t$;

$\Theta_{n}$ é um valor médio dos estados de sono do n-ésimo indivíduo;

$\Omega(t)$ é um comportamento comum de sono de todos os indivíduos, no instante $\mathrm{t}$;

$\epsilon_{n}(t)$ é o erro aleatório

e testamos a hipótese

$$
H_{05}: \Omega(t)=0
$$

através da estatística (5.14).

Os resultados do grupo RN estão apresentados na Figura 6.14; e os do grupo RS na Figura 6.15. As linhas horizontais indicam os níveis críticos $0,05,0,01$ e 0,001 de uma distribuição $F(1,7)$. Cada ponto das figuras representa o grau de similaridade, naquela sequência, dos periodogramas de Walsh dos indivíduos do grupo. 

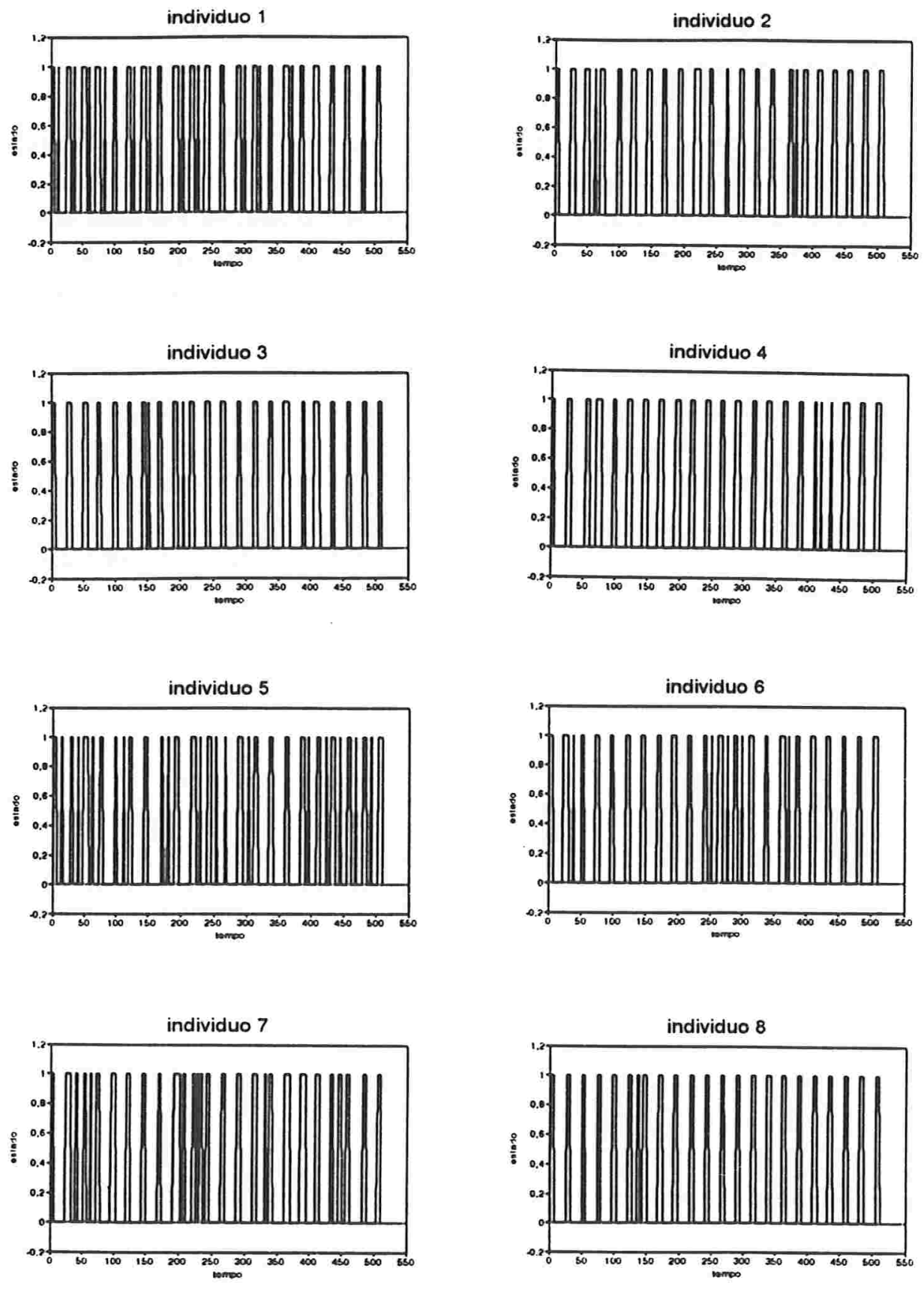

Figura 6.9. Estado de sono de cada indivíduo do Rio Grande do Norte 

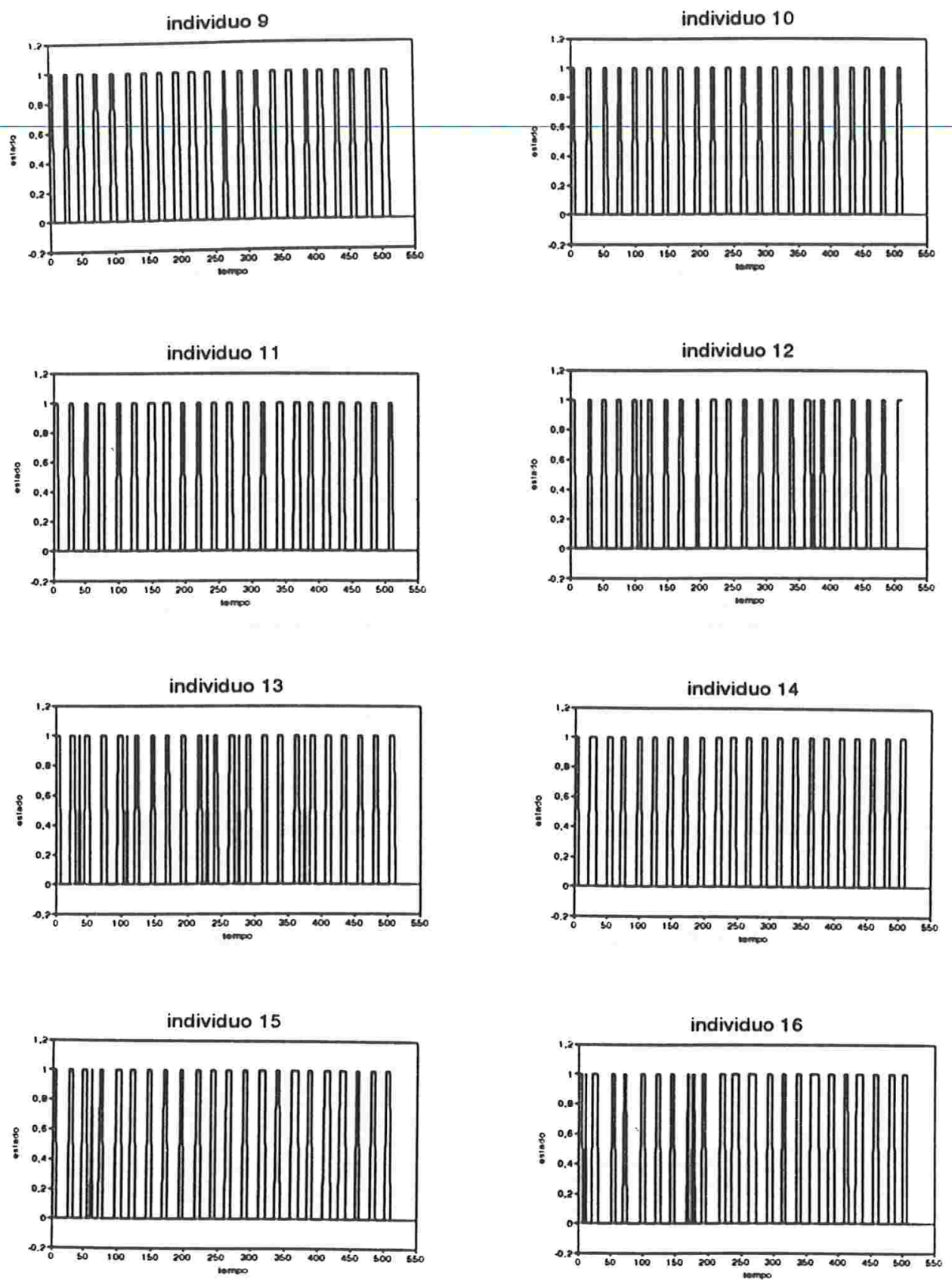

Figura 6.10. Estado de sono de cada indivíduo do Rio Grande do Sul 

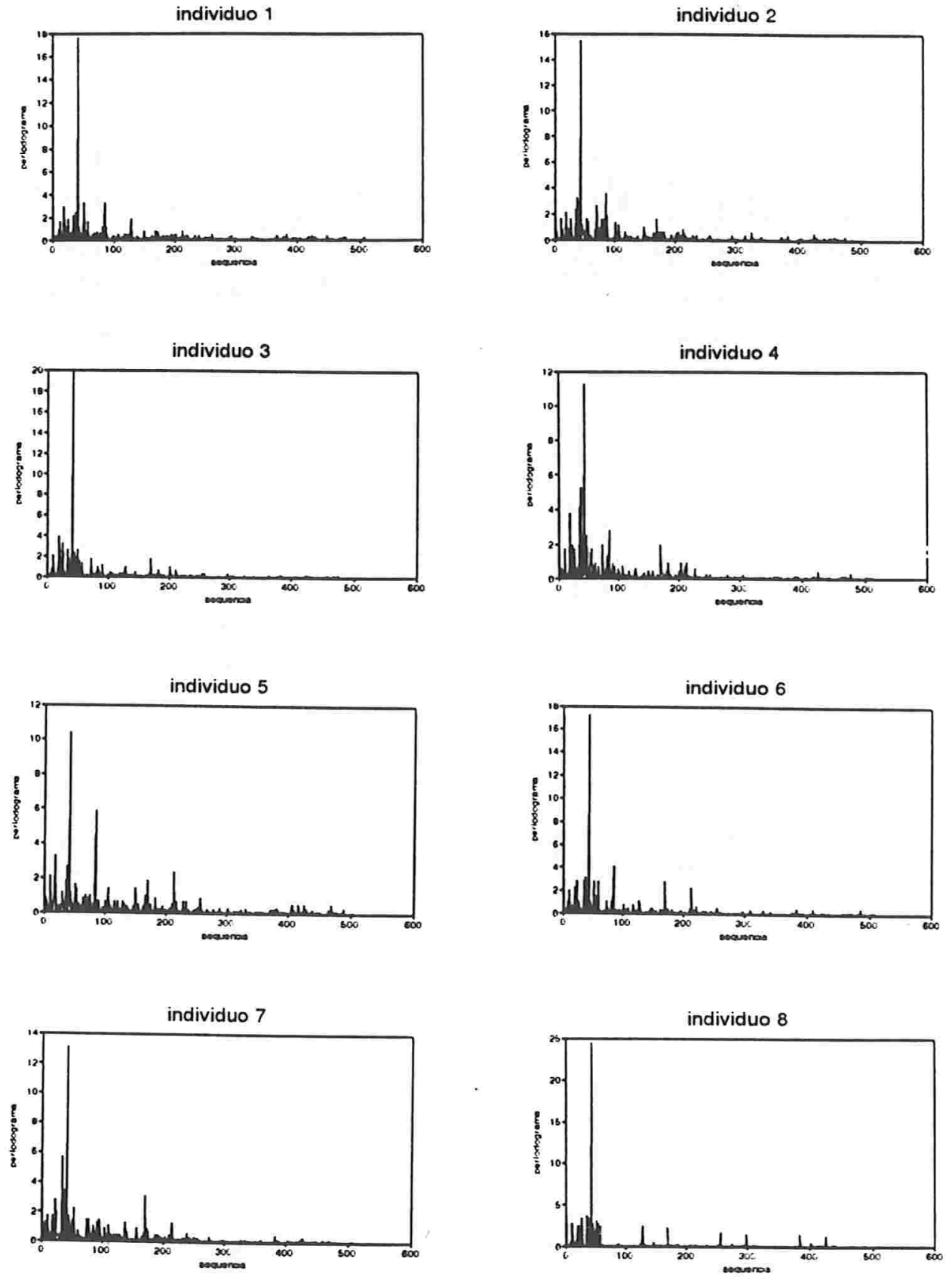

Figura 6.11. Periodograma de Walsh de cada indivíduo do Rio Grande do Norte 

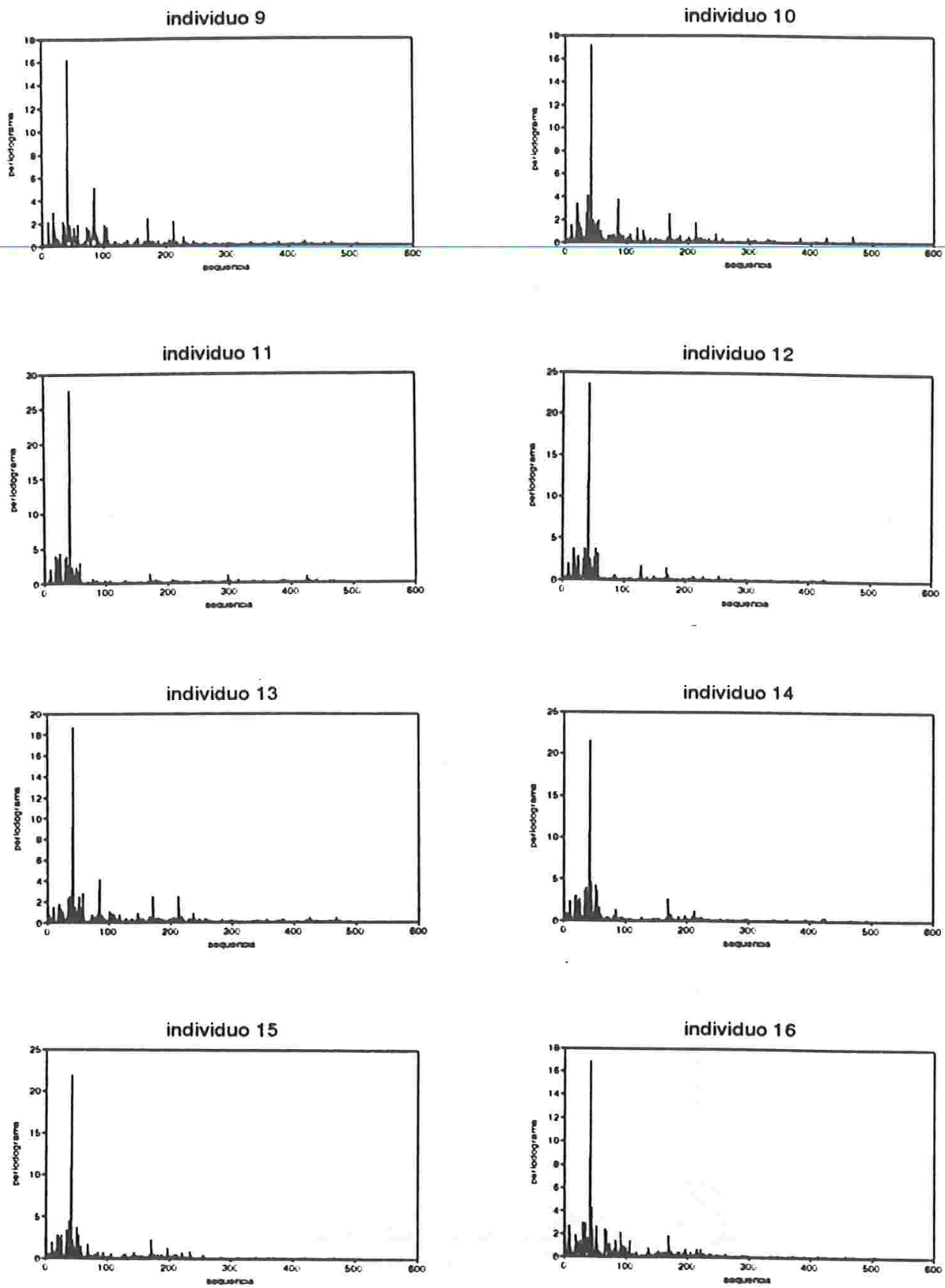

Figura 6.12. Periodograma de Walsh de cada indivíduo do Rio Grande do $\mathrm{Sul}$ 
RN

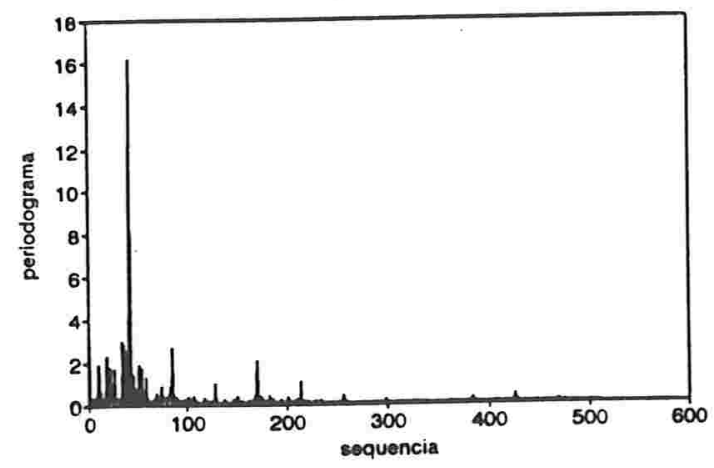

RS

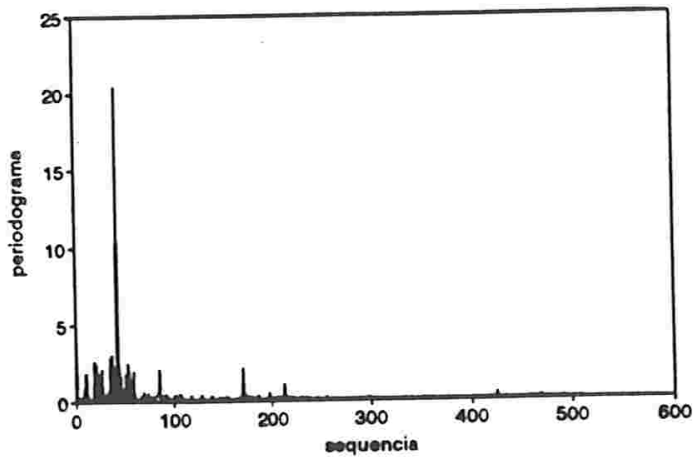

Figura 6.13. Periodograma médio de Walsh para os grupos RN e RS

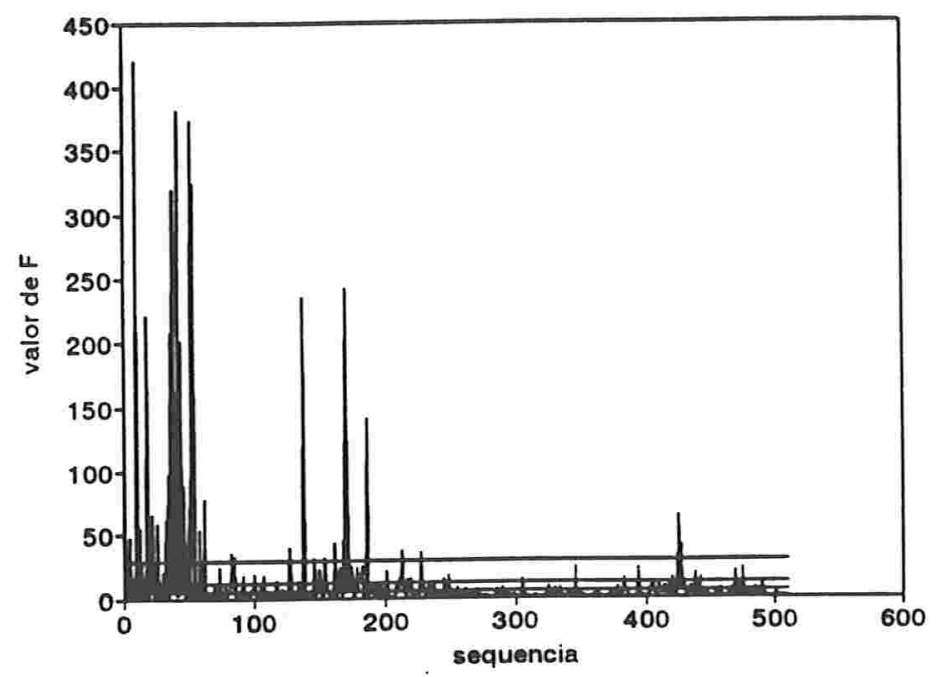

Figura 6.14. Estatística (5.14) para detectar um sinal comum do grupo RN 


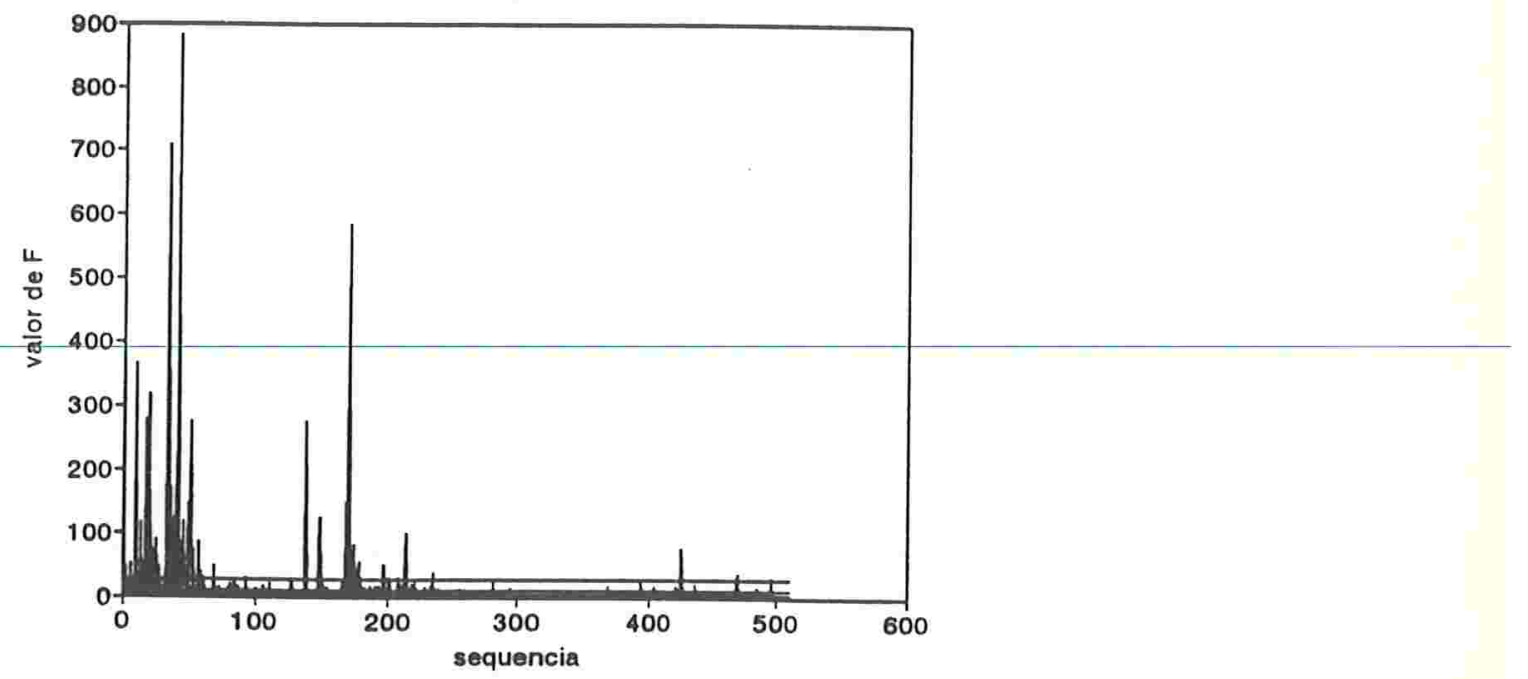

Figura 6.15. Estatística (5.14) para detectar um sinal comum do grupo RS

Analisando as duas últimas figuras, podemos concluir ao nível de 0,05 , que existem sinais comuns em cada um dos dois grupos (RN e RS) e, também, que a maioria deles estão concentrados em sequências baixas.

A Figura 6.16 representa a "série comum" de cada um dos grupos, cuja construção foi baseada nas Figuras 6.14 e 6.15; cada uma delas só contem as sequências comuns do correspondente grupo. Os periodogramas correspondentes a essas séries comuns estão na Figura 6.17. De uma forma geral, podemos notar que os maiores valores dos periodogramas estão localizados em baixas sequências.

Após a conclusão da existência de sinais comuns em cada grupo, podemos estender a análise para verificar se existe diferença entre os sinais comuns de cada um dos grupos. Para isso, utilizamos o modelo (5.15),

$$
X_{l m}(t)=\Theta_{l m}+\Omega_{m}(t)+\epsilon_{l m}(t),
$$

onde

$X_{l m}(t)$ é o estado de sono do l-ésimo indivíduo do m-ésimo grupo ( $\mathrm{RN}$ ou RS), no instante $t, l=1, \cdots, 8$ e $m=1,2$;

$\Theta_{l m}$ é um valor médio dos estados de sono do l-ésimo indivíduo do m-ésimo grupo; 
$\Omega_{m}(t)$ é o comportamento comum de sono do m-ésimo grupo, no instante $t$;

$\epsilon_{l m}(t)$ é o erro aleatório

e testamos a hipótese

$$
H_{06}: \Omega_{1}(t)=\Omega_{2}(t)=0,
$$

através da estatística (5.28).

A Figura 6.18 apresenta os resultados e indica os níveis críticos $0,05,0,01$ e 0,001 de uma distribuição $F(1,14)$. Cada ponto da figura representa o grau de diferença entre os indivíduo do grupo RN e do grupo RS. As sequências selecionadas pela estatística (5.28) estão listadas na Tabela 6.10 , onde notamos que existe uma diferença muito grande dos sinais entre os grupos nos períodos de 1,15 e 1,08 horas (índice de 445 e 476, respectivamente); esses sinais são essencialmente em períodos curtos (nas sequências altas). Também notamos que não existem diferenças de sinais em baixas sequências, ou seja, em períodos longos.
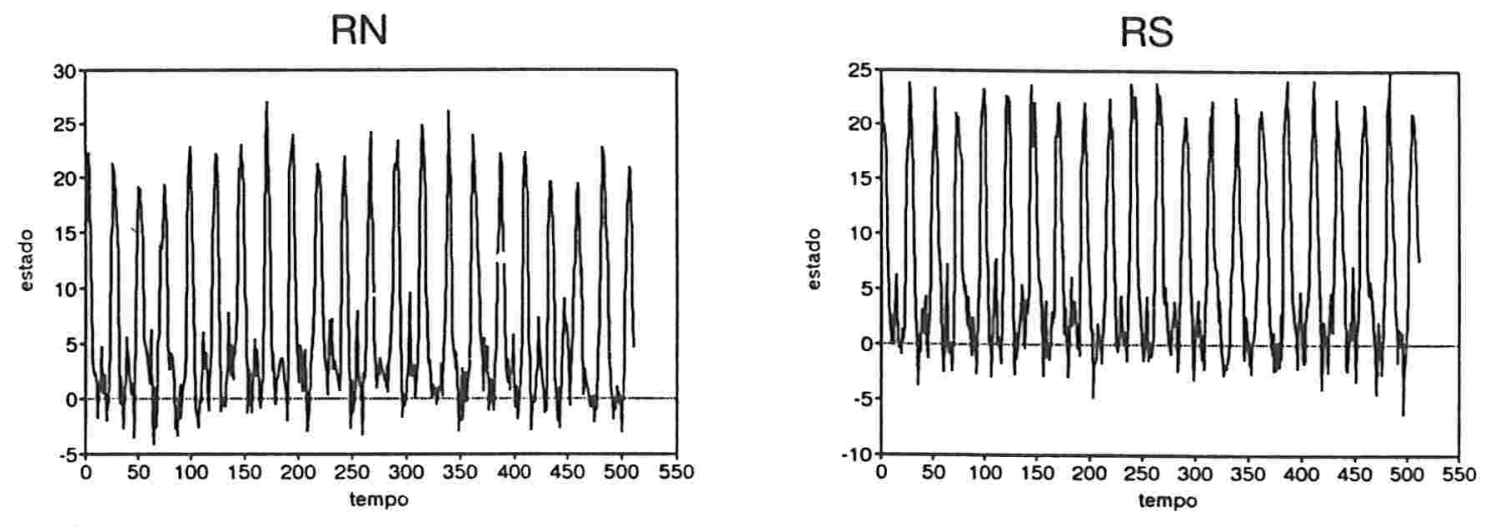

Figura 6.16. Série comum para cada um dos grupos ao nível de 0,01 

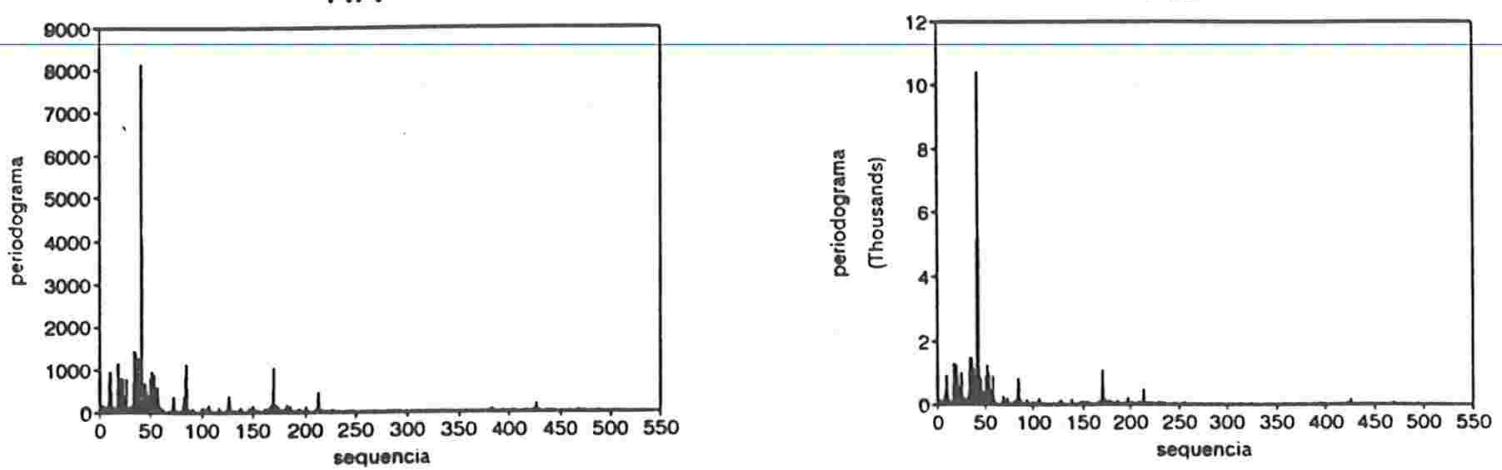

Figura 6.17. Periodograma da série comum para cada um dos grupos

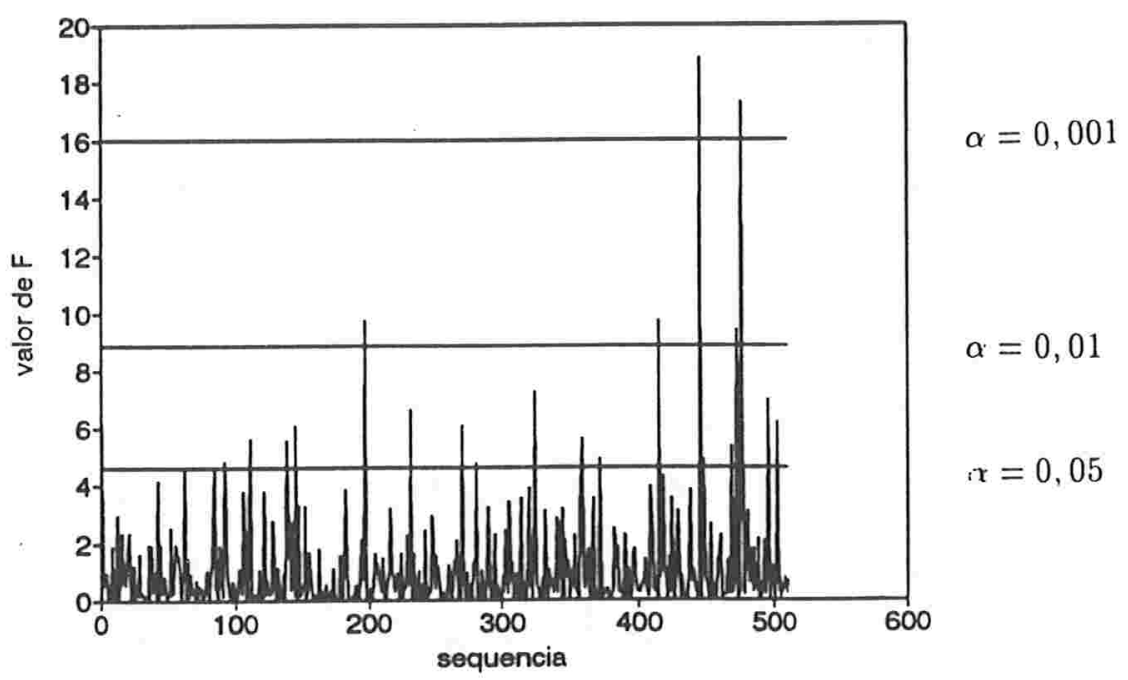

Figura 6.18. Estatística (5.28) para comparaçào dos sinais entre os grupos RN e RS 
Tabela 6.10: Sequências selecionadas pelo teste (5.28) comparação de sinais entre os grupos RN e RS

\begin{tabular}{|c|c|c|c|c|}
\hline \hline índice & sequência & periodo & estatística $F$ & nível descritivo \\
\hline 1 & 0,002 & 512,00 & 6,1394 & 0,0266 \\
92 & 0,180 & 5,57 & 4,8172 & 0,0455 \\
111 & 0,217 & 4,61 & 5,6435 & 0,0323 \\
138 & 0,270 & 3,71 & 5,5556 & 0,0335 \\
145 & 0,283 & 3,53 & 6,0892 & 0,0271 \\
197 & 0,385 & 2,60 & 9,7554 & 0,0075 \\
232 & 0,453 & 2,21 & 6,6602 & 0,0218 \\
270 & 0,527 & 1,90 & 6,0952 & 0,0270 \\
281 & 0,549 & 1,82 & 4,7914 & 0,0460 \\
325 & 0,635 & 1,58 & 7,2846 & 0,0173 \\
360 & 0,703 & 1,42 & 5,6435 & 0,0323 \\
373 & 0,729 & 1,37 & 4,9296 & 0,0434 \\
415 & 0,811 & 1,23 & 9,7704 & 0,0074 \\
445 & 0,869 & 1,15 & 18,8222 & 0,0007 \\
448 & 0,875 & 1,14 & 4,8959 & 0,0440 \\
468 & 0,914 & 1,09 & 5,3567 & 0,0363 \\
472 & 0,922 & 1,08 & 9,4595 & 0,0082 \\
475 & 0,928 & 1,08 & 5,1882 & 0,0390 \\
476 & 0,930 & 1,07 & 17,3077 & 0,0009 \\
496 & 0,969 & 1,03 & 7,0000 & 0,0192 \\
503 & 0,982 & 1,02 & 6,1967 & 0,0260 \\
\hline \hline
\end{tabular}




\subsection{Comparação entre a análise de Walsh- Fourier e a de Fourier}

Reanalisando os dados de Cíclo Vigília/Sono, agora utilizando a transformada de Fourier, obtemos os seguintes resultados.

O periodograma médio de Fourier de cada grupo (RN e RS) é apresentado na Figura 6.19. Para fazer as comparações entre a análise de Walsh-Fourier e a de Fourier, o periodograma médio de Walsh de cada grupo (Figura 6.13) é modificado utilizando a "H-sequency", cujo resultado está apresentado na Figura 6.20. Comparando as Figuras 6.19 e 6.20, podemos notar que os dois periodogramas de cada grupo são muito similares, e ambos apresentam um forte pico em torno de $\lambda=\frac{21}{512}$. No domínio de frequência, $\lambda$ é medido em ciclos por hora, isto é, existe um ciclo a cada 24 horas ( $\frac{512}{21}$ horas). No domínio de "H-sequency", a quantidade 24 horas é interpretada como sendo o período médio das oscilações dominantes, isto é, o intervalo médio de mudança de estado multiplicado por 2 .

As diferenças mais notáveis entre os dois periodogramas de cada grupo são os picos que aparecem no periodograma de Walsh em "H-sequency" baixas e não aparecem no periodograma de Fourier. Estas "H-sequency" correspondem a parte da variabilidade dos estados de sono que são perdidas na análise de Fourier.

Estes resultados são consistentes, uma vez que os dados analisados têm um comportamento mais proximo de "ondas quadradas" do que de senóides (Figuras 6.9 e 6.10); neste caso a análise de Walsh-Fourier é mais adequada. 

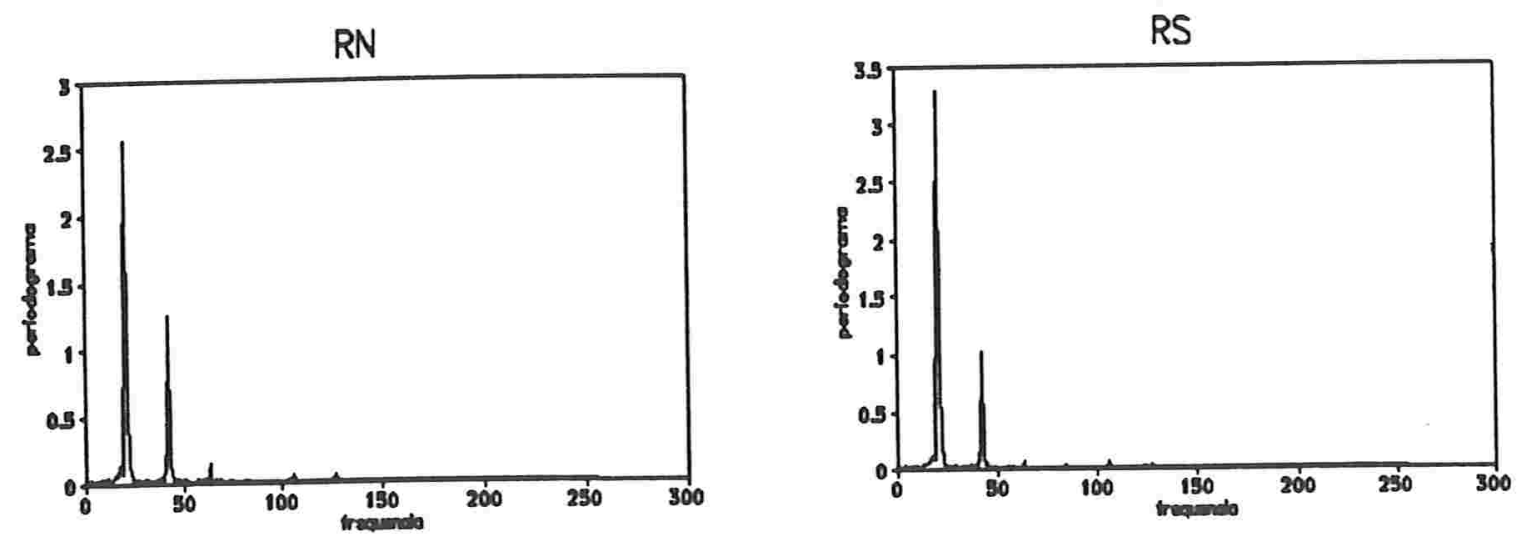

Figura 6.19. Periodograma médio de Fourier para os grupos RN e RS
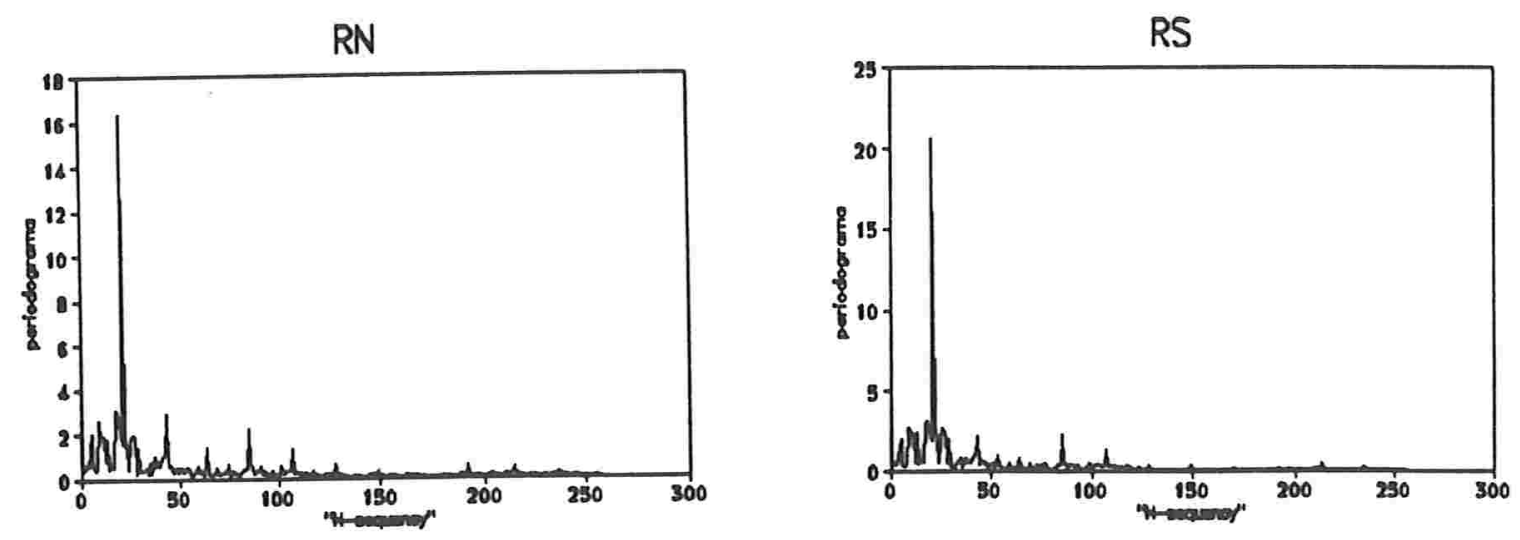

Figura 6.20. Periodograma médio de Walsh modificado para os grupos RN e RS 


\section{Referências Bibliográficas}

[1] Ahmed, N. and Rao, K.R. (1975), Orthogonal Transforms for Digital Signal Processing, New York: Springer-Verlag.

[2] Anderson, T.W. (1958), An Introduction to Multivariate Statistical Analysis, New York: Wiley.

[3] Beauchamp, K.G. (1975), Walsh Functions and Their Applications, London: Academic Press.

[4] Beauchamp, K.G. (1984), Applications of Waish and Related Functions, London: Academic Press.

[5] Bloomfield, P. (1976), Fourier Analysis of Time Series: An Introduction, New York: John Wiley.

* [6] Brillinger, D.R. (1969), A Search for a Relationship between Monthly Sunspot Numbers and Certain Climatic Series, Bull. Internat. Statist. Inst. 43, 293-306.

[7] Brillinger, D.R. (1973), The Analysis of Time Series Collected in an Experimental Design, in Multivariate Analysis III, ed. P.R. Krishnaiah, New York: Academic Press, pp. 241-256.

[8] Brillinger, D.R. (1980), Analysis of Variance Problems under Time Series Models, in Handbook of statistics (Vol. 1), ed. P.R.Krishnaiah, Amsterdam: North Holland, pp. 237-278.

[9] Brillinger, D.R. (1981), Time Series: Data Analysis and Theory (2nd ed.), San Francisco: Holden-Day.

[10] Brillinger, D.R. (1985), A Maximum Likelihood Approach to FrequencyWavenumber Analysis, IEEE Trans. on Acoustics, Speech and Signal Processing, ASSP, 33. 
[11] Fine, N. J. (1957), Fourier-Stieltjes Series of Walsh Functions, Transactions of the American Mathematical Society 86, 246-255.

[12] Goodman, N.R. (1963), Statistical Analysis Based on a Certain Multivariate Complex Gaussian Distribution, Ann. Math. Stat., 34.

[13] Green, B.F. and Tukey, J.W. (1960), Complex Analysis of Variance: General Problems, Psychometrika 25, 127-152.

[14] Hannan, E.J. (1970), Multiple Time Series, New York: John Wiley.

[15] Hannan, E.J. (1973), Central Limit Theorems for Time Series Regression, Z. Wahrschein. 26, 157-170.

[16] Harmuth, H.F. (1969a), Applications of Walsh Functions in Communications, IEEE Spectrum 6, 82-91.

[17] Harmuth, E.J. (1969b), Transmission of Information by Orthgonal Functions (2nd Ed. 1972), New York: Springer-Verlag.

[18] Harmuth, H.F. (1977), Sequency Theory: Foundations and Applications, New York: Academic Press.

[19] Iosifescu, M. and Theodorescu, R. (1969), Random Processes and Learning, Berlin: Springer.

[20] Kohn, R. (1980a), On the Spectral Decomposition of Stationary Time Series Using Walsh Functions I, Advances in Applied Probability 12, 183-199.

[21] Kohn, R. (1980b), On the Spectral Decomposition of Stationary Time Series Using Walsh Functions II, Advances in Applied Probability 12, 462-474.

[22] Krishnaiah, P.R. (1969), Simultaneous Test Procedures under General MANOVA Models, in Multivariate Analysis II, ed. P.R. Krishnaiah, New York: Academic Press, pp. 121-143.

[23] Leonov, V.P. and Shiryaev, A.N. (1960), Some Problems in the Spectral Theory of Higher Moments, II, Theory Prob. Appl.(USSR), Vol. 5. 
[24] Mardia, K.V., Kent, J.T. and Bibby, J.M. (1979), Multivariate Analysis, New York: Academic Press.

[25] Morettin, P.A. (1972), Walsh-Fourier Analysis of Time Series, Ph.D. dissertation, University of California, Berkeley, Dept. of Statistics.

[26] Morettin, P.A. (1974a), Limit Theorems for Stationary and DyadicStationary Processes, Bulletin of the Brazilian Mathematical Society 5 , 97-104.

[27] Morettin, P.A. (1974b), Walsh-Function Analysis of a Certain Class of Time Series, Stochastic Processes and Their Applications 4, 183-194.

[28] Morettin, P.A. (1976), Estimation of the Walsh spectrum, IEEE Trans. Inform. Theory 22, 106-107.

[29] Morettin, P.A. (1979), Análise Harmônica de Processos Estocásticos, $12^{0}$ Colóquio Brasileiro de Matemática. R.J: IMPA/CNPq.

[30] Morettin, P.A. (1981), Walsh Spectral Analysis, SIAM review 23, 279291.

[31] Morettin, P.A. (1983), A Note on a Central Limit Theorem for Stationary Processes, Journal of Time Series Analysis 4, 49-52.

[32] Morgenthaler, G.W. (1957), On Walsh-Fourier series, Trans. Am. Math. Soc. 84.

[33] Nagai, T. (1976), On Finite Walsh Transforms of a Dyadic Stationary Time Series, Res. Rep. Faculty Eng., Oita University.

[34] Philipp, W. (1969), The Central Limit Problem for Mixing Sequences of Random Variables, Z.Wahrschein. Verw. Gebiet. Vol. 12.

[35] Priestley, M.B. (1981), Spectral Analysis and Time Series (Vols, 1 and 2), New York: Academic Press.

[36] Rao, C.R. (1973), Linear Statistical Inference and Its Applications (2nd ed.), New York: John Wiley.

[37] Rosenblatt, M. (1956), A central Limit Theorem and a Strong Mixing Condition, Proc. Nat. Acad. Sci.(U.S.A.), Vol. 42. 
[38] Rosenblatt, M. (1961), Some Comments on Narrow Band-pass Filters, Quart. Appl. Math., Vol. 18.

[39] Roy, S.N., Gnanadesikan, R. and Srivastava, J.N. (1971), Analysis and Design of Certain Quantitative Multiresponse Experiments, Pergamon, Oxford.

[40] Scheffé, H. (1959), The Analysis of Variance, New York: John Wiley.

[41] Searle, S.R. (1971), Linear Models, New York: John Wiley.

[42] Shumway, R.H. (1970), Applied Regression and Analysis of Variance for Stationary Time Series, Journal of the American Statistical Association $65,1527-1546$.

[43] Shumway, R.H. (1988), Applied Statistical 'îme Series Analysis, Englewood Cliffs, NJ: Prentice-Hall.

[44] Skorokhod, A.V. (1956), Limit Theorems for Stochastic Processes, Theory Prob. Appl. 1, 261-290.

[45] Stoffer, D.S. (1985), Central Limit Theorems for Finite Walsh-Fourier Transforms of Weakly Stationary Time Series, Journal of Time Series Analysis 6, 261-267.

[46] Stoffer, D.S. (1987), Walsh-Fourier Analysis of Discrete Valued Time Series, Journal of Time Series Analysis 8, 449-467.

[47] Stoffer, D.S., Scher, M.S., Richarson, G.A., Day, N.L., and Coble, P.A. (1988), A Walsh-Fourier Analysis of the Effects of Moderate Maternal Alcohol Consumption on Neonatal Sleep-State Cycling, Journal of the American Statistical Association 83, 954-963.

[48] Stoffer, D.S. (1990), Multivariate Walsh-Fourier Analysis, Journal of Time Series Analysis 11, 57-73.

[49] Stoffer, D.S. (1991), Walsh-Fourier Analysis and Its Statistical Applications, Journal of the American Statistical Association 86, 461-485.

[50] Wahba, G. (1968), On the Distribution of Some Statistics Useful in the Analysis of Jointly Stationary Time Series, Ann. Math. Stat., 39. 
[51] Waldmeir, M. (1961), The sunspot activity in the years 1610-1960, Zurich: Schulthess. 


\section{Apêndice}

Apresentaremos, aqui, alguns programas que foram utilizados no Capítulo 6. Todos os programas apresentados neste apêndice foram desenvolvidos em QuickBASIC, versão 4.0 e os arquivos de entrada e de saída são da forma ASCII.

\section{Programa ANOVAF1.BAS}

O objetivo deste programa é calcular a estatística $F$ (4.18) para testar a hipótese

$$
H_{0}: \Omega(t)=0
$$

do modelo com um sinal comum,

$$
Y_{n}(t)=\mu_{n}+\Omega(t)+\epsilon_{n}(t)
$$

utilizando os coeficientes de Fourier da série com $\mathrm{T}$ pontos. $\mathrm{O}$ arquivo de entrada tem seguinte formato: (2n-1)-ésima coluna e (2n)-ésima coluna do arquivo correspondem aos coeficientes de Fourier da n-ésima série da amostra, onde $n=1, \cdots, N$ e $N$ é o número de séries da amostra. $\mathrm{O}$ arquivo de saída contem os valores da estatística. $\mathrm{F}$ em cada frequência. A listagem do programa é

CLS

INPUT "O nome do arquivo de entrada.........?: ", arq\$

INPUT "O número de séries a serem analisadas.?: ", amost\%

INPUT "O tamanho de cada série..............?: ", num\%

\{coloque $\frac{T}{2}$ pontos

INPUT "O nome do arquivo de saída............?: ", arqs\$

linha $\%=$ num $\%$ : coluna $\%=$ amost $\%+3$

DIM f(linha\%), $\mathrm{x}($ linha\%, coluna $\%), \mathrm{y}($ linha $\%$, coluna $\%)$

OPEN "I", \# 1, arq\$: OPEN "O", \# 2, arqs\$

\{leitura de dados\}

FOR lin $\%=1$ TO num $\%$ 
FOR col\%=1 TO amost $\%$

INPUT \# 1, $\mathrm{x}(\operatorname{lin} \%, \mathrm{col} \%)$ : INPUT \# 1, y(lin\%, col\%) NEXT

\section{NEXT}

FOR lin $\%=1$ TO num\%

FOR col\%=1 TO amost $\%$

$\mathrm{x}($ lin $\%$, amost $\%+1)=\mathrm{x}(\operatorname{lin} \%$, amost $\%+1)+\mathrm{x}(\operatorname{lin} \%, \operatorname{col} \%)$

$\mathrm{y}(\operatorname{lin} \%$, amost $\%+1)=y(\operatorname{lin} \%$, amost $\%+1)+y(\operatorname{lin} \%, \operatorname{col} \%)$

\{calcula soma\}

NEXT

$\mathrm{x}($ lin\%, amost $\%+1)=\mathrm{x}($ lin $\%$, amost $\%+1) /$ amost $\%$

$\mathrm{y}(\operatorname{lin} \%$, amost $\%+1)=\mathrm{y}(\operatorname{lin} \%$, amost $\%+1) / \operatorname{amost} \%$

\{calcula média\}

$\mathrm{x}($ lin $\%$, amost $\%+2)=\mathrm{x}($ lin $\%$, amost $\%+1) \wedge 2 \star$ amost $\%$

$\mathrm{y}($ lin $\%$, amost $\%+2)=y(\operatorname{lin} \%$, amost $\%+1) \wedge 2 \star$ amost $\%$

\{calcula soma dos quadrados\}

FOR col\%=1 TO amost\%

quadradol $=(x($ lin $\%$, col $\%)-x($ lin $\%$, amost $\%+1)) \wedge 2$

quadrado $2=(\mathrm{y}(\operatorname{lin} \%$, col\% $)-\mathrm{y}(\operatorname{lin} \%$, amost $\%+1)) \wedge 2$

$\mathrm{x}($ lin $\%$, amost $\%+3)=\mathrm{x}($ lin $\%$, amost $\%+3)+$ quadrado 1

$y($ lin $\%$, amost $\%+3)=y($ lin $\%$, amost $\%+3)+$ quadrado 2

NEXT

teste1 $=(\mathrm{x}(\operatorname{lin} \%$, amost $\%+2)+\mathrm{y}(\operatorname{lin} \%$, amost $\%+2)) *($ amost\%-1 $)$

teste $2=\mathrm{x}($ lin $\%$, amost $\%+3)+\mathrm{y}($ lin $\%$, amost $\%+3)$

testef $=$ testel $1 /$ teste 2

$\mathrm{f}(\mathrm{lin} \%)=$ testef

NEXT

FOR lin $\%=1$ to num\%

NEXT

PRINT \# 2, f(lin\%)

SYSTEM

\section{Programa ANOVAF2.BAS}

O objetivo deste programa é calcular a estatística $F$ para testar a interação entre os fatores

$H_{0}:(\Gamma \Delta)_{11}(t)=\cdots=(\Gamma \Delta)_{M 1}(t)=(\Gamma \Delta)_{12}(t)=\cdots=(\Gamma \Delta)_{M Q}(t)=0$, 
do modelo com dois fatores cruzados fixos, com $\mathrm{M}$ e $\mathrm{Q}$ níveis,

$$
Y_{l m q}(t)=\mu_{l m q}+\Omega(t)+\Gamma_{m}(t)+\Delta_{q}(t)+(\Gamma \Delta)_{m q}(t)+\epsilon_{l m q}(t)
$$

onde $l=1, \cdots, L, m=1, \cdots, M$ e $q=1, \cdots, Q$; utilizando os coeficientes de Fourier da série com $\mathrm{T}$ pontos. Os arquivos de entrada e de saída têm o mesmo formato que os do programa anterior. A listagem do programa é

\section{CLS}

INPUT "O numero de niveis do 1o. fator. ?: ", a\%

INPUT "O numero de niveis do 2o. fator......?: ", b\%

INPUT "O número de replicas em cada casela...?: ", r\%

INPUT "O tamanho de cada série. ?: ", T\%

\{coloque $\frac{T}{2}$ pontos

INPUT "O nome do arquivo de saída ?: ", arqs $\$$

DIM serier(a\%, b\%, r\%+1, T\%), seriei(a\%, b\%, r\%+1, T\%)

DIM yir(a\%, T\%), yii(a\%, T\%), yjr(b\%, T\%), yji(b\%, T\%)

DIM ygr(T\%), ygi(T\%), F(T\%), sqab(T\%), ssqr(T\%)

FOR i $\%=1$ TO a $\%$

FOR $j \%=1$ TO b\%

PRINT "O nome do arquivo que contem os dados para nivel"; i\%;

PRINT " do 1o. fator e nivel"; j\%; "do 2o. fator...?:";

INPUT" ", arq\$

CLOSE 1: OPEN "I", \# 1, arq\$

\{leitura de dados\}

FOR tt $\%=1$ TO T\%

FOR $k \%=1$ TO r $\%$

INPUT \# 1, serier(i\%, $\mathrm{j} \%, \mathrm{k} \%, \mathrm{tt} \%)$

INPUT \# 1, seriei(i\%, j\%, k\%, tt\%)

\section{NEXT}

NEXT

NEXT

NEXT

CLOSE: OPEN "O", \# 2, arqs\$

FOR $\mathrm{tt} \%=1$ TO T\%

somal $\mathrm{r}=0$ : soma $1 \mathrm{i}=0$

soma $2 \mathrm{r}=0$ : $\operatorname{soma} 2 \mathrm{i}=0$ 
soma $3 \mathrm{r}=0$ : soma $3 \mathrm{i}=0$

FOR $i \%=1$ TO a $\%$

soma $2 \mathrm{r}=0$ : soma $2 \mathrm{i}=0$

FOR $\mathrm{j} \%=1$ TO b\%

somal $\mathrm{r}=0$ : soma $1 \mathrm{i}=0$

FOR $\mathrm{k} \%=1$ TO $\mathrm{r} \%$

somal $\mathrm{r}=$ soma $1 \mathrm{r}+\operatorname{serier}(\mathrm{i} \%, \mathrm{j} \%, \mathrm{k} \%, \mathrm{tt} \%)$

somali=somali+seriei $(\mathrm{i} \%, \mathrm{j} \%, \mathrm{k} \%, \mathrm{tt} \%)$

soma $2 \mathrm{r}=\operatorname{soma} 2 \mathrm{r}+\operatorname{serier}(\mathrm{i} \%, \mathrm{j} \%, \mathrm{k} \%, \mathrm{tt} \%)$

soma $2 \mathrm{i}=$ soma $2 \mathrm{i}+\operatorname{seriei}(\mathrm{i} \%, \mathrm{j} \%, \mathrm{k} \%, \mathrm{tt} \%)$

soma $3 r=\operatorname{soma} 3 r+\operatorname{serier}(i \%, j \%, k \%$, tt $\%)$

soma3i $=$ soma3i+seriei $(\mathrm{i} \%, \mathrm{j} \%, \mathrm{k} \%, \mathrm{tt} \%)$

NEXT

serier $(\mathrm{i} \%, \mathrm{j} \%, \mathrm{r} \%+1, \mathrm{tt} \%)=$ soma $1 \mathrm{r} / \mathrm{r} \%$

seriei $(\mathrm{i} \%, \mathrm{j} \%, \mathrm{r} \%+1, \mathrm{tt} \%)=$ somali $/ \mathrm{r} \% \quad\left\{\right.$ calcule $\left.Y_{. m q}^{\wedge}(k)\right\}$

NEXT

$\operatorname{yir}(\mathrm{i} \%, \mathrm{tt} \%)=\operatorname{soma} 2 \mathrm{r} /(\mathrm{b} \% \star \mathrm{r} \%)$

yii $(\mathrm{i} \%, \mathrm{tt} \%)=$ soma $2 \mathrm{i} /(\mathrm{b} \% \star \mathrm{r} \%) \quad\left\{\right.$ calcule $\left.Y_{. m}{ }^{\wedge} .(k)\right\}$

NEXT

$\operatorname{ygr}(\mathrm{tt} \%)=\operatorname{soma} 3 \mathrm{r} /(\mathrm{b} \% \star \mathrm{a} \% \star \mathrm{r} \%)$

$\operatorname{ygi}(\mathrm{tt} \%)=$ soma3i $/(\mathrm{b} \% \star \mathrm{a} \% \star \mathrm{r} \%) \quad\{$ calcule $Y \wedge(k)\}$

NEXT

FOR tt $\%=1$ TO T $\%$

FOR $\mathrm{j} \%=1$ TO $\mathrm{b} \%$

soma $4 \mathrm{r}=0$ : soma $4 \mathrm{i}=0$

FOR i $\%=1$ TO a $\%$

FOR $\mathrm{k} \%=1$ TO $\mathrm{r} \%$

soma $4 \mathrm{r}=$ soma $4 \mathrm{r}+\operatorname{serier}(\mathrm{i} \%, \mathrm{j} \%, \mathrm{k} \%, \mathrm{tt} \%)$

soma $4 \mathrm{i}=$ soma $4 \mathrm{i}+\operatorname{seriei}(\mathrm{i} \%, \mathrm{j} \%, \mathrm{k} \%, \mathrm{tt} \%)$

NEXT

NEXT

$\mathrm{yjr}(\mathrm{j} \%, \mathrm{tt} \%)=\operatorname{soma} 4 \mathrm{r} /(\mathrm{a} \% \star \mathrm{r} \%)$

yji $(\mathrm{j} \%, \mathrm{tt} \%)=$ soma $4 \mathrm{i} /(\mathrm{a} \% \star \mathrm{r} \%) \quad\left\{\right.$ calcule $\left.Y_{. . q}^{\wedge}(k)\right\}$

NEXT

NEXT

FOR tt $\%=1$ TO T\%

somal $\mathrm{r}=0$ : somal $\mathrm{i}=0$ 


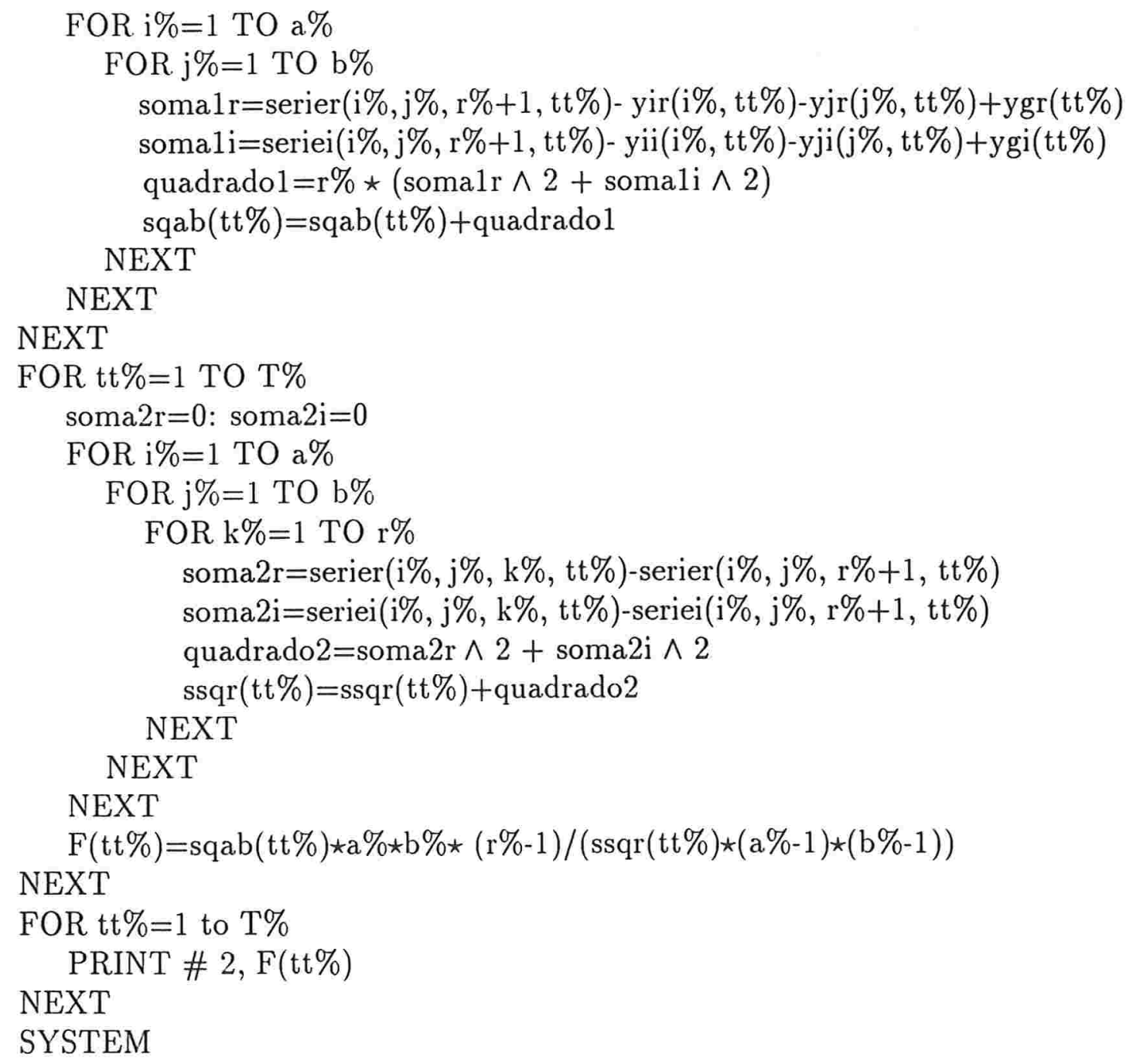

III. Programa ANOVAW1.BAS

Este programa calcula a estatística (5.14) para testar a hipótese

$$
H_{0}: \Omega(t)=0
$$

do modelo com um sinal comum,

$$
X_{n}(t)=\Theta_{n}+\Omega(t)+\epsilon_{n}(t)
$$


utilizando a transformada de Walsh. Ele contem a subrotina FWT que executa o algoritmo FWT (transformada rápida de Walsh) para obter as ordenadas de Walsh de uma série, cujo tamanho é da forma $2^{p}$. $\mathrm{O}$ arquivo de entrada é da seguinte forma: n-ésima coluna do arquivo corresponde aos dados originais da n-ésima série, onde $n=1, \cdots, N$ e $N$ é o número de séries da amostra. $\mathrm{O}$ arquivo de saída contem os valores da estatística $\mathrm{F}$ em cada sequência. A listagem do programa é

\section{CLS}

ii $\%=2$

INPUT "O nome do arquivo de entrada ?: ", arqe $\$$

INPUT "O numero de series ?.......... ", amost\%

INPUT "O tamanho de cada serie.......: ", num\%

INPUT "O nome do arquivo de saida ?..: ", arqs\$

linha $\%=$ num $\%$ : coluna $\%=$ amost $\%+4$

DIM ipower\%(150), x(linha\%, coluna\%), y(linha\%, coluna\%)

OPEN "I", \# 1, arqe\$: OPEN "O", \# 2, arqs\$

FOR lin $\%=1$ TO num $\% 178$

FOR col $\%=1$ TO amost $\%$

INPUT \# 1, x(lin\%, col\%)

NEXT

NEXT

FOR col $\%=1$ TO amost $\%$

GOSUB FWT

\section{NEXT}

FOR lin $\%=1$ TO num $\%$

FOR $\operatorname{col} \%=1$ TO amost $\%$

$\mathrm{x}(\operatorname{lin} \%, \operatorname{amost} \%+1)=\mathrm{x}(\operatorname{lin} \%, \operatorname{amost} \%+1)+\mathrm{x}(\operatorname{lin} \%, \operatorname{col} \%)$

NEXT $\{$ calcula soma $\}$

$\mathrm{x}(\operatorname{lin} \%$, amost $\%+1)=\mathrm{x}(\operatorname{lin} \%$, amost $\%+1) / \operatorname{amost} \%$

$\mathrm{x}($ lin $\%$, amost $\%+2)=\mathrm{x}(\operatorname{lin} \%$, amost $\%+1) \wedge 2 \star$ amost $\%$

FOR col $\%=1$ TO amost $\%$

quadrado $=(x($ lin $\%$, col $\%)-x($ lin $\%$, amost $\%+1)) \wedge 2$

$\mathrm{x}(\operatorname{lin} \%$, amost $\%+3)=\mathrm{x}(\operatorname{lin} \%$, amost $\%+3)+$ quadrado

\section{NEXT}

testef $=x($ lin $\%$, amost $\%+2) \star($ amost $\%-1) / x(\operatorname{lin} \%$, amost $\%+3)$

$\mathrm{x}(\operatorname{lin} \%$, amost $\%+4)=$ testef 
NEXT

FOR lin $\%=1$ TO num $\%$

PRINT \# 2, $\mathrm{x}(\operatorname{lin} \%$, amost\%+4)

NEXT

SYSTEM

FWT:

FOR i $\%=1$ TO num $\%$

$\mathrm{ib} \%=\mathrm{i} \%-1$

$\mathrm{il} \%=1$

9:

$$
\begin{aligned}
& \text { ibd } \%=\text { INT(ib } \% / 2) \\
& \text { ipower } \%(i 1 \%)=1 \\
& \text { IF ib } \%=i b d \% \star 2 \text { THEN ipower } \%(i l \%)=0 \\
& \text { IF ibd } \%=0 \text { THEN GOTO } 10 \\
& \text { ib } \%=i b d \% \\
& \text { il } \%=i l \%+1 \\
& \text { GOTO } 9
\end{aligned}
$$

10:

$$
\begin{aligned}
& \text { ip } \%=1 \\
& \text { ifac } \%=\text { num } \% \\
& \text { FOR } i 1 \%=1 \text { TO il } \%
\end{aligned}
$$$$
\text { ifac } \%=\operatorname{INT}(\text { ifac } \% / 2)
$$

$$
\begin{aligned}
& \text { ip } \%=\text { ip } \%+\text { ifac\% } \% \text { ipower } \%(i 1 \%) \\
& \text { NEXT }
\end{aligned}
$$$$
y(i p \%, \operatorname{col} \%)=x(i \%, \operatorname{col} \%)
$$

NEXT

FOR i $\%=1$ TO num $\%$

$$
\mathrm{x}(\mathrm{i} \%, \operatorname{col} \%)=\mathrm{y}(\mathrm{i} \%, \operatorname{col} \%)
$$

NEXT

iter $\%=0$

irem $\%=$ num $\%$

1:

irem $\%=\operatorname{INT}($ irem $\% / 2)$

IF irem $\%=0$ THEN GOTO 2

iter $\%=$ iter $\%+1$

GOTO 1 
2:

FOR $m \%=1$ to iter $\%$

IF $\mathrm{m} \%=1$ THEN nump $\%=1$

ELSE nump $\%=$ nump $\% \star 2$

END IF

mnum $\%=\operatorname{INT}($ num $\% /$ nump $\%)$

mnum $2 \%=\operatorname{INT}(\operatorname{mnum} \% / 2)$

alph $=1$

FOR mp $\%=1$ TO numo $\%$

$\mathrm{ib} \%=(\mathrm{mp} \%-1) \star \mathrm{mnum} \%$

FOR mp2\%=1 TO mnum $2 \%$

mnum $21 \%=$ mnum $2 \%+\mathrm{mp} 2 \%+\mathrm{ib} \%$

$\mathrm{iba} \%=\mathrm{ib} \%+\mathrm{mp} 2 \%$

$\mathrm{y}(\mathrm{iba} \%, \operatorname{col} \%)=\mathrm{x}(\mathrm{iba} \%, \operatorname{col} \%)+\mathrm{alph} \star \mathrm{x}(\operatorname{mnum} 21 \%, \operatorname{col} \%)$

$y(\operatorname{mnum} 21 \%, \operatorname{col} \%)=x(\mathrm{iba} \%, \operatorname{col} \%)-$ alph $\star x(\operatorname{mnum} 21 \%, \operatorname{col} \%)$

NEXT

IF ii $\%=2$ THEN alph=-alph

NEXT

FOR i $\%=1$ TO num $\%$

$x(i \%, \operatorname{col} \%)=y(i \%, \operatorname{col} \%)$

NEXT

NEXT

$\mathrm{r}=1 / \mathrm{SQR}$ (num\%)

FOR i $\%=1$ TO num $\%$

$\mathrm{x}(\mathrm{i} \%, \operatorname{col} \%)=\mathrm{x}(\mathrm{i} \%, \operatorname{col} \%) \star \mathrm{r}$

NEXT

RETURN

\section{Programa ANOVAW2.BAS}

Este programa calcula a estatística (5.28) para testar a hipótese

$$
H_{0}: \alpha_{1}(t)=\cdots=\alpha_{M}(t)=0
$$

do modelo com um fator,

$$
X_{l m}(t)=\Theta_{l m}+\mu(t)+\alpha_{m}(t)+\epsilon_{l m}(t)
$$


utilizando a transformada de Walsh. Ele também contem a subrotina FWT. Os arquivos de entrada e de saída têm o mesmo formato que os do programa anterior. A listagem do programa é

\section{CLS}

ii $\%=2$

INPU "O numero de grupos (valor de M)..? ",ng\%

DIM $\operatorname{arq} \$(n g \%)$

FOR a $\%=1$ TO ng\%

INPUT "O nome do arquivo de entrada(";a\%;"). ?: ", arq\$(a\%)

\section{NEXT}

INPUT "O numero de series ?..........: ", amost\%

INPUT "O tamanho de cada serie........ ", num\%

INPUT "O nome do arquivo de saida ?... ", arqs\$

linha $\%=$ num $\%$ : coluna $\%=$ amost $\%+1$

DIM ipower\%(150), x(linha\%, coluna\%, ng\%), y(linha\%, coluna\%, ng\%)

DIM F(linha\%, 4)

FOR a $\%=1$ TO ng\%

CLOSE: OPEN "I", \# 1, arqe\$(a\%)

FOR lin $\%=1$ TO num $\%$

FOR $\operatorname{col} \%=1$ TO amost $\%$

INPUT \# 1, x(lin\%, col\%, a\%)

NEXT

NEXT

NEXT

OPEN "O", \# 2, arqs\$

FOR a $\%=1$ TO ng\%

FOR $\operatorname{col} \%=1$ TO amost $\%$

GOSUB FWT

NEXT

\section{NEXT}

FOR a $\%=1$ TO ng\%

FOR lin $\%=1$ TO num $\%$

FOR col $\%=1$ TO amost $\%$ $x(\operatorname{lin} \%$, coluna $\%, a \%)=x(\operatorname{lin} \%$, coluna $\%, a \%)+x(\operatorname{lin} \%, \operatorname{col} \%, a \%)$ NEXT \{ calcula soma $\}$

$\mathrm{x}(\operatorname{lin} \%$, coluna\%, $\mathrm{a} \%)=\mathrm{x}(\operatorname{lin} \%$, coluna $\%, \mathrm{a} \%) / \mathrm{amost} \%$ 
NEXT

NEXT

FOR lin $\%=1$ TO num $\%$

FOR a $\%=1$ TO ng\%

$\mathrm{F}(\operatorname{lin} \%, 1)=\mathrm{F}(\operatorname{lin} \%, 1)+\mathrm{x}(\operatorname{lin} \%$, coluna $\%, \mathrm{a} \%)$

NEXT

$\mathrm{F}(\operatorname{lin} \%, 1)=\mathrm{F}(\operatorname{lin} \%, 1) / \mathrm{ng} \%$

FOR a $\%=1$ TO ng $\%$

$\mathrm{F}(\operatorname{lin} \%, 2)=\mathrm{F}(\operatorname{lin} \%, 2)+(\mathrm{x}(\operatorname{lin} \%$, coluna $\%, \mathrm{a} \%)-\mathrm{F}(\operatorname{lin} \%, 1)) \wedge 2$

NEXT

$\mathrm{F}(\operatorname{lin} \%, 2)=\operatorname{amost} \% \star \mathrm{F}(\operatorname{lin} \%, 2)$

FOR a $\%=1$ TO ng\%

FOR col $\%=1$ TO amost $\%$

quadrado $=(x(\operatorname{lin} \%$, col $\%, a \%)-x(\operatorname{lin} \%$, coluna $\%, a \%)) \wedge 2$

$\mathrm{F}(\operatorname{lin} \%, 3)=\mathrm{F}(\operatorname{lin} \%, 3)+$ quadrado

\section{NEXT}

NEXT

testef $=(F(\operatorname{lin} \%, 2) \star n g \% *(\operatorname{amost} \%-1)) / F((\operatorname{lin} \%, 3) \star(n g \%-1))$

NEXT

$\mathrm{F}(\operatorname{lin} \%, 4)=$ testef

FOR lin $\%=1$ TO num $\%$

PRINT \# 2, F(lin\%, 4)

NEXT

SYSTEM

FWT:

FOR i $\%=1$ TO num $\%$

$\mathrm{ib} \%=\mathrm{i} \%-1$

$\mathrm{i} 1 \%=1$

9:

$\mathrm{ibd} \%=\mathrm{INT}(\mathrm{ib} \% / 2)$

ipower\%(il\%) $=1$

IF ib\%=ibd $\%$ * 2 THEN ipower\%(il\%)=0

IF ibd $\%=0$ THEN GOTO 10

$\mathrm{ib} \%=\mathrm{ibd} \%$

$\mathrm{i} 1 \%=\mathrm{i} 1 \%+1$

GOTO 9 
10:

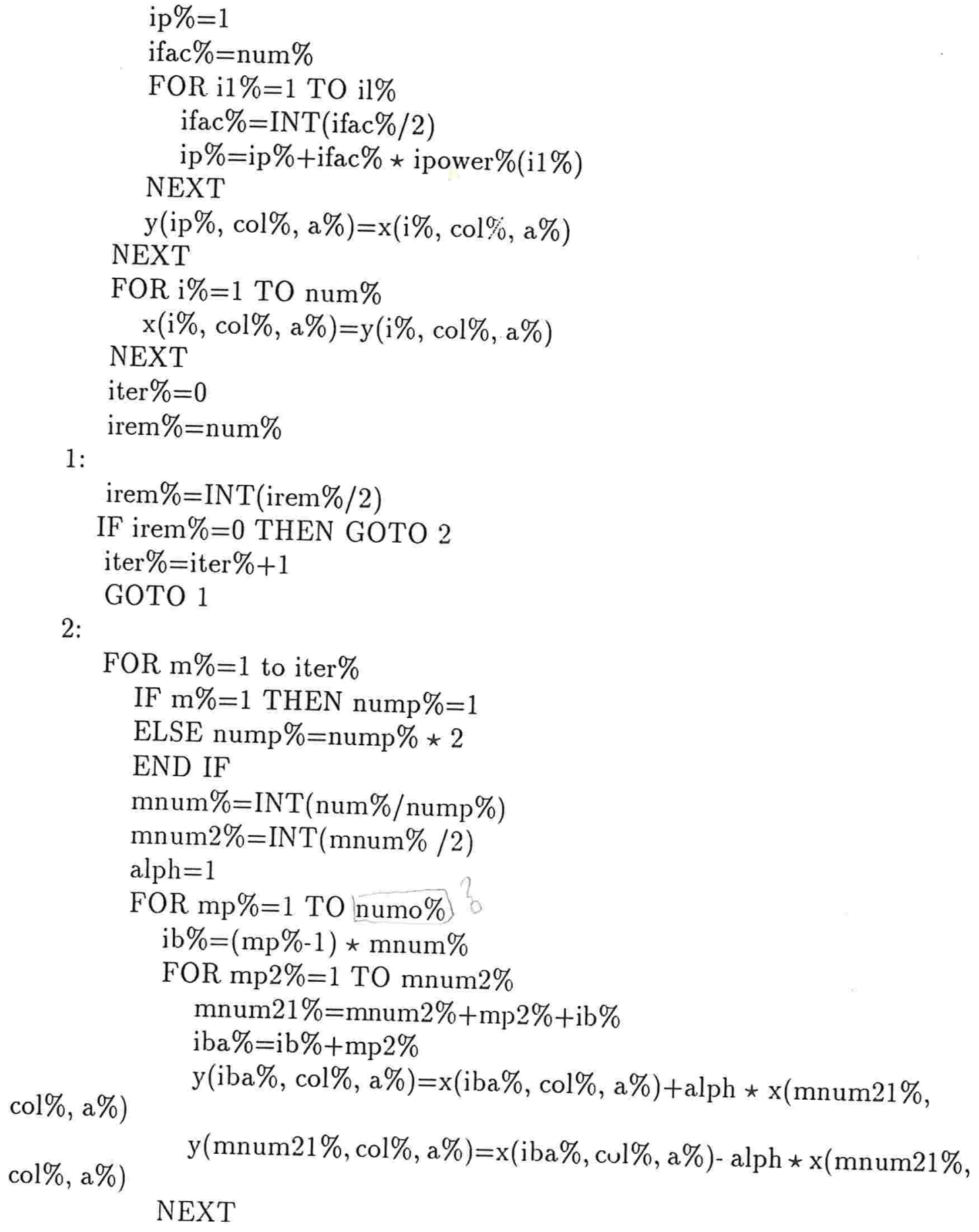


IF ii $\%>=2$ THEN alph=-alph

NEXT

FOR i $\%=1$ TO num $\%$

$\mathrm{x}(\mathrm{i} \%, \operatorname{col} \%, \mathrm{a} \%)=\mathrm{y}(\mathrm{i} \%, \mathrm{col} \%, \mathrm{a} \%)$

NEXT

NEXT

$\mathrm{r}=1 / \mathrm{SQR}($ num $\%)$

FOR i $\%=1$ TO num $\%$

$x(i \%, \operatorname{col} \%, a \%)=x(i \%, c o l \%, a \%) \star r$

NEXT

RETURN 